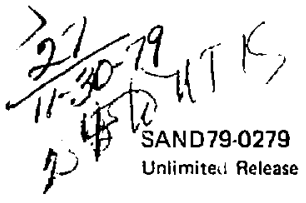
\title{
MASTER
}

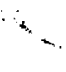

\section{Basic Data Report for Drillhole WIPP 25 (Waste Isolation Pilot Plant - WIPP)}

Sandia Laboratories and

United States Geological Survey 
TABLE OF OONTENTS

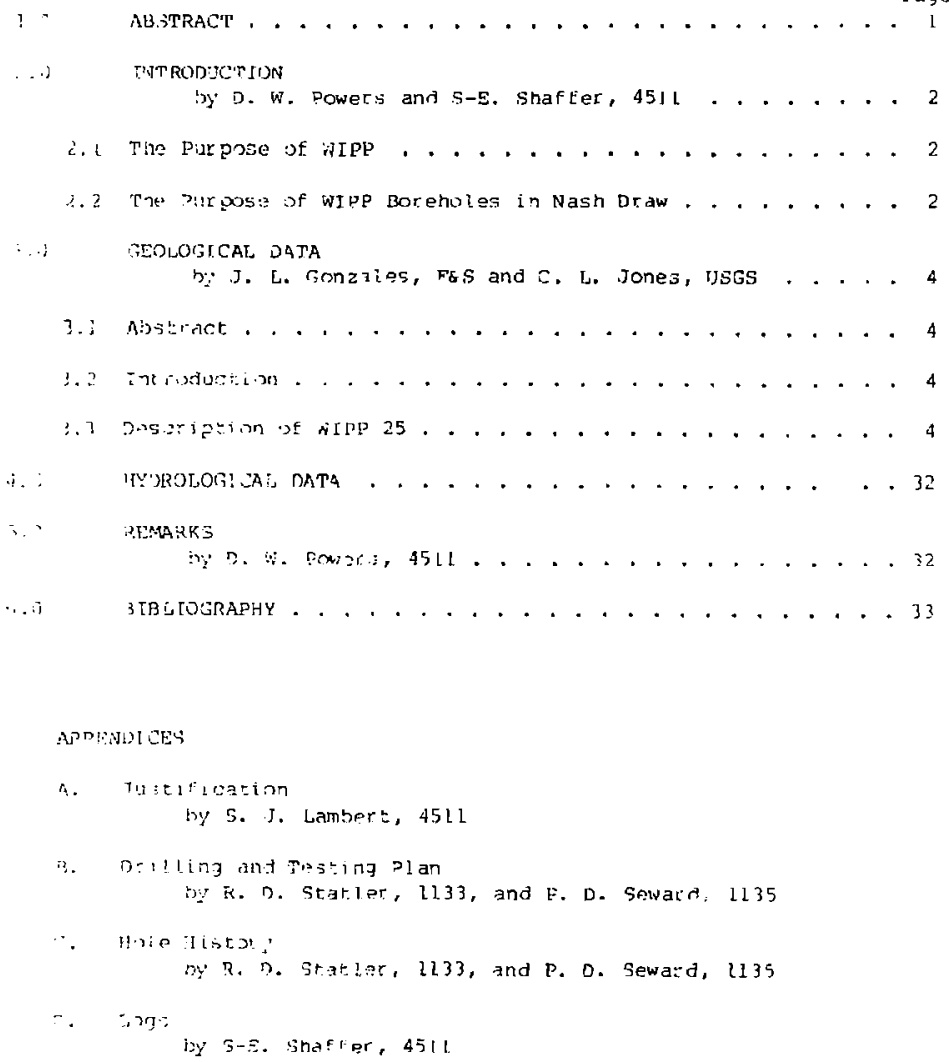




\subsection{ABSTRACT}

WIPP 25 was drilled on the eastern edge of Nash Draw (SW 1/4, Sec. 15, T225, R30E) in Eddy County, New Mexico, to determine subsurface itratigraphy and examine dissolution features above undisturbed salt in the salado Fomation, Determination of dissolution rates will refine previous estimates and provide short-term (geologically) rates for wipp -irik assessments. The borehole encountered, from top to bottom, Pl zist:ocene sediments 117' with fill material for pad), Dewey Lake Red Beds (215), Rustler Formation (333\%), and 90' of the upper salado Formation. A dissolution residue, 37' thick, is at the top of the salado formation overlying halite-r:ch beds. In addition to obtaining nearly cont i nuous core from the surface to total depth (655)), geophysical logs wers taken to measure acoustic velocities, density, radioactivity, and sornation resistivity. An interpretive report on dissolutici in Nash orav ill be based on combined borehole basin data, surface mappint, and laboratory analyses of Nash Draw rocks and Fluids. The NIPP is to demonstrate (through limited operations) disposal tachnology for transuranic defense wastes and to then be converted to a repository. The WIPP will also provide research facilities for intoractions between high-level waste and salt. 


\subsection{INTRODUCTION}

by

D. W. Powers and S-E. Shaffer ${ }^{1}$

Tha introduction describes background information on the waste $1=:^{\prime}$, in Piist Plant (WIPP) and the investigations in Nash Draw which $s=1$ ute NIP? 25.

\section{$\therefore$ THe 7:16 pos:? OE WIPP}

Tile pur Doss. af the WIPP is distinct from that of several other A d ats for the jksposal of radioactive waste. The WIPP is planned to a $\therefore x=7: 7 \exists t j o \gamma^{\prime} \mathrm{s}$ detense programs of over 30 years. After a period (j-I) yar;j of limited (pilot) operation it is anticipated that the wIPp :1: : ;onverser to a full-scale repository for permanent disposal of

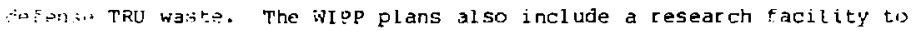

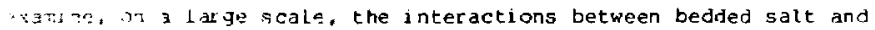
1:-12y: rad:oact) ve wasto resulting from thermal and radiation : ista. A pepartenent af Ener $y_{Y}$ (DOE) Tagk force has recominended that

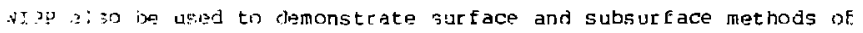
anding, stoming and jisposing of up to 1,000 canisters of spent reactor

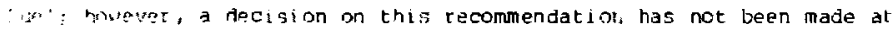
thi: tine. DCE ha: axpressed an intent to request licensing of the WIPP

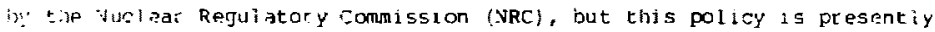
-later 1.jousion between the DOF and congzess.

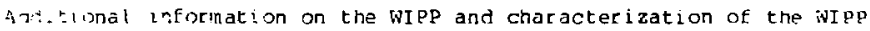
ite $\pi: \pi$, he enand in powers, et. al. (1978).

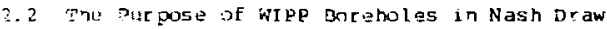

Tho seigin of Nush Draw, located several miles west of the NIpp site, ?ci:; mos: rommonly beer attributed to solution of underlying soluble "ypar: to cooss and subsidence of the overlying racks le.g*, Vine, 1963; ronis, 1973). The concern with the development of Nash Draw is to fatermine dissolution rates and/or processes such that the long-term 
threat to the WIPP may be evaluated. Bachman and Johnson (1973) estimated the lateral movement of the salt diseolution front as 6 to 8 miles per million years bince the end of Ogallala time. Bacnan (1974) also estimated the average rate of vertical dissolution in the area of Nash Draw over the past 600,000 years; that rate 19 about 330 feet p: aillion years. Piper (1973) estimated the vertical dissolution rate as sbout 160 feet per million years; Swerson (in Bachman and Johnson, 1973) independently estimated the rate of vertical dissolution as about 500 zet per million years. Bachman (1976) recognlzes additional, older episodes of dissolution in southeastern New mexico which imply that the aver age rates may be too high over the shorter ime period. The estimates of dissolution as average rates imply little appsent long-term threat to the WIPP.

The important problem remaining then is to determine the short-term (geologically) rates to assess any threat to the WIPP. The dissolution which occurred may be episodic rather than continuous. The Nash Draw boreholes (WIPP 25 through 30 ) are a direct approach to determining the processes and rates over shorter times. The processes can be determined by direct analysis of the core and borehole information. Stratigraphic analyses can reveal the affected beds and general time frame. Mineralogical and geochemical analyses of dissolution products may indicate process and ages. Hyydrologic analysis is to determine if dissolution is currently active. The analysis of data $f r c m$ all boreholes may be combined with other data sources to interpret these processes and rates.

This is a report of basic geological and hydrological data from WIPP 25 as a pat of the investigation of disgolution in Nash Draw.

Additional details regarding the background and justification for WIPP 25 are found in Appendix A. 


\title{
3. 0 GEOLOGICAL DATA
}

by

\author{
J. L. Gonzales ${ }^{1}$ and C. L. Jones ${ }^{2}$
}

\subsection{Abstract}

Borehole wrpp 25 was drjlled in east-central Eddy County, New Mexiso, in August and septenber, 1978. It penetrated unnamed deposits of Pleistocene age and the Dewey Lake Red Beds, the Rustler Formation and ivat of the Salado Formation, atl of Permian age. Detailed lithologic and geopiysizal logs of the borehole are presented in this chapte:.

2. ? Ineristuction

Borehula kIPP 2515 one nt a series of exploratory boreholes drilled

7. (i) detertine the stritigraphy of near-surface formations, (2) sxanl he the relationship hat ween subsurface struture and surficial Eeatures and deposits, and (3) investigate the atssolution of soluble tocks (: ock salt, gyosum, and dolomite) by groundwater.

Ihe axploratory arilling was done on behal of the WIPP (Waste 15rlazion Pist Plant! Project Dffice of the DOE fU.S. Department of Snergit:

\section{3 Descr 1 Fta on of WIPP 25}

wIPP 25 is Located in central eastern Eddy County, MM, in the

Sir if Sez. 15, T22S, R3uE (Figure 1). The borehole was drilled durjng Augut: and september, 1973, to a depth of 655 feet, measured from a Jand surface altiturle of 3,213 feet above MSL (mean sea level). Consecutive wites were taken fom the surkace to a deptin of 650.3 feet. The cores wort? axamined and logyed at the driul site, and a detalled lithologic log s: the borehole was preparad (Figure 2; Table 3) by S. L. Drellack, Ir., 1. t. Fonzales, and A. F. MeIntyre of Fos (Fenix \& Scisson, Inc.). The ahe i jget ixorehole history is given in Table 1, znd the stratigraphis Eutrear; of the hole in Table 2 .

IFenix \& Scisson, Inc., Carlsbad, NM.

?u.s. Genlogical survey, Denver, co. 


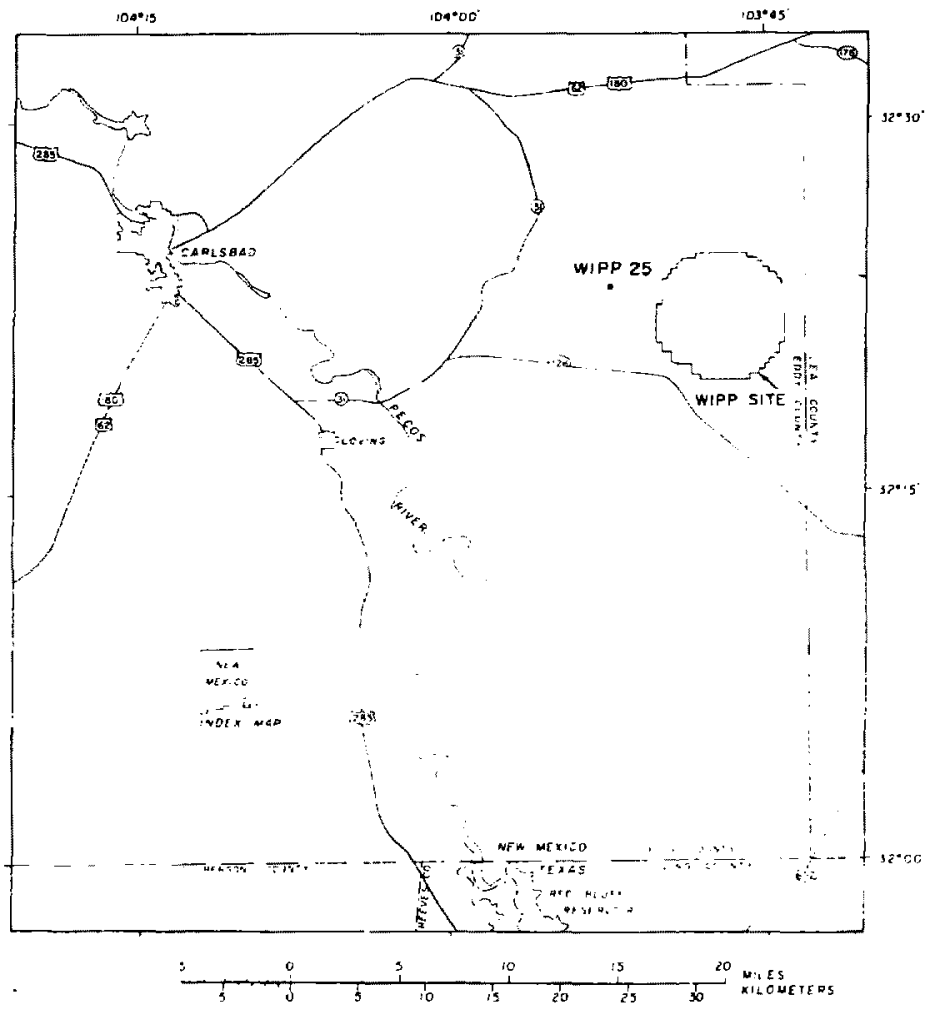

riqure 1. -index man showing lacation of borencle WIPP-25. 
SALADO FORMATION IUPPER PART
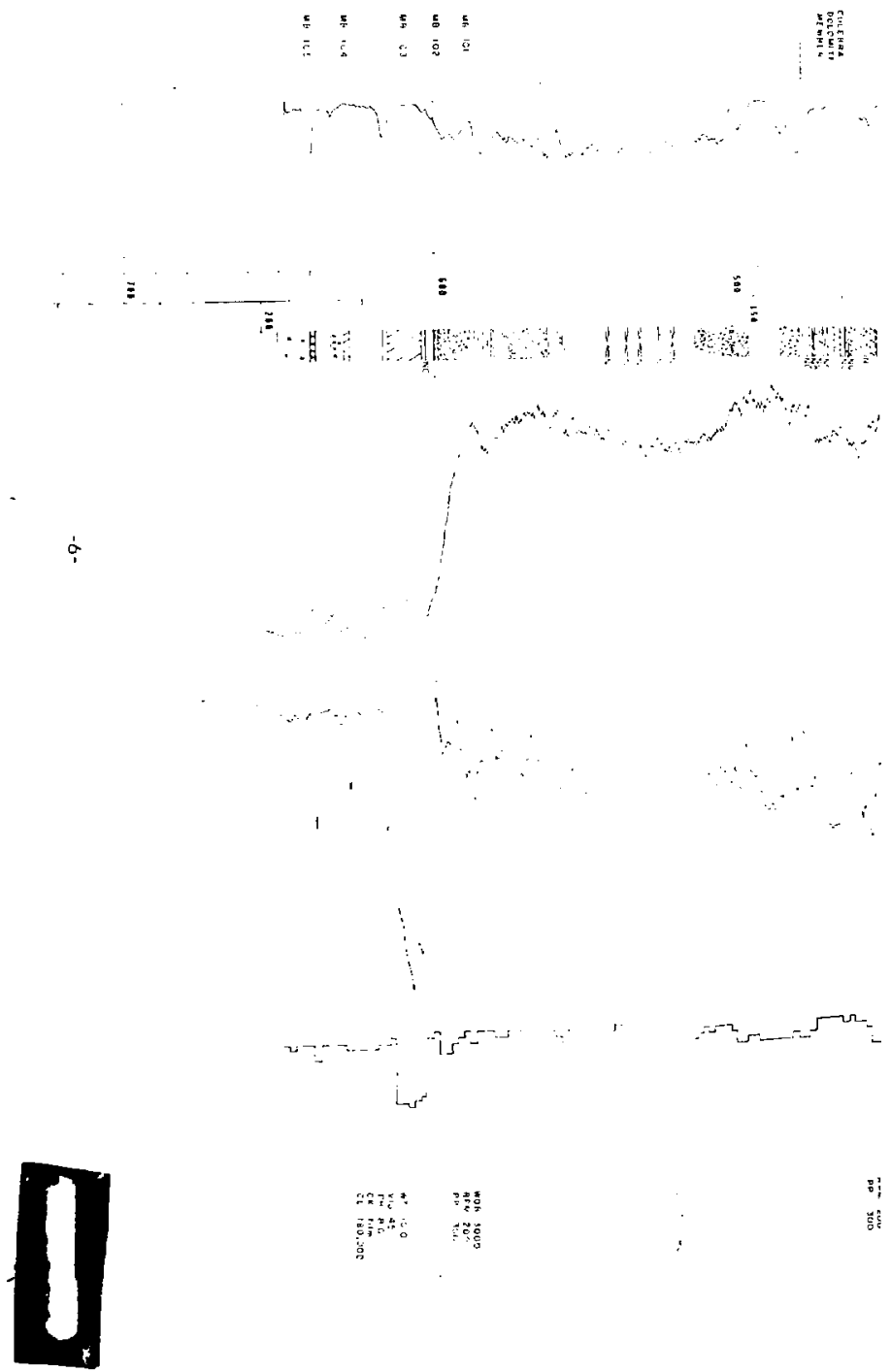

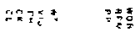

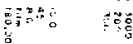

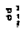

g. 


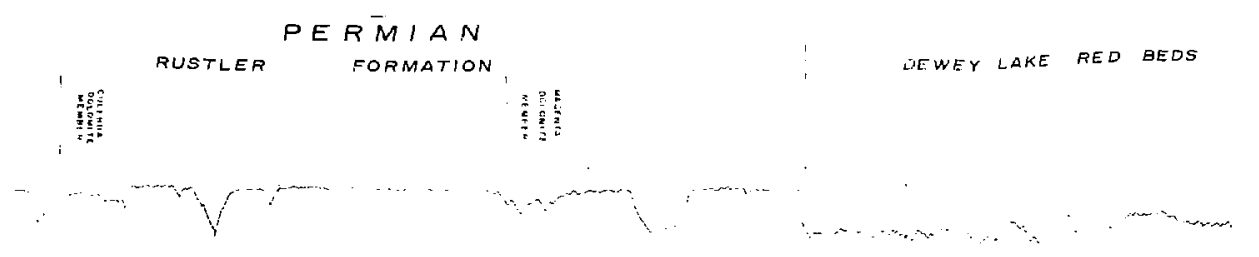

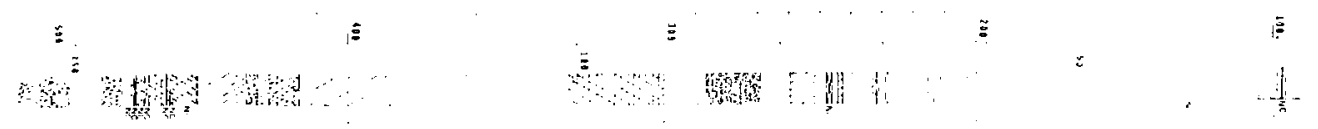

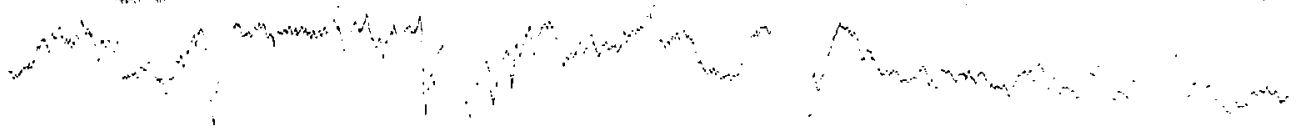

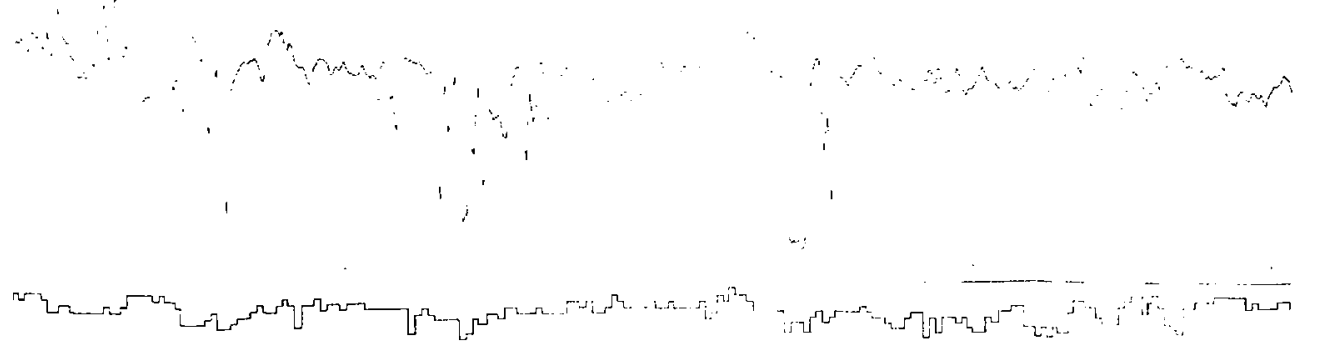

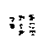

วิดัดั

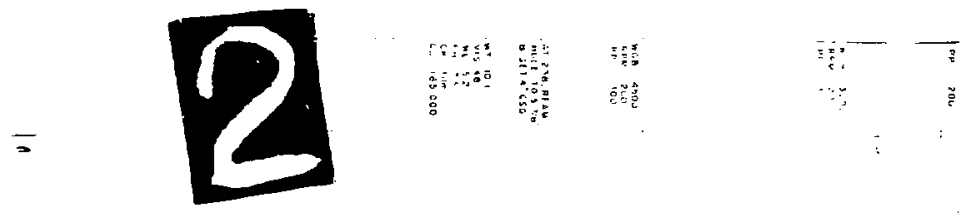


EY LAKE RED BEDS

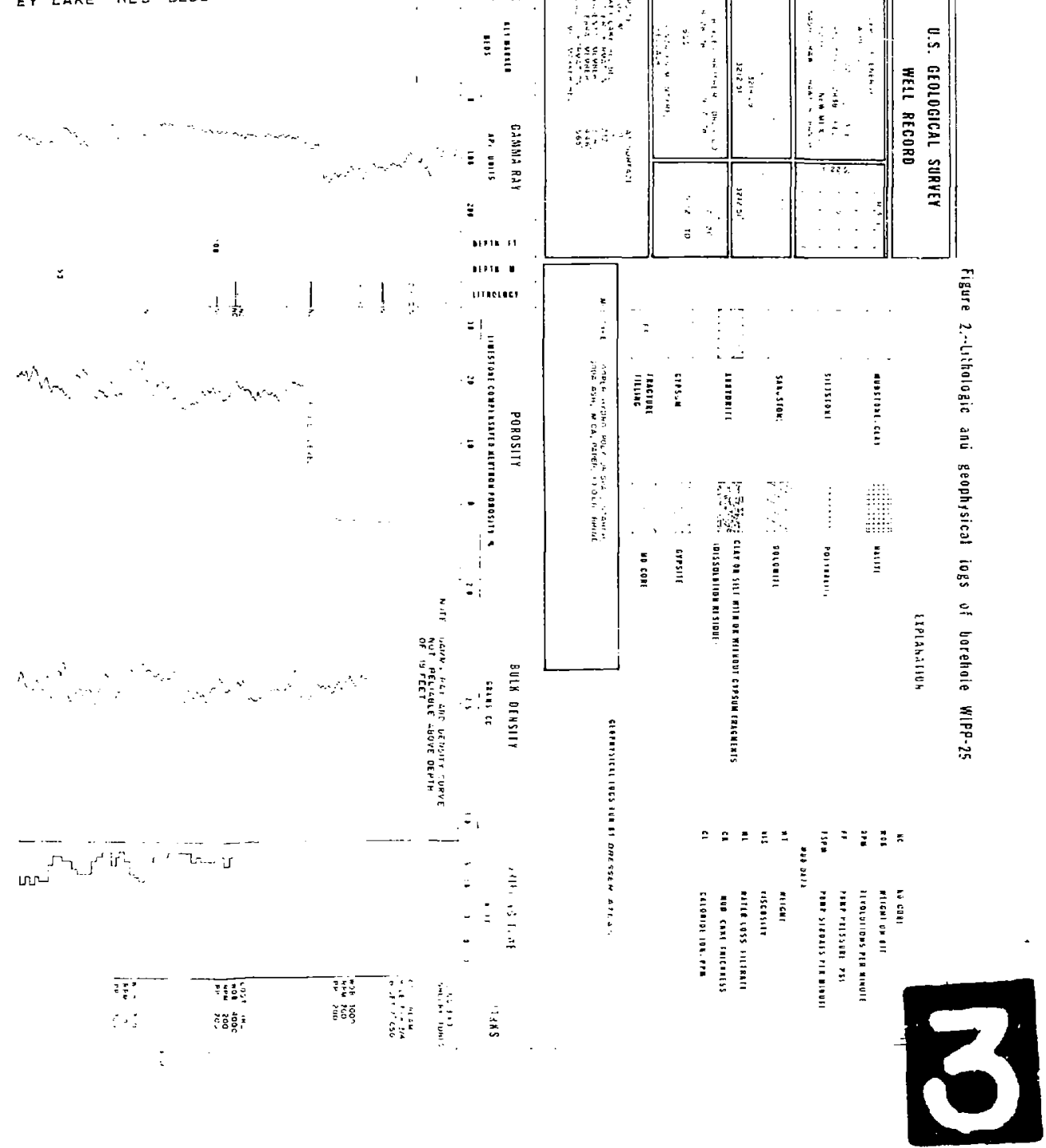


Table l.--Abr; dged hiseory of borehole Hrpp-2s

Loctiolot: Sec, is, T, 22 S., R. $30 \mathrm{E}$.

$1,852.32$ feet from south line

$2,838.1$ feet from east line

ALiITULE (LAND SURFACE): 3,212.51 feet. Datum for depth measurements in dritling and logging operations.

LITHOLOGIL LOG PREPARED BY: S. L. Orellack, Jr, , J, L, Gonzales, and A. F. McIntyre

JR!:LING CCNTRACTOR: Boyles Bros, Jrilling Co,

FETLLING RECORD: Commenced drilling on August 28, 1978, and completed on September 7. 1978, at 655 feet below land surface.

Lost circulation between depths of 78 and $88 ; 90$ and 108 ; and 522 and 526 feet.

After completion of coring, hole opened from $57 / 8$ to $77 / 3$ inches arid 648 feet of $51 / 2$-inch casing set and cemented to surface.

Hole ready for conversion to hydrologic observation well.

\begin{tabular}{|c|c|c|c|c|c|c|c|}
\hline $\begin{array}{c}\text { Core } \\
\text { No. }\end{array}$ & $\begin{array}{l}\text { Oepth } \\
\text { interval } \\
\text { in feet }\end{array}$ & RPM & $\begin{array}{l}\text { Weight } \\
\text { on bit } \\
\text { (ibs) }\end{array}$ & $\begin{array}{l}\text { Circulating } \\
\text { pressure } \\
\left(1 \mathrm{~b} / \mathrm{in}^{2}\right)\end{array}$ & $\begin{array}{l}\text { Feet } \\
\text { cored }\end{array}$ & $\frac{\text { Feet }}{\text { recovered }}$ & $\begin{array}{l}\text { Percent } \\
\text { recovered }\end{array}$ \\
\hline $\begin{array}{r}1 \\
2 \\
3 \\
4 \\
5 \\
6 \\
7 \\
8 \\
9 \\
10 \\
11 \\
12 \\
13 \\
14 \\
15 \\
16 \\
17 \\
18 \\
19 \\
20 \\
21 \\
22 \\
23 \\
24 \\
25 \\
26 \\
27 \\
28 \\
29 \\
30 \\
31 \\
32 \\
33 \\
34 \\
35 \\
36 \\
37 \\
38 \\
39 \\
40 \\
41 \\
42\end{array}$ & $\begin{array}{r}0=1.5 \\
1.5-3.0 \\
1.0-5.0 \\
6.0-9.0 \\
9.0-12.0 \\
12.0-15.0 \\
15.0-18.0 \\
18.0-21.0 \\
21.0-26.0 \\
26.0-32.0 \\
32.0-36.0 \\
36.8-41.0 \\
41.0-50.0 \\
50.0-60.0 \\
60.0-70.0 \\
70.0-78.0 \\
78.0-88.0 \\
88.0-90.0 \\
90.0-98.0 \\
98.0-108.0 \\
108.0-118.0 \\
118.0-128.0 \\
128.0-130.5 \\
130.5-140.0 \\
140.0-150.0 \\
150.0-1 \\
160.0-168.0 \\
168.0-178.0 \\
178.0-189.0 \\
188.0-198.0 \\
198.0-208.0 \\
208.0-218.0 \\
218.0-228.0 \\
228.0-238.0 \\
238.0-248.0 \\
248.0-258.0 \\
258.0-268.0 \\
268.0-278.0 \\
278.0-288.0 \\
288.0-298.0 \\
298.0-308.0 \\
308.0-318.0\end{array}$ & $\begin{array}{l}\text { Using } \\
\text { Using } \\
\text { Using } \\
\text { Usirg } \\
\text { Using } \\
\text { Using } \\
\text { Using } \\
\text { Using } \\
250 \\
200 \\
200 \\
200 \\
200 \\
200 \\
200 \\
200 \\
200 \\
200 \\
200 \\
200 \\
200 \\
200 \\
200 \\
200 \\
200 \\
200 \\
200 \\
200 \\
200 \\
200 \\
200 \\
200 \\
200 \\
200 \\
200 \\
200 \\
200 \\
200 \\
200 \\
200 \\
200 \\
200 \\
\text { De }\end{array}$ & 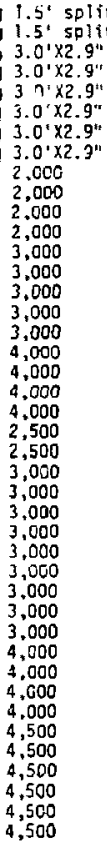 & $\begin{array}{l}\text { scoon } \\
\text { spoon } \\
\text { She iby tubes } \\
\text { She } 1 \text { by tubes } \\
\text { She } 1 \text { by tubes } \\
\text { Shelby tubes } \\
\text { Shelby tubes } \\
\text { Shelby tubes } \\
100 \\
150 \\
150 \\
150 \\
200 \\
200 \\
200 \\
200 \\
200 \\
200 \\
200 \\
200 \\
200 \\
200 \\
350 \\
300 \\
300 \\
300 \\
200 \\
200 \\
200 \\
200 \\
200 \\
200 \\
200 \\
175-200 \\
200 \\
300 \\
300 \\
300 \\
300 \\
300 \\
300 \\
300\end{array}$ & $\begin{array}{r}1.5 \\
1.5 \\
3.0 \\
3.0 \\
3.0 \\
3.0 \\
3.0 \\
3.0 \\
5.0 \\
6.0 \\
4.8 \\
4.2 \\
9.0 \\
10.0 \\
10.0 \\
8.0 \\
10.0 \\
2.0 \\
8.0 \\
10.0 \\
10.0 \\
10.0 \\
2.5 \\
9.5 \\
10.0 \\
10.0 \\
8.0 \\
10.0 \\
10.0 \\
10.0 \\
10.0 \\
10.0 \\
10.0 \\
10.0 \\
10.0 \\
10.0 \\
10.0 \\
10.0 \\
10.0 \\
10.0 \\
10.0 \\
10.0\end{array}$ & $\begin{array}{r}1.5 \\
1.5 \\
3.0 \\
3.0 \\
3.0 \\
3.0 \\
3.0 \\
1.7 \\
5.0 \\
4.7 \\
4.5 \\
4.5 \\
9.0 \\
9.7 \\
10.0 \\
8.0 \\
9.9 \\
1.7 \\
7.6 \\
10.0 \\
10.0 \\
9.8 \\
2.9 \\
9\end{array}$ & $\begin{array}{r}100 \\
100 \\
100 \\
100 \\
100 \\
100 \\
100 \\
57 \\
100 \\
78 \\
94 \\
107 \\
100 \\
97 \\
100 \\
100 \\
99 \\
85 \\
95 \\
100 \\
100 \\
98 \\
116 \\
96 \\
100 \\
100 \\
103 \\
98 \\
101 \\
97 \\
102 \\
99 \\
101 \\
100 \\
87 \\
100 \\
100 \\
100 \\
100 \\
100 \\
100 \\
100\end{array}$ \\
\hline
\end{tabular}




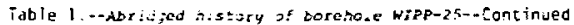

\begin{tabular}{|c|c|c|c|c|c|c|c|}
\hline $\begin{array}{l}\text { Core } \\
\text { No. }\end{array}$ & $\begin{array}{l}\text { Depth } \\
\text { intervai } \\
\text { in feet }\end{array}$ & RPM & $\begin{array}{l}\text { Weigit } \\
\text { on Git } \\
\text { (lbs) }\end{array}$ & $\begin{array}{l}\text { Circulating } \\
\text { pressure } \\
\left(1 b / \text { in }^{2}\right)\end{array}$ & $\begin{array}{l}\text { In } \\
\text { Feet } \\
\text { cored }\end{array}$ & $\frac{\text { erval }}{\text { Fee: }}$ & $\begin{array}{l}\text { Percent } \\
\text { recovered }\end{array}$ \\
\hline $\begin{array}{l}43 \\
44 \\
45 \\
46 \\
47 \\
48 \\
49 \\
50 \\
51 \\
52 \\
53 \\
54 \\
55 \\
56 \\
57 \\
58 \\
59 \\
60 \\
61 \\
52 \\
63 \\
64 \\
65 \\
66 \\
67 \\
68 \\
69 \\
70 \\
71 \\
72 \\
73 \\
74 \\
75 \\
76 \\
77 \\
78 \\
79 \\
80 \\
11 \\
82 \\
63 \\
84\end{array}$ & $\begin{array}{l}318.0-328.0 \\
328.0-33.0 \\
338.0-348.0 \\
348.0-35.0 \\
358.0-368.0 \\
368.0-374.0 \\
37400394.0 \\
394.0-393.0 \\
393.0-403.0 \\
403.0-413.0 \\
413.0-423.0 \\
423.0-433.0 \\
33.0-413.0 \\
443.0-451.0 \\
451.0-457.5 \\
457.5-458.0 \\
458.0-464.0 \\
464.0-466.5 \\
466.5-458.5 \\
458.5-47 . .0 \\
470.0-472.5 \\
472.5-492.0 \\
482.0-492.0 \\
492.0-502.0 \\
502.0-512.0 \\
512.0-522.0 \\
522.0-526.0 \\
526.0-533.0 \\
533.0-537.5 \\
537.5-564.0 \\
544.0-553.5 \\
553.5-563.5 \\
563.5-573.0 \\
573.0-593.0 \\
583.0-593.0 \\
593.0-603.0 \\
603.0-613.0 \\
613.0-615.0 \\
615.0-625.0 \\
625.0-635.0 \\
635.0-655.0 \\
645.0-650.0\end{array}$ & $\begin{array}{l}200 \\
200 \\
200 \\
200 \\
200 \\
200 \\
200 \\
200 \\
200 \\
200 \\
200 \\
200 \\
200 \\
200 \\
200 \\
200 \\
200 \\
200 \\
200 \\
200 \\
200 \\
200 \\
200 \\
200 \\
200 \\
200 \\
200 \\
220 \\
200 \\
200 \\
230 \\
200 \\
200 \\
200 \\
200 \\
200 \\
200 \\
200 \\
200 \\
200 \\
200 \\
200\end{array}$ & $\begin{array}{l}4,500 \\
4,500 \\
4,500 \\
4,500 \\
4,500 \\
4,500 \\
4,500 \\
4,500 \\
4,500 \\
4,500 \\
\because, 500 \\
4,500 \\
4,500 \\
2,000 \\
2,000 \\
2,000 \\
2,000 \\
2,000 \\
2,000 \\
2,000 \\
2,000 \\
2,000 \\
2,000 \\
2,000 \\
2,000 \\
2,000 \\
2,000 \\
2,000 \\
2,000 \\
2,000 \\
2,000 \\
2,000 \\
2,000 \\
2,000 \\
2,000 \\
2,000 \\
3,000 \\
3,000 \\
3,000 \\
3,000 \\
3,000 \\
3,000\end{array}$ & $\begin{array}{l}300 \\
300 \\
300 \\
300 \\
300 \\
300 \\
300 \\
300 \\
300 \\
300 \\
300 \\
300 \\
300 \\
300 \\
300 \\
300 \\
300 \\
300 \\
300 \\
300 \\
300 \\
300 \\
300 \\
300 \\
300 \\
300 \\
200 \\
200 \\
200 \\
200 \\
200 \\
200 \\
200 \\
200 \\
300 \\
300 \\
300 \\
300 \\
300 \\
300 \\
300 \\
300\end{array}$ & $\begin{array}{r}10.0 \\
10.0 \\
10.0 \\
10.0 \\
10.0 \\
6.0 \\
10.0 \\
9.0 \\
10.0 \\
10.0 \\
10.0 \\
10.0 \\
10.0 \\
8.0 \\
6.5 \\
.5 \\
6.0 \\
2.5 \\
2.0 \\
1.5 \\
2.5 \\
10.0 \\
10.0 \\
10.0 \\
10.0 \\
10.0 \\
4.0 \\
7.0 \\
4.5 \\
6.5 \\
10.0 \\
10.0 \\
9.5 \\
10.0 \\
10.2 \\
9.8 \\
10.0 \\
2.0 \\
10.0 \\
10.0 \\
10.0 \\
5.0\end{array}$ & $\begin{array}{r}10.2 \\
9.8 \\
10.2 \\
10.1 \\
9.9 \\
5.0 \\
10.0 \\
9.0 \\
10.0 \\
10.0 \\
10.0 \\
10.0 \\
10.1 \\
7.5 \\
6.2 \\
.4 \\
6.0 \\
.8 \\
1.4 \\
1.0 \\
2.5 \\
10.0 \\
10.0 \\
10.0 \\
10.0 \\
8.5 \\
3.8 \\
6.3 \\
3.8 \\
5.5 \\
10.0 \\
10.0 \\
9.5 \\
10.0 \\
10.2 \\
9.3 \\
10.2 \\
1.8 \\
10.1 \\
9.9 \\
10.0 \\
5.3\end{array}$ & $\begin{array}{r}102 \\
98 \\
102 \\
101 \\
79 \\
100 \\
100 \\
100 \\
100 \\
100 \\
100 \\
100 \\
101 \\
94 \\
95 \\
80 \\
100 \\
32 \\
70 \\
67 \\
100 \\
100 \\
100 \\
100 \\
100 \\
86 \\
95 \\
87 \\
84 \\
85 \\
100 \\
100 \\
100 \\
100 \\
100 \\
95 \\
102 \\
90 \\
101 \\
99 \\
100 \\
106\end{array}$ \\
\hline
\end{tabular}


Iable 2,--5tratigraphic 5umbery for borehole wrpp-25

\begin{tabular}{|c|c|}
\hline Rock unit & Jepth interval (feet) \\
\hline Fielstocene deposits" & $0-17$ \\
\hline Paritian rooks & \\
\hline Lewey Lake Red Beos & $27-232$ \\
\hline Rustler Formation & $232-565$ \\
\hline Gissolution residue & $270-287$ \\
\hline Magenta Dolomite Member & $302-328$ \\
\hline Oissolution residue & $4 ! 5-424$ \\
\hline Culebra Dolomite Member & $447-472$ \\
\hline Dissolution residue & $475-512$ \\
\hline Salado formation & $565-655$ (total dopth) \\
\hline Upper me,ner & $565-600$ \\
\hline Dissolution residue & $565-600$ \\
\hline 'MB 101 & .589 \\
\hline$\because \% 102$ & 1.599 \\
\hline salt incerval & $600-655$ \\
\hline${ }^{2} M B \quad 103$ & $\cdot 6: 5$ \\
\hline$=M B \quad 104$ & $n 628$ \\
\hline $3 ., 10105$ & -560 \\
\hline
\end{tabular}

- Depth interval recorded fram geophysical logs.

- Includes artificial fill for drill pad, gypsite, and alluvium.

Marker bed.

-Base of unit. 
As pact of the exploratory work, geophysical lo: ; were taken the full length of each borehole. The logging was done to facilitate the Ldentification and correlation of rock units and the identification of gross lithologies (i.e., dolomite, anhydrite, polvhalite, and rock salt), and to provide = depth determination independent of that indicated by dri11-rod measurement. For this borehole, the geophysical logs included (i) a gamma-ray curve that recorded vat iations in the distribution of porassium and other radioactive elements, (2) a gama-gama curve that recncited variations in rock density, and (3) a neutron cur ve that recorded variations in the distribution of hydrogen.

Borehole WIPP 25 penetrated sedimentary rocks totaling 655 feet in thlekness. The rocks include a younger group of continentai sediments of pleistorene age, and an older group of marine red beds and evaporites of permian age.

The Pleistocene sediments consist, in descending order, of an uniamed gypsito and unconsolidated alluvium. They have an aggregate thickness of 17 Eeet.

The Permian rocks include, in descending order, the Dewey Lake Red Buds, the Rustler Formation, and the upper part of the salado Formation. The Dewey lake is 2.15 feet of reddish-brown siltstone and mudstone ilberaliy bleached greenish-gray in spotty and splotchy masses, and abundantly intruded by crisscrossing and horizontal veins of fibrous selenite. The Rustler is an evapcrite Eormation, 333 feet thick, consisting chiefly of gypsum (derived from anhydrite), and poorly consolidated clay and silt (derived from clayey and silty halite), but contains interbeds of dolomite and k 11 - consolidated siltstone, as weil as residual pods and lenticlas of anhydrite. The salado is represented by 90 feet of evaporites and other rocks in WIPP 25, and includes two lithologically distinct parts--an upper, halite-free interval, and a lower halite-rich interval. T'in upper part is 35 feet of virtually unconsolidated clay and silt interlayered at irregular intervals with broken and shattered bands of gypsum and anhydrite; it is considered to be a sissolution residue derived from 260 feet of halite and associated rocks that were formerly present. Tne lower halite-rich part of the 
90 feet of the Salado in WIDP 25 is chiefly halite with tnin interbers of argillaceous halite, anhydrite and polyhalite. Additional stratigraphic details of the rocks are presented in Table 3 and Figure 2.

All measurements related to the drill site are in English units. These measurements include: The survey to locate the arill hole (both horizontally and veitically), the drilling depths as furnished by the Ariller, and the wireline logs as furnished by the logging company. Borehole location and depths ir the borehole are given in this report in English units. If metric units are cesized, the following conversion factors should be used.

\begin{tabular}{lcc} 
Multiply English unit & By & To obtain Metric unjt \\
\hline foot (ft) & 0.3048 & meter (m) \\
inch (in) & 25.4 & millimeter (mm) \\
pounds per square inch & 0.06895 & megapascal (MPa) \\
$\left(1 \mathrm{~b} / \mathrm{in}^{2}\right)$ & &
\end{tabular}

\subsection{HYDROLOGICAL DATA}

No nydrological data have been obeained to date from WIPP 25 .

\subsection{PEMARKS}

The first objective of the Nash Draw drilling program, to determine the stratigraphy of near-surface formations, is partially Eulfilled; this report now provides the stratigraphic data to the top of the Salado Formation, and a few marker beds within the upper Salado, at WIPP 25.

The second objective, to examine the relationship between subsurface structure and surficial features and deposits, will be fulfilled through the integrated interpretation of the Nash Draw surface and subsurface data in this and other reports.

The third objective, to investigate the dissolution of soluble rocks by groundwater, will be met through field nydrology tests and laboratory examination of dissolution products to determine, as possible, ages and genesis. The hydrological programs have not yet been undertaken; laboratory programs are just being initiated. 
Table 3.--ilthologic iog for borchole HIPP-25

(Color designations are from the Rock-Color Chart (Goddard and others. 1948); no core designates intervals where core was lost during drilling operations\}

Litholagic description

Depth interva? Feet

iypsite, yellowish-gray $(5 Y 8 / 1\}$, loose, unconsolioated, calcareous at

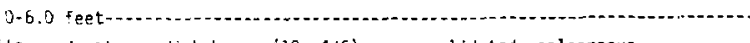

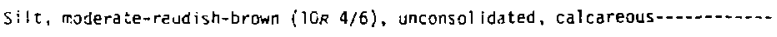

$5 i$ tstone, moderate-reddish-brown $\left(10_{R} 4 / 6\right)$ with reduction spots, hard,

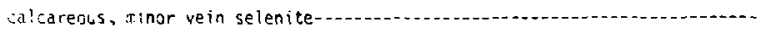

No core-1.2.

Mudstcre, moderate-reddish-brown $(10 R 4 / 6)$ to dark-reddish-brown $\left(10_{R} 3 / 4\right)$,

failiti: sanded lacally, unconsolidated in part, silty; contains moderate-

ye: in sm.ersen (1Jy $5 / 4$ ) reduction spots; some cross bedding at 28.0 -

27.: :ce:; oedcing̣ dios aporoximately $30^{\circ}$

$10.0-17.4$

$17.4-19.2$

$19.2-21.0$

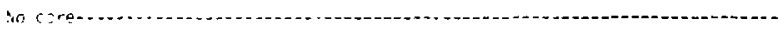

"udstone, -ojerzte-reddich-briwn (T0R 4/6), bery faintly banded locally;

cortairs a few scotiered reduction spots; greenish-gray (5GY $6 / 1)$ to

วतle-ye! iswish-brown (17\%R 6:2) reduction zone with $\mathrm{MnO}_{2}$ at 32.1-32.2

feet, rearl: bartisal, a-5-mm-thick vein of selenite joining a neally

hor:zontal, l-cmothick band of selenite at 34.7 feet; many 1-mm-thick seleni:e veins aipoing $20^{\circ}$ at interval 35.1-35.8 feet; horizontal,

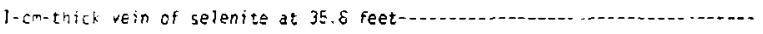

s11tstone, greenish-gray $(50$ r $5 / 1)$; contains linc $_{2}$ specks less than

i rs in clameter-a

5iltstone, cark-redtish-brown (10R 3/4): many 1-3-mm-thick selenite veins dipping at random angles, nearly vertical open fracture

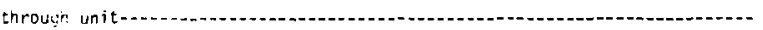

Silestone, dark-reddish-brown $(10 R 3 / 4)$; many $1-2-m m$-thick veins of elenite $0.5-1$ cm apart, mostiy horizontal but some dip at low to steeo angles; some $T-c_{1}$-thick bands of mudstane; many greenish-gray $: 5 . \cdots 6,1)$ reduction spots

Wdstone, Jark-reddish-brown $\left(10_{R} 3 / 4\right)$; contains wany low-dipping.

-z-m-inick veins of selenite, most $0.5-1 \mathrm{~cm}$ aparti a few $1-\mathrm{cm}-$ :rick bands of dark-raddish-brown $\left(10_{R} 3 / 4\right)$ siltstone and many $1.4 \mathrm{~mm}$ in 1 ireter, greenish-gray $(5 \mathrm{Gr} 6 / 1)$ reduction spots-.....

517 stone, dark-reddish-brown $(10 R 3 / 4)$, faintly banded, some crossbedding; contains greenish-gray $(5 c \gamma 6 / 1)$ reduction spots $1-3 \mathrm{~mm}$ a-jeter, irregular, discontinuous, greenish-gray $(5 c y 6 / 1)$ reduction zone at 44.8-45.2 feet; many 1-3-mm-thick selenite veins = 30ing randomly from $0^{\circ}$ to $60^{\prime}$; a $3-\mathrm{cm}$-thick harizontal band af ziorjess to white $\left(\mathrm{w}^{9}\right)$ selenito at $4 \uparrow .8$ feet; a $5-\mathrm{cm}-\mathrm{thick}$ Green ish-gray (5GY $6 / 1)$ reduction zone at 41.3 feet; a 2 -cm-thick band of dark-reddish-brown $\left(10_{R} 3 / 4\right)$ mudstone sandwiched between $\therefore$ j-m-tnick horizontal band of selenite above a $2-\mathrm{cm}-\mathrm{thick}$ morizontal band of selenite at $\mathbf{4 3 . 6}$ feet- 
Table 3.--Lithologie log for borehole wipp-25--Continued

Lithologic description $\quad \begin{gathered}\text { Depth interval } \\ \text { Feet }\end{gathered}$

Mudstone, dark-reddish-brown ( $1083 / 4)$; crossbedded, contains $0.2-0.8-$ foot-thick bands of siltstone as in unit at 41.0-45.2 feet, many greenish-gray $(5 G y 6 / 1)$ reduction spots, random selenite veins dissirg $0^{\circ}$ to $60^{\circ}$; cut-and-fill structures from 47.8 to 50.2 feet (into init below) -

$45.2-50.0$

Siltstone, joderate-reddish-brown $(10 R 4 / 6)$ to dark-reddish-brown $(10 \Omega 3 / 4)$, finely laminated, crossbedded cuntaine 1-?-mm-diameter grayish-green $(\log y 4 / 2$ ) reduction spots throughoul, 1-2-mm-thick veins of selenite at random angles and nearly norizontal, 1-4-cmthick bands of selenite every $1-2$ feet, some small mud clasts, and a few cut-and-fill structures throughout; a le - con thick of very fine grained to medium-grained, moderate- $r$; di $i$-brown (10R 4/6) sandstone at 58.8 feet; a $2-\mathrm{cm}-$ thick similar sandstone lens at 59.4 feet; a 2-cm-tnick band of dark-greenish-gray ( $5 \mathrm{CY} 4 / 1)$, silty clay at 59.5 feet cut by a $20^{\circ}$-dipping, $i-c m-t h i c k$ vein of sele.ite---n--...-

$50.0-59.7$

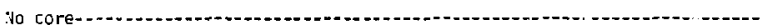

Eiltstone, maderate-redd, sh-brown $(10 \pi 4 / 6)$ to dark-reddish-brown $(10 R 3 / 4)$, thinly laminated with dark-reddish-brown (10R 3/4) mudstane; crossbedding common, bedding somewhat wavy in places; conteins meny 1-3-rm-diameter, grayish-green (10cy 5/2) reduction sjots (few up to $2 \mathrm{~cm}$ in diameter); numerous ( $T-2$ per $\mathrm{ft}$ ) $4-30-\mathrm{mm}$ thick veins of selente, most dipping from $5^{\circ}$ to $10^{\circ}$, but a few have $65^{\circ}$ dips and a faintly banded, dark-reddish-brown $(10 R 3 / 4)$ mudstane sean with reduction spots at 62.0-62.7 feet, open fracture dipping $70^{\circ}$ at 72.0 feet; thro irregular mud-filled fractures dipping 50" at 76.8 feet; continuous, slightly irregular, grayishgreen $(10 \mathrm{Gr} 5 / 2)$ siltstone band at 79.2 feet-_...

Siltstone, noderate-reddish-brown $(10 R 4 / 5)$, crumbly, with soft, roderate-reddish-brown $(10 R 4 / 6)$ to grayish-red $\left(10_{R} 4 / 2\right)$ mudstone; few greenish-gray $(10 G y 5 / 2)$ reduction spot3-....... 88.0-89.7

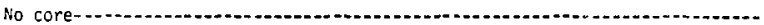
Siltstone, same as unit at 60.0-87.9 feet; irregular, 3-cm-thick, light-alive-gray $(5 \mathrm{r} 6 / 1)$ siltstone band at 92.0 feet; thin selenite veinlets at 93.7 seet; 1 -cm-thick selenite vein dipping $65^{\circ}$ at 97.0 ceet; open fractures dipping $55^{\circ}$ at 91.7 feet and $10^{\circ}$ at 94.8 feet;

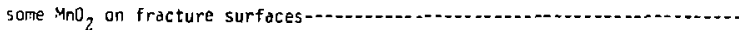


TuDle 3.--Lithologit log for borehole HIPP-25--Continued

Lithologic description

Depth interva) Feet

Siltstone, sare as unit at $60.0-87.9$ feet; crossbedded; contains numerous i- I-nmmich veinlets and tands of seienite throughout; several highangle fractures realec with selenite; vertical selenite-healed frastures connect bedding olanes at $115.8,116.5,120.6$, and 121.8 feet; continuous, faintiy banded silcstone grading to very fine grained sandstone bards at 102.1-102.4 and 102.8-103.0 feet large irregular, grayishgreen (106:5.2) alteration spots at 101.6-101.7, 104.2-104 4, and 105.4\$05.6 Feet: $1-10 \mathrm{~mm}$ aands and lenses of very fine grained to mediumgrained sandstone, interbedded with siltstone from 106.0 to 114.0 feet; dip varies from $3^{\prime}$ to $10^{\circ}$; faintly bedded, very fine grained to medium-grained, grayisn-green $(10 \mathrm{CY} 5 / 2)$ to light-olivemgray $(5 \mathrm{r} 6 / 1)$ sands tone bands at $11.7-112.8$ and $113.3-113.6$ feet, also cut by selenite veins; irregular, grayish-green $(10 G 55 / 2)$ reduction zones at $122.7-$ 122.3 ind $124.6-125.0$ fpet--0.0 $99.0-125.0$

Mustone, dark-reddish-brown ( $\left.O_{R} 3 / 4\right)$, interlaminated with siltstone ut $126.5-126.9$ feet; contains raduction spots and few hairline

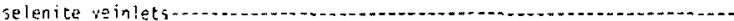

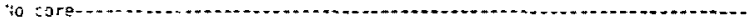

udstone, rederate-recdish-brows $(10 R 4 / 6)$ to dark-reddish-brown

(:GR 3.4): very fow scattered i-3-rT-diameter, grayish-green

$\{1,5,5,7)$ reduccion $5=0 t s$ generally concentrated between 133.3 and

135.8 feet: anj thin, less than 1-mm-thick gypsun veinlets; $\mathrm{MnO}_{2}$ * Dating, diag:ng $10^{\circ}$, at 30.2 feet; unit becones slightily silty

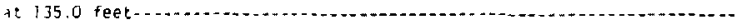

$128.0 \cdot 140.9$

Silitszone, mcderate-reddish-brawn $\left(10_{R} 4 / 6\right)$ to dark-redjish-brown (!OR 3/4): contains few faint, wayy, dark-reddish-brown $(10 R 3 / 4)$ fudstone laminae; 1-3-mi-dianeter, grayish-green (lacr 5/2) reduction 5fois, many 1-2-mm-thick veins of seienite dipping $5^{\circ}$ throughbut unit at incervals of $5 \mathrm{mi}$ to $3 \mathrm{~cm}$, few scattered, nearly vertica? selenite veinlets connect bedding pianes; bottom 2 feet of unit tyrades to audstone.

$140.9-150.0$ 
Table 3.--Lithologic log for borahole wIPP-25--Continued

Lithalagic description

Cepth interval

Faet

Siltstone, moderate-reddish-brown $(10 R 4 / 6)$ to dark-reddish-brown (10R $3 / 4)$, some bands trending toward dark-reddish-brown (10R 3/4) mudstone;

1-3-הr-dianeter, grayish-green (10GY 5/2), randomly dispersed reduction

5FOis; numerous wavy, continuous, nonparallel, 1-2-mm-thick translucent selenite veinlets, dipping $0^{\circ}-5^{\circ} ; 2$-cm-thick light-red $(5 R 6 / 6)$ mudstone banu dipping $15^{\circ}$ with a few soft, chalky, 2-3-mm-diameter, white (N9) to vecy light gray ( $N 8$ ) nodules at 152.0 feet; mud-filled fracture dipoing 35 - at 153.3 feet; irregular network of gypsum-filled fractures at 154.0 -

154.3 feet along with interconnecting apen conjugate fractures dipping $20^{\circ}$ and $65^{\circ}$; mudstone band at 155.2-155.5 feet, top contact romizontal, bottom dipoing $35^{\circ}$; horizontal mudstone band at 156.3-156.5 feet; increasing number of nearly horizontal gypsum veinlets at 156.6 feet----..--

silestone, same as unit at 150.0-160.0 feet; contains 1-3-mm-thick,

$150.0-160.0$ greenish-gray :5GY b/1) siltstune laminae at $161.5,161.8$, and 162.2 feet, 2-cm-diameter reduction spot at 163.8 feet, 5-m-thick selenite vein dipping $55^{\circ}$ at 166.0 feet; horizontal, 2-cri-thick mudstone band dt 167.8 feet-

silestone, roderate-reddish-browh $(10 R 4 / 6)$ to dark-reddish-brown $\left(1 C_{R} 3 / 4\right)$, grading to mudstone; contains trace of $\mathrm{MnO}_{2}$, grayish-green (10.3i 5;2) reduction spots $1-3 \mathrm{~mm}$ in diameter; few nonparallel, continuous, 1-2-rm-thick selenite veinlets dipping $0^{n} \cdot 5^{n} ; 1.5-c m-$ thick, roderate-reddish-orange $(10 R 6 / 6)$, nodular-appearing band of sucstone at 169.2 feet; two selenite veins $3 \mathrm{~cm}$ apart, dipping $50^{\circ}$, connected by conjugate veinlets at 169.8 feet; selenite veins dioping $55^{\circ}, 60^{\circ}$, and $65^{\circ}$ at $171.8,172.0$, and 172.1 feet; two intersecting selenite veins dipping $55^{\circ}$ and $45^{\circ}$ with 1 -cm-long branching selenite vein at 173.0 feet; selenite veins dipping $70^{\circ}$ and $\mathrm{BO}^{\prime \prime}$ at 174.0 and 177.0 feet-1 1 tone. same as unit at $168.2-178.0$ feet; sparse 1-4-mm-diameter (sone $1 \mathrm{~cm}$ in diameter). grayish-green (10Gy 5/2) reduction spots concentrated at 181.3-181.6 feet; intersecting, continuous, 1-3-fmthick selenite veinlets telow 184.0 feet; dark-reddish-brown \{110R 3ia) mudstone at 178.0-i79.5 feet; selenite veins dipping 65" and $55^{-}$at 178.2 and 184.8 feet; open high-angle fracture at

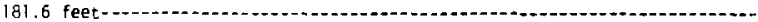


Table 3.--tichologic lag far borehole HIPP-25--Continued

Silts tome, moderate-reddish-brown $\left(10_{R} 4 / 6\right)$ to dark-reddish-brown $\left(10_{\lambda} Z^{\prime} 4\right)$; grades to mudstone in lower foot; mane scattered 1-3-mm-diameter. grajishgreen $(10 \mathrm{c} \% 5 / 2)$ reduction spots (concentrated at 196.8-197.0 and 197.3197.4 ft); 1-2-mm-thick, continuous, intersecting selenite veinlets (concentrated in 192.6-193.5-ft interval); grayish-red (10R 4/2) mudstone with few 1-2 mm dark-raddish-brown (10R 3/4) mud clasts at 190.0-190.] feet: highly fractured and byiken zone at 191.8-192.1 feet; two fractures

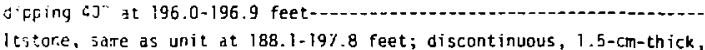
grayish-green (10Gy 5/2) siltstone band at 198.0 feet; highiy broken, dark-reddish-brown (10R 3/4) mudstone at 205,4-206.0 feet; scattered seienite veins dipping $15^{2}-20^{\circ}$.

i itstone, ioderate-reddish-brown $(10 R 4 / 6)$ to dark-reddish-brown $(1,303 / 6)$ nattled appearance; numerous thin cross-cutting selenite vinle:s: icres of 1-3-mm-diameter mudstone fragments in a mud atrix at 2:0.5-211.4 and 213.9 feet; partings alung selenite veins jip 32 ; numerous $i-4-m m-d i a m e t e r$, greenish-gray (10, $5 / 2)$ reduction spots; locally few faint, irregular, discontinuous laminae of Jark-reddish-brown $(10 \mathrm{R} / 4)$ mudstone-10.-. $209.7-217.9$

Silistone, moderate-reddish-brown $\left(10_{R} 4 / 6\right)$ to dark-reddish-brown $\left(10_{R} 3 / 4\right)$, grading to mudstone at 226.0-228.0 feet, many 1-2-mm-thick continuous, nonparallel veins of selenite and 1-4 mn grayish-green (10\%5/2) reduction spots (some as large as $1 \mathrm{~cm}$ in diameter): 3-cm-tnick, mottled grayish-green (10Gr 5/2) and dark-reddish-brown (10R 3/4) siltstone bordered by 1 -mm-thick selenite veins dipping $30^{-}$at 221.2 feet; most selenite veins dip from $0^{\circ}$ to $15^{\circ}$, snme dip 30

Mids:one, moderate-reddish-brown (10R 4/6) to dark-reddish-brown

( liak 3,4$)$. soft to moderately hard, some fissile; $1-4 \mathrm{~mm}$ ir

14i.weter, Jrayish-green $(10 \% \% 5 / 2)$ reduction spots; slightly wavy and norizortal contact with siltstone unit above;0.5-cm-thick greenish-gray \{Si $5: 11$, soft siltstone at base of unit, slightly wavy horizontal

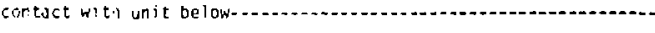

Lypsum, i ght-olive-yray $(5: 6 / 1)$. massive, very finely crystalline. slightly anhydritic; numerous specks 2-3 $\mathrm{im}$ in dianeter, very numerous and dispersed bands giving mottled appearance; irregular, interconnecting, 1-2-rm-thick, Toderate-reddish-brown (10R 4/6) 
Table 3.-.Lithologic Iog for borehole wrPP-25--Continued

Lithologic description

Lypsum, light-olive-gray $(5 \% 6 / 1)$ and olive-gray $(5 \gamma 4 / 1)$, massive to very

finely crystalline; upper contact dips $10^{\circ}$; wavy, continuuss, nonparallel; indistinct bands give core a "chicken-wire" or nodular appearance; few veinlike structures dipping $5^{\circ}-12^{\circ}$; wo $4.5-\mathrm{cm}-d i a m e t e r$, massive, medium. lignt-cray (v6) to light-olive-gray (5r 6/1) anhydrite masses al

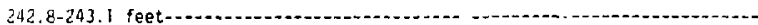

Gjpsum, medium-light-gray (N6) to light-o.ive-gray $(5 Y 6 / 1)$, very finely

crystalline, with many 1-2-mm-thick, wavy, branching veins of gypsum,

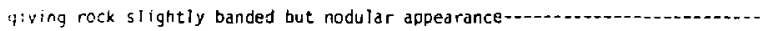

$233.7-243.7$

Gyssum, sarke as unit at $233.7 \cdot 243.7$ feet-........

$243,7-244.6$

$244.6-245.7$

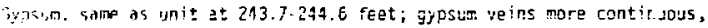

less branching, giving rock more of a banded appearance-_...

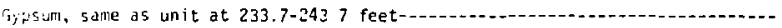

jo core-1.-2

$245.7-246.6$

$246.6-246.7$

$246.7-248.0$

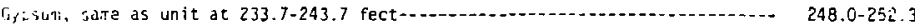

hinh drite light-olive-gray $(5 r 6 / 1)$ to mediun-light-gray (.06), very

finely crystalline, wavy gypsum veins as thick as $1 \mathrm{~mm}$, most veins

are horjzontal but a few low angle; veins become tnicker and more

numerous and branching, giving anhydrite a slightiy elongate nodular

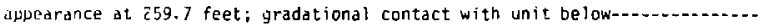

jysur, I ignt-olive-gray $(5 \% 6 / 1)$ and ol ive-gray $(5: 4 / 1)$, very finely

crystalline, slightly anhydritic, speckled appearance caused by

1-2-rnl-diameter dark specks; dispersed banding gives mottled

appearance; irregular, light-olive-gray (5Y 6/1) anhydrite band at

262.6-262.7 feet; wavy, 2-3-mm-thick, light-oliven:;ay (5Y 6/1)

siitstore band at 261.9 feet; iminated, slightly dolomitic, light-

olive-gray $(5 \% 6 / 1)$ siltstone band at 266.2-266.3 feet; 2-4-mm-thick,

slit, wavy, almost horizontal gypsum-filled fracture at 262.2 feet-........

$260.0-269.5$

Hudstone, light-brownish-gray (5iR 6/1) to brownish-gray (5YR 4/1);

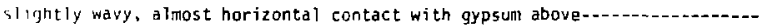

Mudstone. moderate-reddish-bruwn (10R 4/6) to dark-reddish-brown

$(13 \pi 3 / 4)$. silty, 1-5-mim-wide colorless to white (N9) fragments

of jypsum scattered randonly throughout; irregular, wavy, roderate-

reduish-brown $(10 R 4 / 6)$ to dark-reddish-brown (I/R 3/4) siltstone

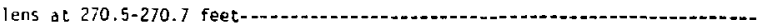

$269.6-271.9$

Silt, moderate-reddish-brown (10R 4/5) to dark-reddish-brown (10R $3 / 4)$.

yrading to clay, unconsolidated, faintly banded ocally, with thin, discontinuous, wavy, dark-reddish-brown (10R $3 / 4 i$ clay laminae; few scattered 1-2-rm-diameter fragments of white ( $\mathrm{N} 9)$ gypsum in upper part of unit; few $1 \mathrm{~mm}$ or less in diameter, grayish-green (10GY 5/2) reduction spots; thin parting dipping $40^{\circ}$ at 272.5 feet; $3-4-m$ thick, two conjugate veins of selenite, dipping $50^{\circ}$ and $70^{\circ}$ at intervai 239.2220.0 feet; unit becomes harder and better consolidated at 282.2 fee:--...- 
Jable 3.--Lithologic log for borehole wrpp-25--Continued

Siltstcne, paie-brown $(5 ; \% 5 / 2)$ and light-0live-gray $(5 y 5 ; ?)$, conglomeritic (1-is m:, rounded, ight-olive-gray $(5 Y 5 / 2)$ silestune and white (N9) gypsum fragrents in light-ol ive-gr?v $(5 y 5 / 2)$ silty matrix). cut jy numerous, 1-2-Tam-thick, ilreqular, wavy, branching gypsum veins

Siltstone, pale-brown $\left({ }^{4}{ }_{Y R} 5 / 2\right)$, yiduish-brown $\left(5_{Y R} 3 / 2\right)$ and light-olivegray $(5 \mathrm{~F} 5 / 2)$, irregular and wavy banaing, cut by numerous 1-2-mm-thick, irregular, wavy, branching gypsum veins; slightly dolomitic: bedding dips $30^{\circ}-$

Gypsum, light-olive-gray $(5 \times 6 / 1)$ to olive-gray $(5 \times 6 / 1)$, very finely crystali ine, slightly mottled and showing vein structures; contact between gypsum and unit above is irregular, with few 5 mall gypsum fragments suspended in siltstone; 1-2-mm-thick wavy bands of grayishbrown (5YR 3/2) silty gyosum dipping $40^{\circ}$

$286,5-239.0$

5yfsum, light-olive-gray $(5 \mathrm{r} 6 / 1)$, ol ive-gray $(5 y 4 / 1)$, ol ive-black

$: 5 r 2 / 1)$ and grayish-red $(10 F$ 4/2) with dark-yellow $(5 y, 6 / 4\}$ cint, roz:led, very finely crystalline, way and irregular banding; few i-lis-rri-trick, randanly scattered white $(49)$ gypsum veins throughout; 5i: : . Grjyish-brown $(5 \% 93 / 2)$ bands at 292.2-292.5, 296.2-297.2, and 3il.j-jC1.7 feet; nunerous !-2-mm-Jiameter selenite crystess in a cerate-brown (5Ya 3/4) and dark-yellowish-orange (10,R 6/6) to

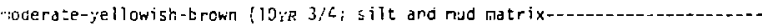
Eciarite end gjosum; dolcrite, ol ive-gray $(5 y \$ / 1)$ to light-olive-gray

i5: 5: i; 5oric pale-yellowish-brown $(10 \mathrm{rR} 6 / 2)$, silcy, numerous, 1-mmdiarster, randomily dispersed selenite crystals; roct has a speckled and banded appearance; gypsum, olive-black $(5 \times 2 / 1)$ to olive-gray $(5 y 4 / 1)$ and white ( $(9)$ to colorless horizontai bands-a $302.2-308.4$ Dotomite, light-olive-gray $(5 Y 6 / 1)$ to olive-gray $(5 \% 4 / 1)$ and minor pale-brown $(5 i R 5 / 2)$; banded white $(N 9)$ t colorless gypsum veins at 308.4-309.1 and 317.0-320.7 feet; gypsum veins in an olive-gray $(5: 4 / 1)$ and datk-reddish-brown (10R 3/4) argi3laceous dolomite at 310.2-3:0.8 feet; horizontal alive-gray (5r 4/1) gypsum band and 1-m selenite crystal speckled rock at $313.6-313.8$ feet; moderatereddish-bro:m (10R $4 / 6)$ to pate-brown $\left(5 r_{R} 5 / 2\right)$ argillaceous dolomite at 315.0-315.1 feet has gradational contacts with dolonite above and beiow; lower 3.5 feet has alternating bands $1-13 \mathrm{~cm}$ thick of grayishred (5P 4/2), lignt-o? ive-gray (5y 6/1), and olive-gray (5Y 4/1) 
Taole 3.--Eithologic log for borehale wrPp-25--Continued

Dotorit $\rho, 1$ ight-olive-gray $(5 \times 6 / 1)$ and olive-gray $(5 \times 4 / 1)$, and grayish-red (5R $4 / 2$ ), wavy 1 aminae; lower 0.6 foot lacks the grayish-red (1OR 4/2); aypsum-filled fracture dipping $50^{\circ}$ at 323.1 feet has $5-(\pi m$ vertical disFlacement; minor white $(\mathrm{w} 9)$ to colorless gypsum laminae at 324.2-326.4 feet, gyosum-filled fracture dipping $55^{\circ}$ at 328.5 feet has $4-\mathrm{mm}$ vertical displdcement

aypsum, gypserferous anhydrite and anhydrite remants; gypsum, olive-black $(5 Y 2 / 1)$, olive-gray $(5 Y 4 / 1)$, dark-yellowish-brown $(10 Y R 4 / 2)$, and dtsky-jellowish-brown $(10 Y R 2 / 2)$; mottled appearance, numerous crisscrossing veinlets of white ( $N 9$ ) gypsum. Gypersiferous aniydrite, olivebiack $(5: 2 / 1)$. olive-gray $(5 \% 4 / 1)$, medium-1ight-gray $(.25)$ and derkyellowish-brown (10YR 4/2) and dusky-yellawish-brown (10YR 2/2), f nely crystalline, mottled ("chicken-wire") appearance, numerous white ( $\mu 9$ ) crisscrossing veinlets, gypsiferous anhydrite in the following intervals: 5i3-324, 356-359, 370-372, and 384-386 feet. Anhydrite remant, olivegray $(5 y 4 / 1)$ and olive-biack $(5 \times 2 / 2)$, finely crystalline, anhydrite $36 ? .3-364.5$ feet; moderate-red $(5 R 4 / 6)$ to moderate-reddish-brown (10:R 4;6) clay blebs at $329.9-331.0$ feet: remnant dolomite 328.8-330.4 feet; dolomite nodules and lenses separated by gypsum veinlets at 331.9 .332 .1 feet. Gypsum-healed fractures dipping $75^{\circ}$ at $349.5,40^{\circ}$ at 369.2 , $45^{\circ}$ at 375.1 and $376.2,80^{\circ}$ at $376.7,70^{\circ}$ at $377.2,20^{\circ}$ at 383.8 and 387.7

fez., רurerous $60^{\circ}-80^{\circ}$ at $390.0-391.0$ and two $50^{\circ}$ at 413.7 feet; 3-cmthick medium-crystalline gypsum band at 373.1 feet and a $1.5 \mathrm{~cm}$ band at 373.6 feet. Laminae at $379.7-383.5$ dip $20^{\circ}, 45^{\prime}-50^{\circ}$ at $401.2-402.6$, $50^{+}$at $406.0-413.0$ feet $328.8-415.7$

Hudstone breccia, medium-gray $(N 5)$, wavy to folded oljue-gray $(5 \times 4 / 1)$ ianinae; some moderate-brown ( 5 rR 4/4) mud; unit contains about 5 percent gypsiri as thin to thick, irregular, discontinuous bands and elongate, anguiar fragments; 3-cm-wide gypsum band dipping $40^{\circ}$ at 418.0 feet-2-0.-1

$415.7-418.2$

Muss cone breccia, medium-gray (N5), soft, thin (less than I min), short, faint, discontinuous, moderate-brown ( $\left.5 \mathrm{H}_{R} 4 / 4\right)$ lamuae and many sral i ( $1 \mathrm{~mm}$ ), wnite ( $\mathrm{Ng}$ ) gypsum fregments; $i-2-m m-t h i c k$ gypsum band

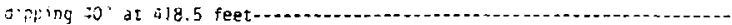


Table 3.r-2ithologic log for bazehole HIPP-25--Continued

Lithologic description

Depth interval Feet

luds tone breccia, moderate-brown $(5 Y R 4 / 4)$ to dark-reddish-brown $\left\{10_{R} 3 / 4\right\}$, with some nedium-gray ( $N 5$ ); 1-4-mm-diameter fragmants of white ( $N 9$ ) gypsum throughout, giving rock a brecciated appearance; l-cm-thick gypsum band $d^{2}$ poing $5^{\circ}$ at 419.4 feet; 3 -cm-thick gypsum band dipping $2^{\circ}$ at 420.0 feet: rany 2-5-cr-didreter fragrents of gypsum (made up of broken, discontinuous jands of gypsum\} at $122.0-423.0$ feet; $15-\mathrm{mm}$-thick band of grayish-pink (5:73/2) tc white ( $\because 9)$ magnesite(?) at 424.2 feet; mud and gyosum contact

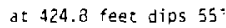
$419.2-424.8$

by fsum, olive-gray $(5 \mathrm{r} 4 / 1)$, very finely crystalline, remnant anhydrite;

many 1-2-mm-thick, irregular, branching, gypsum veins throughout, langest at $425.3-425.4$ feet-

$424.8-428.0$

Gyosum breccia, olive-gray $\left(5 r^{4} / 1\right)$ and dusky-yellowish-brown (10YR 2/2); rish jusky-brown (5/R 2/2) and some dark-redjish-brown (10R 3/4) mud as fracture fill and as matrix around gypsum fragments at 428.0-430.5 feet----Gyosum, olive-gray $(5 x 4 / 1)$, numerous $1-4-m m-t h i c k$, continuous and discontinuous, wavy laminae of selenite dipping $0^{\circ}-15^{\circ}$; many $1-3-\mathrm{mm}-$ wide, dusky-yellowish-brown (10re 2.2) gypsum blebs inhydrite, recium-iight-gray $(N 4)$, finely crystalline, numerous 1-2 тor, kavy, dusky-brown (5iR 2/2) gypsum veinletsGypsiferous anhydrite, olive-gray (5y 4/1), patches of white (N9) and dark-yellowish-brown (10YR 4/2) very finely crystalline; colorless to blue-t inted gypsum crystals $1-5 \mathrm{~cm}$ at $440.0-440.5$ and $440.8-441.2$ feet-----Gypsim, ulive-gray $(5 y 4 i)$ ), very finely crystalline; colorless to bluetinted gypsum crystals $1-5 \mathrm{~cm}$ at $444.8-445.7$ feet-

Yud-ciaystulie, dusky-brown $\left(5 y_{R} 2 / 2\right)$ and moderate-yellowish-brown (lo:R $5 / 4$ ) to dark-yeliowish-brown (love 4/2), soft, dolomitic, highly fractured; top contact horizontal, bottom contact dips $25^{\circ} \ldots . . . . . . . . . . . .$.

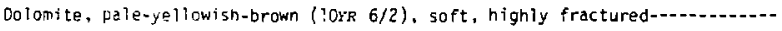
Dolomite. Iight-olive-gray (5y $6 / 1)$, very finely crystalline, highly

fractured- .........

id core-.

Dolonite, sate as init : $5490.0-450.5$ feet, but fractures are filled with pale-yellowish-brown $(10 \% 6 / 2)$ mud; core broken up into pebble-

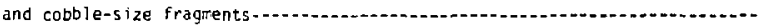
No core--1-0 Solorite, sare as unit ac 451.0-457.2 feet-

No core-1...

Colomite, same as unit at $45: .0-457.2$ feet; fragments range from $2 \mathrm{~mm}$ to 0.5 foet

$446.8-447.5$ $447.5-449.0$ 449.0-450.5 $4505-4 \dot{51} \mathrm{C}$

$457.0-457.2$ $457.2-457.5$ $457.5-457.9$ $457.9-458.0$ 
Table 3.-Aichologic log for borehole wrpp-25--Continued

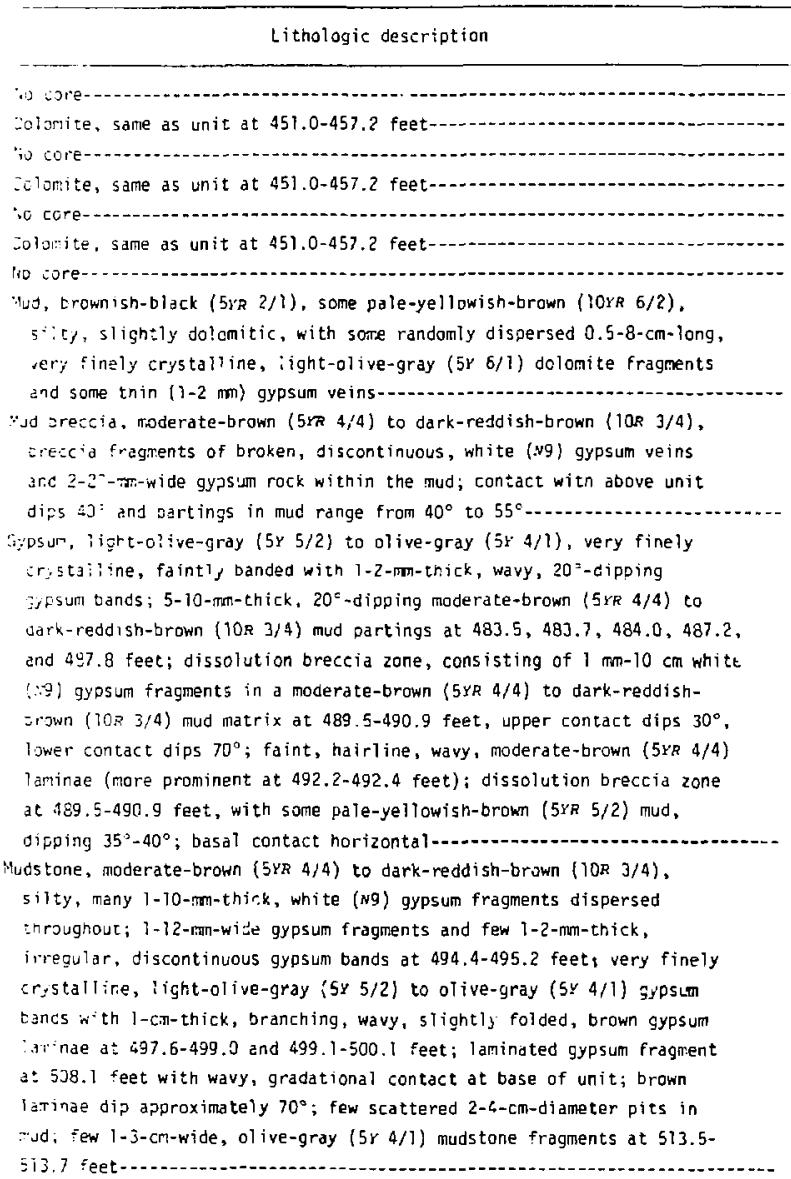

Depth intervai Feet

$464.8-466.5$

$466.5-467.9$

$467.9-468.5$

$468.5-469.5$

$469.5-470.0$

$470.0-471.2$

$471.2-472,0$

$472.0-475.3$

$475.3-432.0$

$482.0-493.7$

493.7-513.7 
Tatle j.--i:rho:ayic iog tor borehoie w1Pp-25--Continued

-itnologic description

Septh interval

Feet

cerastcre, olive-gray $(5: 4 i 1)$ to medium-dark-gray (a4), very cinely g"aned: silty; wavy, laxinated bedding; grades into a wavy laminated to folded, very finely grained, roderate-reddisi-brown (10R 4/6) to jark-redoish-brown ! 10 s 3/4) sandstone witn few scattered, short gyps:m veinlets and $1-4-1$ m-wide gypsumi fragments; pedding dips $70^{3}$

wd. 7oderate-brown ( $54 / 4$; to derk-reddisri-brown (10R 3/4) "Jumbo"

clay a: $419,2-52 \mathrm{C}$. A feet

in ior

Had. same as unit at 515.9-520.6 feet, 6-cm band of mud containing

$1-c " 1-1$ ong, anjular, pale-brown $(5 \% R 5 / 2)$ mudstone fragments at

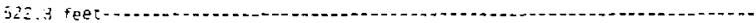

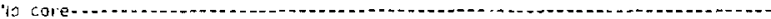

$\because .0$. sal:e as unit at $516.9-520.6$ feet

*a, rark-greanisr-gray (5G 4/1), grading into a dark-gray (N3), roderace-reddish-brown $(10 R 4 / 6)$ to dark-reddish-brown (10R 3/4)

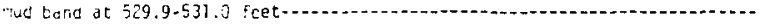

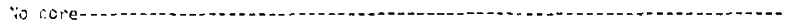

$\because$ A. calk-greerish-gray $(564 / 1)$, grading to greenish-black $(502 / 1)$, some roderate-reddish-brown $(10 R 4 / 6)$ to dark-reddish-brown (10R 3/4)

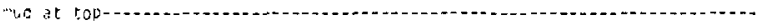

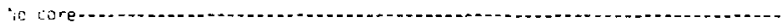

"ucs:one, Jark-greenish-gray $(50 y 4 / 1)$; hard, angular, sijty, mudstone

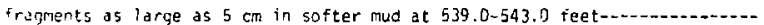

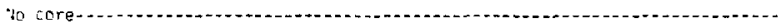

Mudstone. dark-greenish-gray $(564 / 9)$, soft to fim: trace of moderatetrown $\{5: P 3 / 4 ;$ silc, rarbled through aud at 544.0-567.0 feet: irregular roderate-trown (Eir $3 ; 4)$ mid band at 557.5-557.9 feet i irregular, siscontinuous, siiçntly calcitic, gypsum stringers dt 557.2-558.2 feet---.-ذј: feliowisr-brown $(i j: R 5 / 4)$ and sore lignt-olive-gray $\{5 \% 6 / 1)$, mottled, firely crystalline, high-angle, thin, wavy laminae; brecciated, fragments angular and less thar 1 गग to $7 \mathrm{~cm}$ by $12 \mathrm{~cm}$ size range in a moderate-

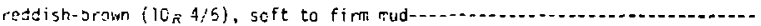

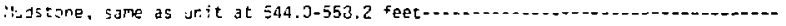

Hudstone breccia, Toderate-brown $(5 \mathrm{rg} 3 / 4)$ and moderate-reddish-brown (10:- \$;6;, soft to $r$ int; roderate-reddish-brown $(10 R 4 / 6)$, darkjellowisr-brown $(: 0 ; 04 / 2)$ and light-Dlive-gray $(5 \% 6 / 1)$, mottled, medium-crystali ine. some argiliaceaus, brecciated, gypsum fragmerts iess ctian 1 im to $5 \mathrm{~cm}$ thick: some mudstone is dark-greenish-gray (5: $4 / 1)$ as inclurions and some maroled through "brown matrix" mud; trace noderately hard, dark-greenish-gray (504/1) silt fragments; less gypa... and more dark-greenish-gray (5 $5 \% 4 / 1)$, subangular silt inclusions at bs.3-581.5 feet 
Table 3.--itibologic log for berehole hipp-25--Continued

Lithologic description

Depth interval Feet

Siltstone, moderate-reddish-brown (10R 4/6), soft, crumbly, with moderatereddisn-brown $\left(10_{R} 4 / 6\right)$ to dark-reddish-brown $(10 R 3 / 4)$ clay and numerous 1-3-mm-diameter, light-bTuish-gray $(587 / 1)$ silty blebs; 51 ightly wavy, light-hluish-gray $(5 a 7 / 1)$ silt band dipping $12^{\circ}$, with 1-3-mm-thick, mode rate-reddish-brown $(10 R 4 / 6)$ silt stringers at $584.8-585.0$ feet; blebs and stringers of soft,pliable, medium-bluish-gray $(5 B 5 / 1)$ silt in 2 Toderate-reddish-brown $(10 R 4 / 6)$ to dark-reddish-brawn $(10 R 3 / 4)$ mud in šsal $4 \mathrm{~cm}$

$58 ? .5-587.0$

jysurn and aitered polyhaite, moderate-reddish-orange $\left(10_{R} 6 / 6\right)$, rccerate-reddish-brown $\left(10_{R} 4 / 6\right)$, moderate-reddish-orange $(10 R 6 / 6)$ tc noderate-red $(5 R 4 / 6)$ and light-brownish-gray $\left(5 u_{R} 6 / 1\right)$, nodular

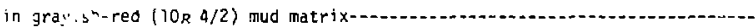
$587.0-58 B .5$

Nud, clay breccic, moderate-reddish-brown $\left(10_{R} 4 / 6\right)$ to dark-reddish-brown $\left(10_{2} 3 / 4\right)$. with soft, firm, moderate-reddish-brown $(10 R 4 / 6)$ silt containing numerous blebs and stringers of soft, crumbly, lightbluish-gray $\left(5_{B} 7 / 1\right)$ and medium-b]uish-gray $(595 / 7)$ silt and randomly dispersed, very finely and finely crystalline, colorless selenite; 1-4-cm-wide, soft, some chalky, subrounded to rounded, mocerate-reddish-orange $(10 R 6 / 6)$, moderate-red $(5 R 4 / 6)$, medium-1 ightgray (:6) and very 7 ight gray (18) gypsum nodules at 588.5-590.0 and 590.5-591.5 feet; argillaceous, saft, laminated to slightly mottled toriars base, gypsum band ai 590.0-590.5 feet, medium light gray (*6) and very light gray (N8) at top, moderate reddish orange $(10 R 6 / 6)$ and raderate reddish brown (10R $4 / 6)$

$\therefore u d / c l a y$ breccia, moderate-reddish-brown $(10 R 4 / 6)$ to dark-reddishbrown $\left(10_{R} 3 / 4\right)$, soft to fim; with numerous 1-5-cm-wide, chalky, soft, medium-3ight-gray (N6), white (N9), moderate-reddish-brown $(10 R 4 / 6)$ and maderate-reddish-orange $\left(10^{R} 6 / 6\right)$ to moderate-red $\{5 R 4 / 6\}$ gypsum nodules and some light-bluish-gray $(5 B 7 / 1)$ silt blebs; moderate-reddish-brown $(10 R 4 / 6)$ and some medium-light-gray (.6) gyjsum bands dipping $35^{\circ}$, very thin laminae of maderate-reddishbrown $\left(10_{F} 4 / 6\right)$ to dark-reddish-brown $(10 R 3 / 4)$ mud and mediumbivish-gray $(5 \mathrm{~B} 5 / 1)$ silt at 595.3 feec; numerous 8-mm-thick, parallel, fractured, white ( $N 9$ ) and medium-light-gray ( $N 6$ ) gypsum bands at 597.5-598.5 feet, dipping $35^{\circ}$ -

Gypsum, white $(x 9)$, chalky, soft, slightly fractured, some argillaceous;

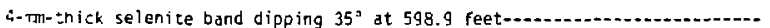

$598.5-599.0$ 
Tabie 3, - - bethologic log for borehole wrpp-25--Cont inued

Lithologic description $\quad \begin{gathered}\text { Depth interval } \\ \text { Feet }\end{gathered}$

Mud, grayish-red $(10 R 4 / 2)$ and moderate-reddish-brown $(10 R 4 / 6)$, soft to

finm, massive to crumbly, moderate-reddish-orange (10R 6/6) to moderate-

red $(5 R 4 ; 6)$ altered polynalite and $1-m-d i a m e t e r$ transiucent halite

crystals and 2-mm-thick halite stringers at 599.3-599.6 feet; madium-dark-

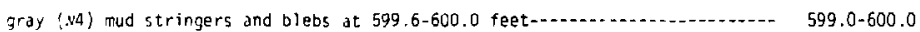

ratite, transiucent to traisparent, grayish-red (10R 4/2) tint, medium-crystalline,

slightly argillaceous, moderate-reddisin-orange $(10 R 6 / 6)$ poiyhalite

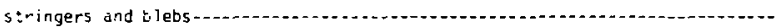

$600.0-6 C 2.5$

:10 core--...

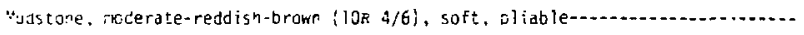

Hdite, translucent to transparent, pale-yellowish-orange $(10 \mathrm{R} 8 / 6)$ to

moderate-reddisn-orange ( $10 R$ 6/6) tint; many moderate-reddish-orange

$\{10 R 5 / 5)$ sclytalite stringers and blebs; 1-mm- to 2-cr-thick, wayy,

irreguiar, redium-light-gray $(: 66)$ and $1:$ aht-olive-gray $(5 Y 6 / 1)$

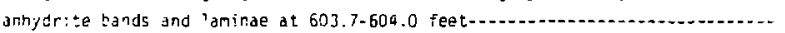

$602.5-603 . \mathrm{C}$

$603.0-603.1$

inhydri:e, rezim-! ight-gray $\{:, 6)$ and olive-gray $(5 ; 4 / 1), 1-m$-thick, wavy, irregsiar, light-olive-gray $\left(5_{5} 6 / 1\right)$ iaminae; very finely wistaline; contains numerous seamlets and ienses of colorless halice st 604.2-605.7 and 606.8-608.0 feet; a 70\%-dipping fracture at 608.1 faet: numerous 1 -mm-wide, irregular, generaliy linear vugs at 611.0 613.2 feet and 613.2-614.0 feet: thin wavy, irregular, moderatereddish-brown $\left(10_{R} 4 / 6\right)$ laminae at 613.2 feet; two irregular, horizontal 1-3-m-thick, halite-filled fractures at 614.4 and 614.5 feet, and numerous $4-15$-rim-diameter, rounded, soft, white $(w 9)$ biebs and vugs at 614.8-615.0 feet............

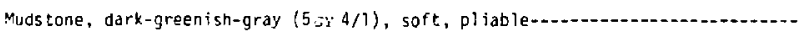

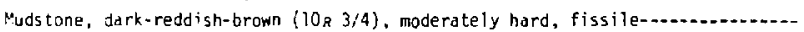

Halite, coiorless, medium-crystalline to coarsely crystalline; contains

many moderate-reddish-orange $\left(10_{R} 6 / 6\right)$ to moderate-reddish-brown

$\{10 R 4 / 6)$. White ( $N 9)$ and very light gray ( $\mathrm{NB}$ ) polyhalite stringers

and slebs: argillaceous, especially at 617.3-ot9.5 feet-

$604.0-675.0$

$675.0-676.0$

$616.0-616.4$

Halite, colorless, Firely to ineciula-crystalline, minor moderate-

recdisn-orange (:0P E/6) to roderate-reddish-brown (108 4/6)

wolyhalite strirgers and blebs: white (N9) to very light gray ( $* 8$ )

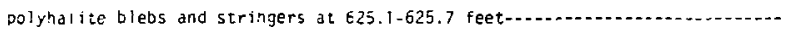

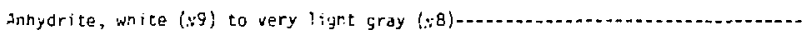

tali:e, colorless, finely to medium-crystalline, nume rous moderate-

reddish-crange $(10, E ; 6)$ polyhalite stringers and blets

$623.2-627.6$

$627.6-628.3$

$628.3-631.4$ 
Table 3.--tithologic log for borehole WIPP-25--Continued

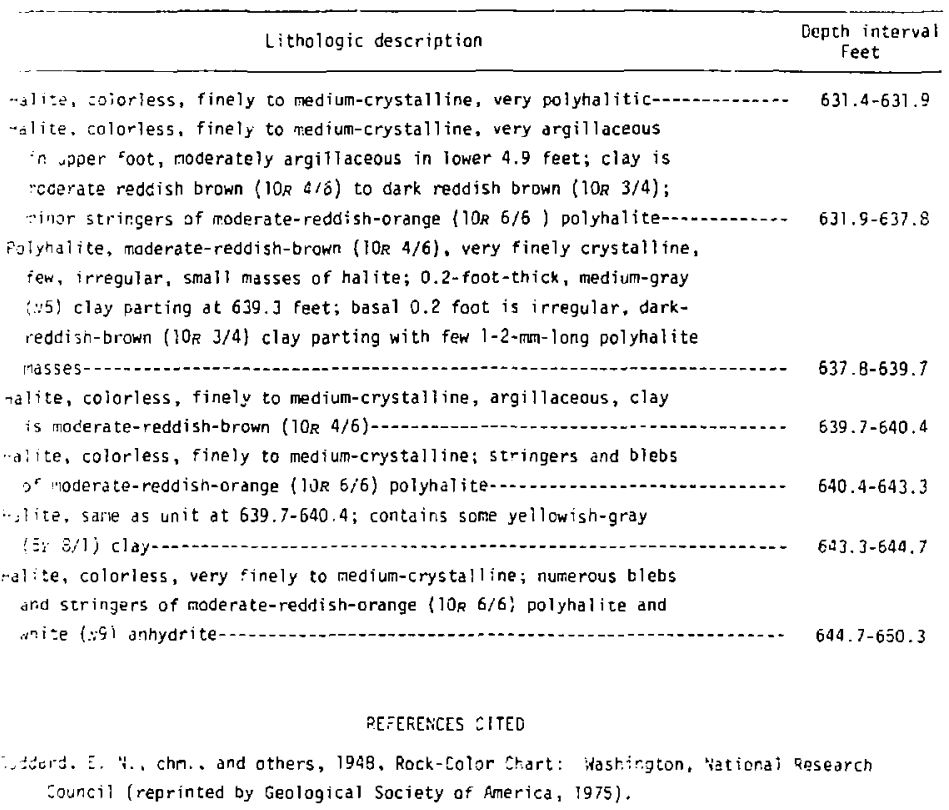




\section{6 . BIBLIOGRAPHY}

Bachnan, G. O., 1974, Geologic processes and Cenozoic history related to salt dissolution in southeastern New Mexico: U.S. Geol. Survey Open-Eile report $79-194$.

Bachman, G. O., 1975, Cenozoie deposits of southeastern New Mexico and an outline of the history of evaporite dissolution: U.S. Geol. Survey, Jour. Research, v. 4, no. 2, p. 135-i49.

Bachman, G. O., and Johnson, R., 1973, Stability of salc in the Permian Salt Basin of Kansas, Oklahoma, Texas, and New Mexico: U.S. Geol. Survey Open-file report $4339-4$.

Godidard, E. N., Chm., and others, 1948, Rock-Culor Chart: Washington, National Research Council (reprinted by Geological Society of Amer ica, 1975).

Jones, 6. L., 1973, Salt deposits of Los Medanos area, Eddy ar.d Lea Counties, New Mexico, with sections on Ground-water hydrology, by M. E. Cooley and Surficial geology, by G, O. Bachman: U.S. Geol. Survey Open-E 1 le report (USGS-4339-7), 67 D.

piper, A. M., 1973, Subrosion in and about the four-township area near Carlsbad, New Mexico: ORNL subcontract 3745, Oak Ridge National Laborator $Y$, Oak Ridge, TN.

Powets, D. W., Lambert, S. J., Shaffer, S-E., Hill, L. R., and Weart, W. D., editors, 1978, Geological Characterization Report, Waste Isolation Dilot Plant (WIPP) Site, Southeastern New Mexico: Vol. I \& II, SAND78-1596, Sandia Lahorátories, Albuquerque, NM.

Vine, J. D., 1963, Surface geology of the Nash Draw quadrangle, Eddy County, New Mexico: U.S. Geol. Survey Bull. 1141-B. 
APPENDIX A

JUST IFICATION

by

S. J. Lambert

Division 4511

Sandia Laboratories 
INTRODUCTION TO APPENDIX A, JUSTIFICATION

Appendix A consists of relevant portions of two related documents:

1) memorandum from 5. J. Lambert to Distribution, dated $6 / 8 / 78$, "hIPP programs to Investigate the Nature and Eifects of Salt Dissolution and Overburden subsidence in the Vicinity of the WIPP site," and

2) memor andum Erom S. J. Lambert to R. D. Statler, dated 6/29/78, "Criteria for WIPP 25 Through WIPP 30, and Their Quality Assurance."

These documents provide details of background information and program options as understood at the tine of initiation. The reader is cautioned, therefore, that details of the program may have been altered as information became available and that geliminary interpretive hypotheses or ideas guiding the program formulation may need revision based on information presented in this report. Later interpretive reports may deal with such items. 
date June 8,1978

Sandia Lalıratories

to Distribution

Alouquerque Nevr Mexco

Livermare Calsforna

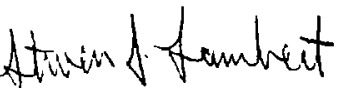

$1 \mathrm{rmm}$ S. I. Lambert - 5311

suhe: WIPP Programs to Investigate the Nature and Effects of Sal, Dissolution and Overburden Subsidence in the Vicinity of the WIPP site

\section{Definitions of Problems}

Bedded deposits of Permian rock salt of the Ochoan epoch in southeastern New Mexico's pcition of the Delaware Basin are not found near the surface because of halite's appreciable solubility. These deposits are preserved at depth where the overburden rocks' permeability is low enough to preclude the exposure of rock salt to significant amounts of circulatinc. solutions which are not saturated with sodium chloride. when the overburden barrier is somehow breached, fluids may more easily reach the salt. The salt dissolves, is removed, and the overburden collapses into the void space left as a result of salt removal. Commonly this collapse is expressed at the surface as a set of features such as sinkholes, and a karst topography reminiscent of limestone terrain develops. Since the most prevalent soluble rocks of the Delaware Basin are halite and anhydrite, not limestone, the relationship between salt dissolution and subsequent development of karst-like surface features is not altogether well defined. Whereas limestone is but sparingly soluble in meteorically-derived water, and spectacular sinkholes may be observed at the surface in some localities, sinkholes which might develop in salt at depth are obscured by the overburden, which has since collapred into comonly chaotic rubble, particularly near the surface. In addition, limestone has greater mechanical strength than rock salt, and has greater potential of maintairing an open sinkhole than salt, which has a tendency iu self-seal under overburden pressure. Gypsum (formed from the oricinal anhydrite by interaction with near-surface groundwaters) is intermediate in solubility between limestone and rock salt. and might more readily maintain a cavity than would rock salt.

An additional complication encountered in studying karstic features in southeastern New Mexico is the climate. The sparse vegetative cover in semiaria karstic regions leads to minimal soil stabilization; many small scale features indicative of subsurface dissolution of rock and collapse of overburden are filled in with poorly consolidated surface material almost as soon as they are formed. This appears to be the case of surface 
fractures associated with subsidence over mines and cataclysmic collapse episodes (such as occurred at San Simon Sink in historic times). These fractures were quickly filled by blowing sand.

A primary concern for a radioactive waste repository in bedded salt is long-term integrity of the salt itself. In the case of the WIPP site at Los Medanos, a zone of apparently active dissolution of salt has been identified near the top of the Salado Formation (about 1300 feet or 400 meters above the uppermost horizon proposed for waste emplacement) at the western periphery of the proposed 30-square-mile land withdrawal area. Whereas no small-scale karstic features are developed in this area, and evidence for dissolution in the area is based entirely upon subsurface borehole data, a large depression called Nash Draw is developed to the west of the area. Exploratory drilling for potash in Nash Draw has revealed the removal of halite in the Rustler and Salado Formations and the development of a permeable brine-bearing zone of residue of dissolved evaporites called the "brine aquifer". The brine aquifer was probably encountered in hole P14 on the western margin of the WIPP area, yet the actual topographic edge of Nash Draw is no closer than one mile west of the WIPP land withdrawal area. Thus the development of Nash Draw is shrouded in mystery: what has been the relationship between dissolution of salt and subsidence of overburden in Nash Draw?

What are the implications (regarding WIPP-horizon salt integrity) of fluid movement, dissolution and subsidence involving salt above the WIPP? And how do these implications relate to safety assessment of WIPP through scenarios involving the supra-salado rocks at the WIPP site and the ability to carry radionuclides in their aquifers into the biosphere? G. Bachman in USGS OpenFile report 34-194 (1974) estimated average rates of salt dissolution in this area of about six to eigin miles per million years horizontally, and $1 / 3$ foot per thousand years vertically. If these estimates are correct, further evaluation of Nash Draw is unnecessary; it would, however, provide confidence in the earlier estimates.

An additional concern is the region that must be controlled around the WIPP. Currently, DOE prescriptions specify control of a two-mile radius around the possible WIPP workings. Within this zone, various degrees of resource production will be allowed. If the effects of subsidence were understood and of lesser consequences than allowed for today, greater production of potash and hydrocarbons might be acceptable within portions of the two-mile zone. 


\section{Program Objectives}

The program of investigation of dissolution and subsidence has four fundamental purposes, with one special-case consideration:

1. To correlate surficial collapse features and deposits with subsurface dissolution, in order to develop criteria for determining if there is an evolutionary pattern of collapse.

2. To characterize subsurface dissolution products adjacent to the WIPP site.

3. To determine the behavior of fluids in dissolution products adjacent to the WIPP site.

4. To analyze potential impacts of evolution of dissolution products at and near the WIPP site with respect to repository breachment and radionuclide transport.

The special case is to determine the nature of subsidence over mines in salt, and its effects on the overlying groundwater system.

An elaboration of these purposes follows.

\section{Nash Draw Investigations}

Nash Draw, the "dog-bone" shaped depression west of the WIPP site, is thought to have originated by some combination of surface erosion and subsidence following subsurface dissolution. If the process of formation is overwhelmingly erosion, then the potential for removal of overburden at the WIPP site is probably about the same as it has been in Nash Draw. If, however, the process is overwhelmingly dissolution of salt and collapse of overburden, the potential exists for the development of a complex system of stratigraphic and hydrological relationships over the WIPP, much as what has developed in Nash Draw. At present, there is no conclusive way of defining an instantaneous rate of growth

of Nash Draw toward the WIPP site; therefore, the only alternative is to understand the processes which have resulted in Nash Draw, and incorporate their implications into the mathematical modeling efforts directed toward safety assessment involving the WIPP site in general, and radionuclide escape and migration in particular.

First, the Nash Draw program is a series of core holes, which will be geophysically logged. This operation will obtain data to serve three purposes: 1) reveal the subsurface Nash Draw stratigraphy at carefully chosen points, 2) reveal the relationships between the subsurface structure and surface features and deposits, 3) reveal how much halite and anhydrite (or gypsum) has been removed by dissolution. The holes will indeed be 
located near surface features which sugges' that dissolution and collapse in the subsurface has taken place, so that the relationships between surface and subsurface can be verified (item \#2). Stratigraphy of post-Nash Draw deposits will help to unravel the history of development of the depression ( $\# 1$ above). Examination for missing evaporites will identify how much dissolution has taken place to produce the observed surface features, and where dissolution has taken place to various degrees (\$3). Characterization of surface features found at sites having experienced various degrees of dissolution will allow for the determinatior of the pattern of evolutionary collapse, if any. Thus, the fulfilling of these three data requirements should satisfy the spizit of program objective \#1.

Second, the Nash Draw program is a source of geologic material for petrographic and geochemical examination. Making thin sections of recovered rock will allow the mineralogy of aissolution products to be determined, i.e. identification of what was dissolved and what remains. Mineralogy and textures of dissolution residues and cemented collapse fill can then be compared with those of dissolution features sampled in other programs, such as the one to investigate the cemented rubble chimneys (oft called "karstic domal features" or, incorrectly, "breccia pipes"). Comparisons can be made to see if Nash Draw and cementeo rubble chimneys have involved similar processes of formation. Geochemical analyses of core materials for trace constituents will reveal their degree of interaction with groundwaters, and possibly an age of formation. Alsn, permeabilities toward fluids can be obtained on cores. Thus, the second program objective is satisfied.

Third, the Nash Draw program will become a series of hydrology holes. The "brine aquifer' underlying Nash Draw has been attributed responsibility fcr much of the dissolution and collapse observed today. The same "brine aquifer" has been attributed responsibility for carrying radionuclides should they: escape from WIPP to the biosphere, allegedly via the seeps at Malaga. It therefore behooves is to understand the potentiometric patterns, permeability variations and fluid quantities found in that aquifer. Furthermore, the safety assessment scenarios for radionuclide escape and migration involve movement from WIPP into Rustler Formation waters into the "brine aquifer" and out at Malaga. It also behooves us to understand the hydrologic relationships between the "brine aquifer" and the Rustler water-bearing rocks in Nash Draw. And with this understanding, frogram objective $\$ 3$ is satisfied. 
For fiscal year 1978, four and possibly five Nash Draw holes have been proposed. It is expected that the program will extend through the fiscal year 1979, with up to nine additional holes, logistic resources permitting. The first six holes are located and identified according to nearest named landmark as follows:

$\begin{array}{ll}\text { WIPP-ND-1 } & \text { SI5-T22S-R30E } \\ \text { WIPP-ND-2 } & \text { S29-T22S-R30E } \\ \text { WIPP-ND-3 } & \text { S21-T21S-R30E } \\ \text { WIPP-ND-4 } & \text { SI8-T21S-R3IE } \\ \text { WIPP-ND-5 } & \text { S35-T22S-R29E } \\ \text { WIPP-ND-6 } & \text { S32-T21S-R31E }\end{array}$

Crawford Ranch

Fortyniner Ridge

Red Lake

New Well

Tamarisk Flat Noye Tank

Surface casing (conductor pipe) is to be set similar to WIPP 19. Holes 1,2 and 4 , are to be cored as completely as possible into the upper salado Formation to an identifiable marker bed. The core size should be $35 / 8$ nominally, taken to TD. Approximate predicted TD's for the first four holes are 500, 350-400, 400 and 500-600 ft, respectively. A geologic prognosis of each location will be provided by $C$. I. Jones (USGS) prior to drilling each hole. In addition, resistivity surveying in Nash Draw will precede drilling, if possible. Loss of circulation is anticipated in holes 1,2 and 3 .

Geophysical logs in the holes should include: resistivity, density, garna, neutron, sidescan, and caliper. The sonic log might not be obtainable if the hole cannot maintain a fluid column long enough, but is desirable. up-hole velocity to aid seismic interpretation might be appropriate.

After the drilling (and/or coring) and logging, it is planned to stand $51 / 2$ inch casing and cement it to the surface. Each hole then becomes available for hydrologic testing and monitoring. Each l:oie will then be subjected to the same sorts of treatment as described in SAND 77-1401. Information so obtained includes piezometric potentials, permeabilities, quantities of Eluids and the degree of connectedness between the Rustler rocks and the "brine aquifer." In additior, water samples will be analyzed to determine their origins and role in dissolution. The cores of water-bearing rocks can be analyzed for porosity and nuclide-sorption affinit;. The results can be implemented in modeling efforts for safety assessment, and program objective \$ 4 will have been satisfied.

As well as modeling groundwater movement, it may be advantageous to attempt to model the dissolution that might govern the future development of Nash Draw, once the evolutionary realtionships among processes in the cycle: dissolution - collapse fracturing - water entry - dissolution, are understood. 


\section{Cemented Rubble Chimmey Investigations}

one of the "karstic domal features" described by J. D. Vine (called Hill "C") was encountered at the level of the McNutt potash zone by Mississippi Chemical Corporation. It was found to be a chimney in the salado Formation filled with claycemented brecciated rock belonging to strata above the Mclutt. Similarly a breccia-filled chimney was encountered in drilling mar a circular hill near the Weaver Mine. There are numerous other eroision-breached domes such as Vine's llill "C" in the vicinity of Nash Draw; the subsurface expression of them, if any, is virtually unknown. Recent geophysical surveys of the region have revealed that many of these domes, including the Wellsweaver and Hill "C", are associated with resistivity lows.

One such resistivity low, without conspicuous surface expression, has been reported in section $17, T 22 S, R 31 E$, inside the WIPF area. This suggests a localized increase in fluid content of rock and possibly a local porosity high and calls for an evaluation of the resistivity low as a possible fluid-carrying avenue. Implications of fluid movement in evaporites are, of course, dissolution and collapse. once the initial resistivity survey and location of the section 17 low is corroborated by a second survey, WIPP borehole \#13 will be drilled to determine the nature of the low. Should the low prove to be a rubble chimney near the surface, a decision point is defined, requiring 1) careful investigation of the nature and properties of the section 17 feature including a deep exploratory hole, WIPF 16 , to determine the threat, if any, or 2) temporary abandonment of WIFP 13 while WIPP 16 is drilled in another resistivity low, which might have a breached dome, or even in one of the known cemented rubble chimneys, or 3) site abandonment if the implications regarding threat of such a feature to HIPP safety are never to be understood.

Should a decision be necessary, the most scientifically expedient yet intellectually conservative approach is option $\$ 2$. Experience in driling (ano perhaps instrumentation development) yaised from WIPP 16 in a "known" (or at least strongly suspected) rutple chimney will be welcome when the onsite resistivity iow is revisited to investigate it in detail.

A procram for WIPP 16 in the case of a "kncwn" chimney shoula consider the following information requirements:

1. Recovery of core from as many portions of the structure as possible.

2. Recovery of representative analyzeable samples of any liguids encountered. 
3. Recovery of representative analyzeable samples of any gases encountered.

4. The option to make in situ determinations of permeabilities to liquids and diffusion coefficients for gases.

5. Determination of the reservoir size, if any, of liquids and gases.

6. As complete a description of the physical, chemical and geological properties of the structure as is practicably obtainable.

The achievement of these goals will probably require the following considerations:

1. Use of a drill rig able to penetrate about 5000 feet of section.

2. Employment of blowout preventers, fluid detection, alarm and collection systems.

3. The option of drilling with air or mud.

4. The ability to take 4 -inch core by the rotary method from top to bottom insofar as practicable.

5. The option of whipstocking off a central (axial ?) hole, to take radial cores.

6. Directional surveys to allow "mapping" the subsurface extent of the structure.

7. The taking of geophysical logs, including resistivity, density, gamna, neutron, sidescan and caliper; sonic if possible.

8. The option to multiply complete the resulting hole(s) for hydrologic testing.

It is desirable that this hole be drilled to a depth at which no more brecciated rock is found. Should solid rock be encountered in the reef complex, it is recommended that the hole be cased down to the carbonate for hydrologic observation. Hydrologic observation (including water sampling and piezometry) in fluid-bearing zones of the structure itself and in "becrock" and in nearby rocks will help to determine the degree of connectedness of the structure with aquifers of regional extent. 
Petrographic analyses of ccre recovered will allow comparisons to be made with dissolution products recovered from Nash Draw investigations, described previously. Such material also lends itself to geochemical analyses for the determination of rockwater interaction history and age. Similarly, fluid analyses will provide an indication of how the fluids have interacted with their host rocks, helping to determine the processes at work in such structures. The rock geochemistry from WIPP 16 car also be compared with Hill "Cr" sampled in MCC mine.

Since the modeling of radionuclioe escape and migration must take into account fluid movement, cemented rubble chimneys should be evaluated for the potential as a path for such movement. They should also be evaluated as members of the evolutionary pattern of dissolution and collapse features, and might be generically related to the development of Nash Draw. Consequently, it is desirable to measure in situ the permeability of the structure. WIPP 16 could provioe the opportunity for development of necessary instrumentation to be used in the exploration of the WIPP 13 feature. In additicn, the hydrologic relationships between the chimney and surrounding rocks should be understood in order to formulate a complete hydrologic model for risk assessment. Thus, the four program objectives will have been met for the case of cemeiiled rubble chimneys and these dissolution products can then be related to those encountered in the Nash Draw program. 


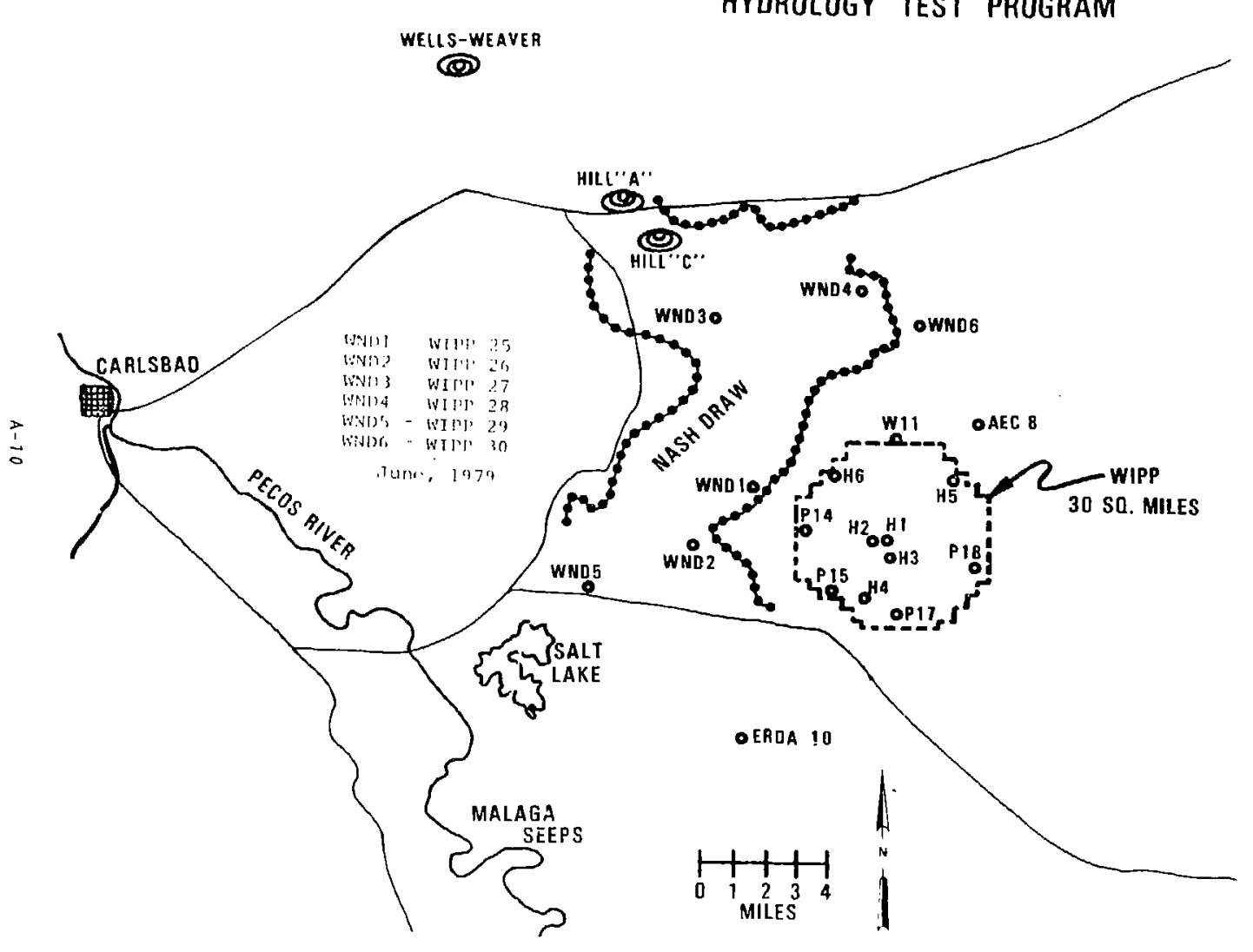


to R. D. Statler, 1133
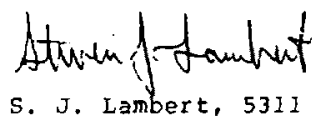

sut!e: : Criteria for WIPP 25 Through WIPP 30, and Their Quality Assurance

Reference: S. J. Lambert to Distribution, dtd. 6/8/78, "WIPP Programs to Investigate the Nature and Effects of Sa] $t$ Dissolution and Overburden subsidence in the Vicinity of the wIPP site"

Purposes

The holes WIPP 25 through WIPP 30 are informally known as NDI through ND6.

First, these are a series of core holes to be geophysicaliy logged, to serve three purposes: (l) reveal the subsurface Nash Draw stratigraphy at carefully chosen points, (2) reveal the relationships between the subsurface structure and surface features and deposits, (3) reveal how much halite and anhydrite (or gypsum) has been removed by dissolution.

Second, these holes are a source of natural geologic material for petrographic and geochemica? examination of products of djssolution of evaporites adiacent to the WIPP area, for studies of age-dating. rock-water interactions and related phenomena.

Third, the Nash Draw holes are to become a series of hydrology holes to provide the modelling (safety assessment) effort with data regarding dissolution and groundwater flow in a geological!y complex piece of territory, between the west borde of the WIPD area (Livingston Ridge) and the Pecos Rive: , the nearest permanent surface water aczessible to humans.

\section{Locations}

The latera) location of each hole was selected by the water Resources Division of the United states Geological Survey. The precise location of each hole is governed by the surface feature it is designed to explore. In general, a latera] 
Jocation tolerance less than $+1 / 2$ mile is suffjcjent, but some features of interest might méasure less than $1 / 2 \mathrm{mile}$ in any surface dimension, and, therefore, the hole must fa: 1 within the feature of interest. The as-built location of each hole is to be surveyed after completion of all operations so as to be known within one foot with respect to existing section boundaries, usually the nearest marked section corner.

The approximate hole locations are given in the document referenced above.

Surface Elevations

A datum point at the surface at each hole Jocation should be established to within + one foot vertically with respect to known elevations nearby, usually the nearest Nationaj Geodetic survey first-order levelling survey markers, to serve as a reference for depths measured in each hole. A recoverable survey marker should be emp? aced at the surface near each hole to ifientify the lateral and vertical co-ordinates of this datum with respect to geographic co-ordinates and to the Jocation of the hole itself.

Dr 1 ling and Coring

Continuous core is to be taken in each hole from surface to total depth. The core should be at least $3-5 / 8$ inches in diameter, or the largest practically obtainable with a shal? ow-hole $r$ ig. Anticipated total. depths are those described in the retter of C. L. Jones (USGS) to P. D. Seward (SLA-1135) dater Tune 7, 1978, It is permissible to drilj with mud gel, but circulation might be Jost so appropriate additives to the mud are warranted. Completion of ajl holes as hycrology holes is anticipated for water sampling and measurements of prezometric elevations and permeabilities.

Fu'? core recovery (with origin depths documented to within tone foot) is highly desirabje to facilitate thorough

investigation of rocks associated with evaporite dissolution in Nash Draw. However, the exploratory nature of this program takes account of the fact that geological condjtions in these holes are imprecise?y known, and might not be conducive to ful? core recovery. In this case, core obtained should be docimenter according tc actuat percentage of recovery, and origin depths of recovered core should be approximately determined through combinations of other techniques, including geophysica? logs and comparison with stratigraphically equivalent cores from other ho'es.

Core should be handled in accordance with estahlished sandia procedures. These genera' procedures have been given in the 
letter to distribution from R. D. Statler (dtd.-5/15/78) containing the field operatiors program for WIPP holes $H 4 a$, $b$, and $\mathrm{c}$.

\section{Geophysical Logging}

Three kinds of information are to be obtained by geophysical. * ogging: (1) maintenance of stratigraphic control, Identification of rock types in the hole and verification of core depths; (2) identification of fluid-bearing zones and. qualitative evaluation of relative permeabilities for future hydrologic investigations; (3) measurement of up-hole sound-wave velocity, to determine elastic properties of the rock to aid in the interpretation of future seismic surveys of Nash Draw, if any. For example, the first kind of information might entail density, natural gamma, neutron and sonic logs. The second kind might entail $R_{x o}, R_{t}$ and neutron logs. The third kind might entail sonic and up-hole velocity logs.

\section{Casing}

A].1 the holes are to be cased to about 50 feet below the top of salt (past the evaporite residuum at the top of the salado Formation), using $5-1 / 2$ inches (minimum) outside diameter steel casing, to allow for multiple completion for hydrologic testing at a future time.

\section{Cementing}

Cementing of casing is to be accomplished so as to isolate aquifers from one another so that fluids from them do not cross-flow through the cement. Bond logs and tracer testing will be prescribed later during the hydrologic testing program, at which time the integrity of the cement behind casing will be evaluated.

Final Operation

After casing and cementing, the holes are to be filled with fresh water and temporarily abandoned, awaiting hydrologic testing.

SJL: $5311: \mathrm{rmf}$

Approved :

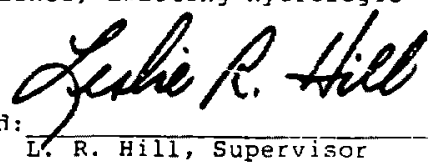

Nuclear Waste Technology

Division 5311 
APQENDIX B

DRTLLING AND TESTING PLAN

by

R. D. Statler

Divisjon 1133

and

P. D. Seward

Division 1135

Sandia Laburatories 
INTRODUC':ION TO APPENDIX B, DRILLING AND TESTING PLAN

The drilling and testing plan is the translation of technical objectives contained in documents in Appendix A into field engineering terms. Changes or amendments are included as well. The approvals and permits obtained from various agencies prior to drilling are kept on file but are not included here. 
Field Operations Program of Sandia Labs

WIPP site Investigations

(Nature Effects of Salt Dissolution, Nash Draw)

Exploratory Wells :

$\begin{array}{ll}\text { WIPP } & 25 \\ \text { WIPP } & 26 \\ \text { WIPP } & 27 \\ \text { WIPP } & 28 \\ \text { WIPP } 29 \\ \text { WIPP } 30\end{array}$

Nash Draw Locations

Sections:

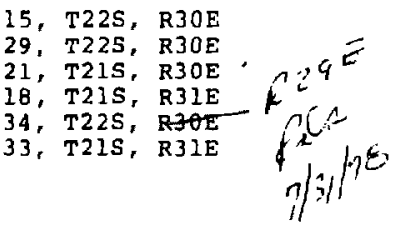

Purpose: To reveal subsurface stratigraphy and hydrologic setting of Nash Draw in order to determine the relation between structures, surface features and evaporate dissolutioning.

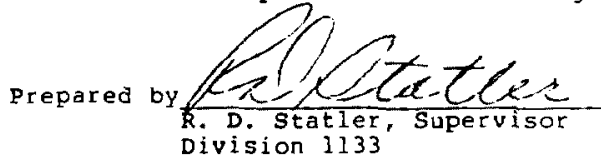
Division 1133

Field Engineering Projecfs

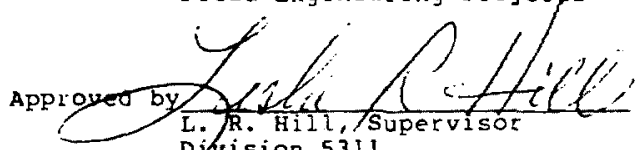

Djoision 5311

Puclear waste Technology

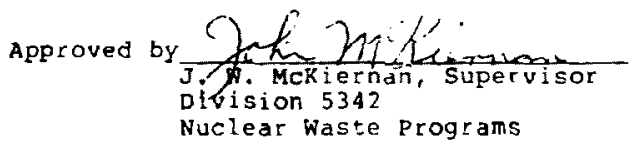


Au: July 26,1978

Distribution

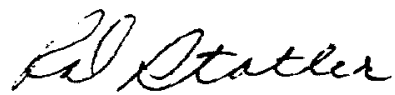

\section{Sandia Laboratories}

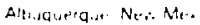

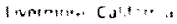

R. D. Statler -1133

Nash Draw Exploratory Wells, WIPP 25 through 30

The attached document contains the Fleld operations plan for conducting Wipp Site Investigations in the Nash Draw. Drilling, logging, coring, and cementing procedures are included for your use and information, Revisions and additions shall be added to this document as required and appropriate distribution made. $\operatorname{RDS}: \mathbf{r} \mathbf{j}$

Distribution:

C. L. Jones, USGS, Specfal Projects Division, Denver, CO

$J$. W. Mercer, USGS/WRD, Albuquerque, NM

G, o. Bachman, USGS/WRD, Albuquerque, MM

$R$. Taft, DOE/NVOO, Las Vegas, NV

D. Schueler, DOE/ALO

J. Cross, F\&S, Las Vegas, NV

W. E. Cunningham, F\&S, Carlsbad (3)

1130 H. E. Viney

2133 C. W. Gulick

1135 P. D. Seward

1135 J. E. Magruder

5310 Archives (2)

5311 L. R. Hill

5311 S. J. Lambert

5311 D. W. Powers

5312 H. C. Walker

5342 J. W. McKternan

5342 R. Hogan

9517 F, L. McFarling (2)

1133 R. D. Sratler (3) 
TABLE OF CONTENTS

Page

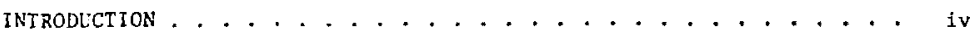

I. FIELD OPERATIONS PRUGRAM CRITERIA . . . . . . . . . . . . . 1

II. FIELD OPERATIONS PLAN

A. Organization...................... 4

B. Supporting Data . . . . . . . . . . . . . . . . 5

C. Drilling Parameters and Expected Drilling Requirements . . . . 9

D. Discussion of Potential Hazards . . . . . . . . . . . . 10

III. FIELD JPERATING PROCEDURES FOR QLIALITI CONTROL REQUIREMENTS

A. Measurement of Surface Location and Elevation . . . . . . . 12

B. Core Logging and Handling and Storage . . . . . . . . . . 13

C. Geophysical logging . . . . . . . . . . . . . . . . . . 14

D. Casing and Cementing. . . . . . . . . . . . . . 18

II. REPORTS . . . . . . . . . . . . . . . . . . 19 


\section{INTRODUCTION}

This document contains design eriteria for $61 x$ exploratory wells to investigate stratigraphy and hydrology in the Nash Draw. It describes the operational plan for conducting the fleld activities required to teet specified objertives. It includes procedures with drawings, specifications, and instructions necessary for good quality control of essential features. The Sandia Quality Assurance Program Plan for WIPP Site Evaluation is expected to cover activities associated with this field program. 
I. FIELD OPERATIONS PROGRAM CRITERLA

The following memorandum has been accepted an the degign criteria for the WIPP Stte Investigations of the Naoh Drạ, therefore, It 18 being reproduced in its encirety for use in the conduct of this project.

date June 29,1978

to R. D. Statler, 1133

trom. S. J. Lambert, 5311

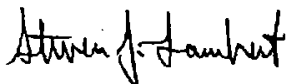

\section{Sandia Laboratories}

Albuquerqua. Now Mexico Livermore. Calitornis

subject Criteria for WIPP 25 Through wrPP 30 , and Thelr quality Assurance

This memor andur is separately zeproduced in Appendix $A$. Pages $1-3$ of the drilling and testing program are therefore abridged. 


\section{FIELD OPERATIONS PLAN}

\section{A. Organdzation}

Technical direction will originate within Sandla Division 5311. Field operations, wanaged by Bob Statler, Sandla Division 1133, w111 be conducted by W. E. Cunninghan, Fenix \& Scisson. Drilling contract and assoclated support service contracts will be let and administered by F\&S as arranged for by Federal Agency order through Nevada Operations office, DOE.

Identification of marker beds, core logging and other geologic interpretations will be provided by duty geologist.

Qualit; control and inspection wll be conducted by designated experts. Quality assurance program will be administered by F. L. McFarling and Jim Jones, Sandia Division 9517.

Industrial Safety Program will be administered by specialists from Fenix \& Scisson, Las Vegas.

Administrative assistance, logistical support of Sandia Programs will oe provided by P. D. Seward and J. E. Magruder, Sandia Division 1135. 
B. Supporting Data

Ceolog:

The data for the following chart was provided by Charles L. Jones, Geologist, USGS, Special Projects Division, Denver, CO, in a letter from Jones to $P$. D. Seward, 1135, ded $6 / 7 / 78$.

"Geologic Prognosis for Nash Draw Boreholes" (Depth Intervals in Feet)

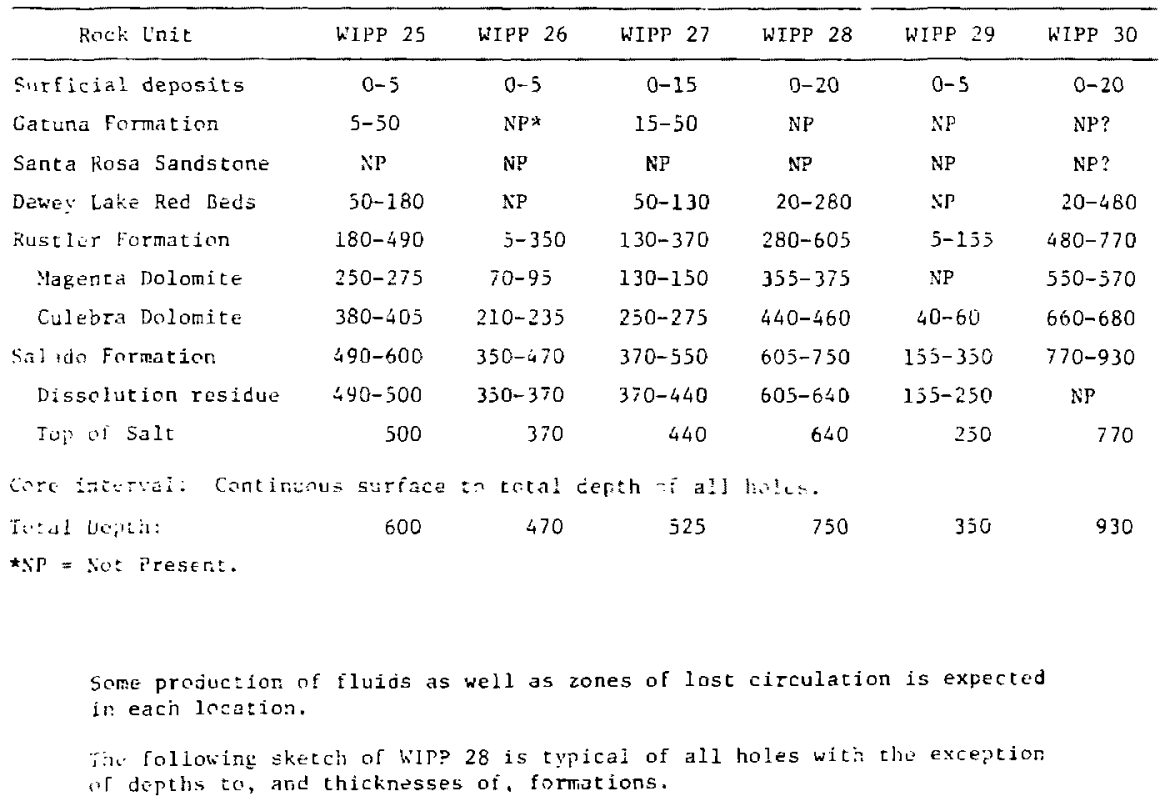




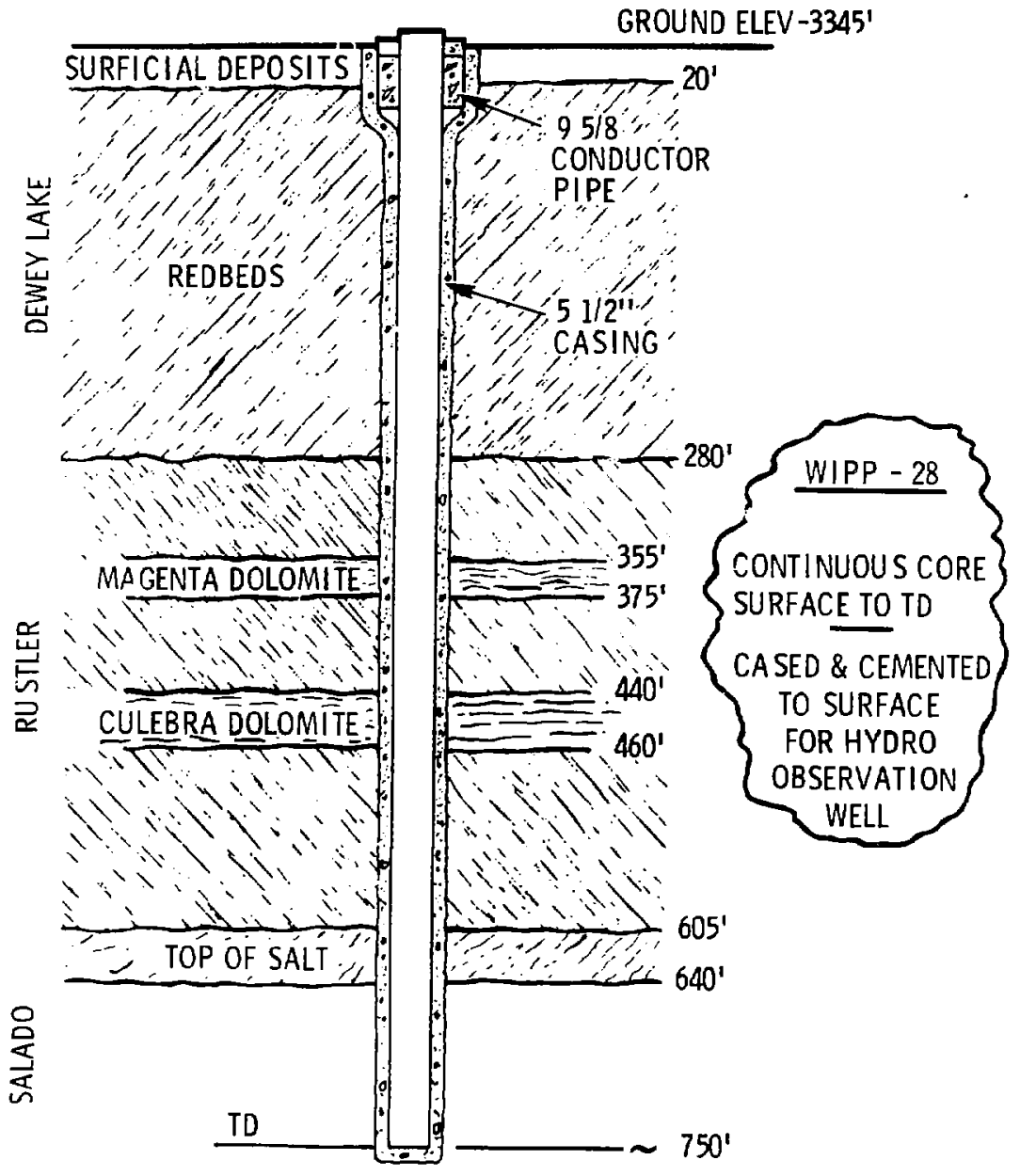


NLW MrXico

NASH DRAW OUADRANGLE 15-MINUTE SEATES

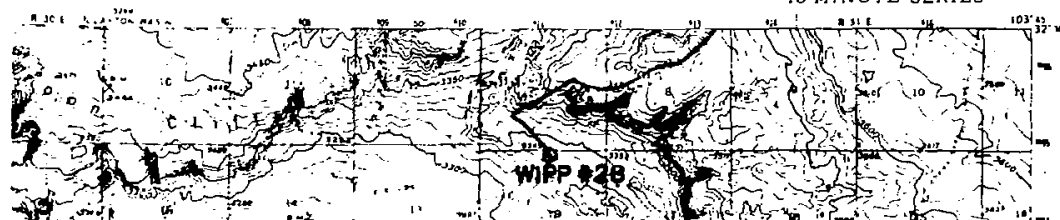

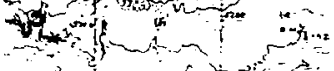

$\therefore$ and

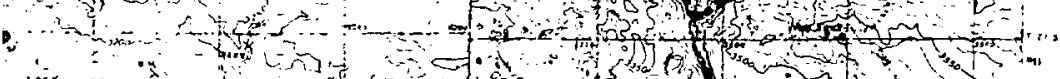
$\therefore$ : * " 4..

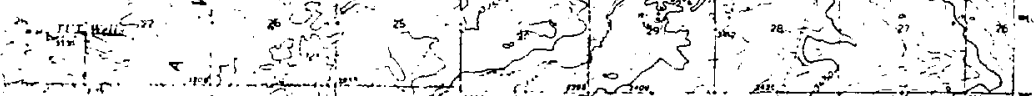

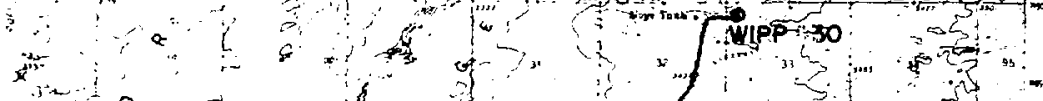

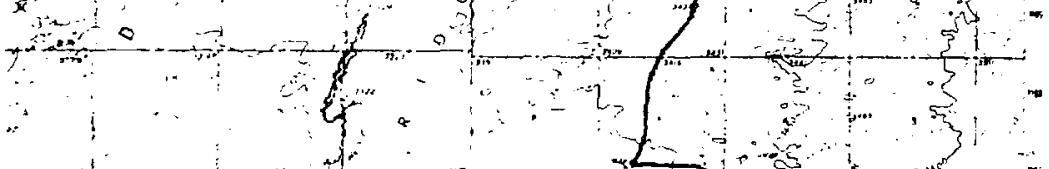

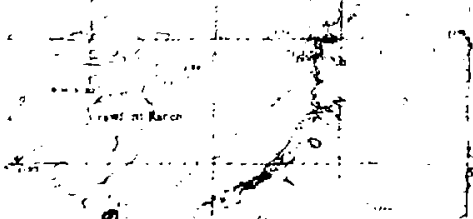
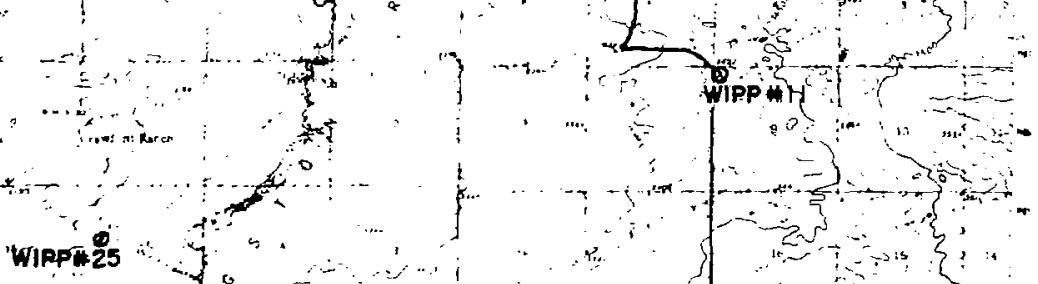

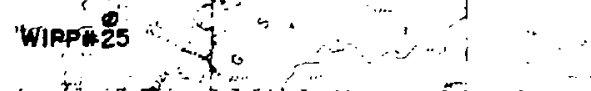
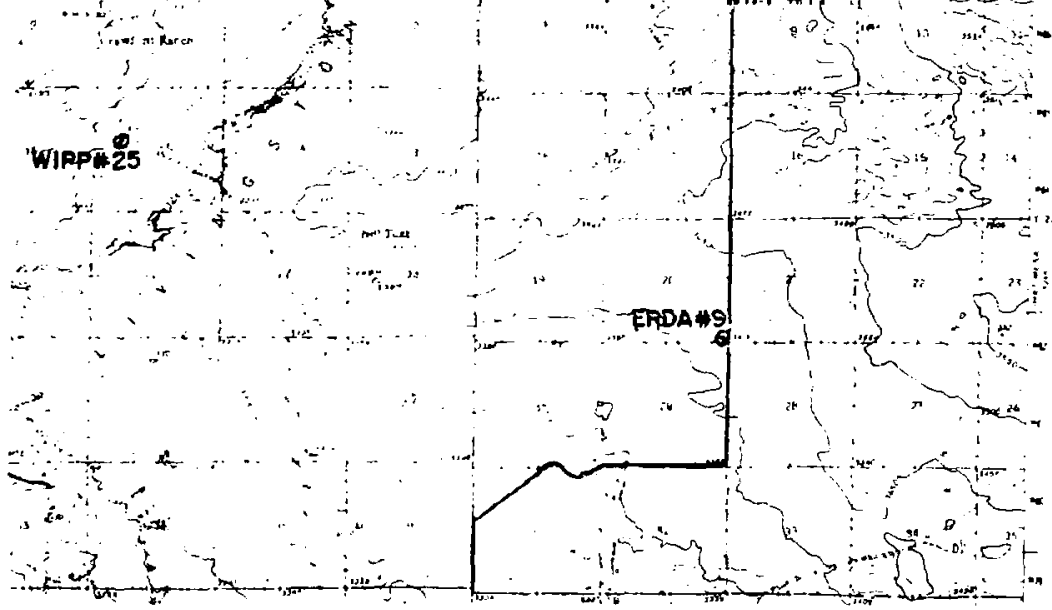
$\because$ DEPARTMEITED STATES

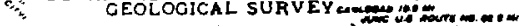
23:

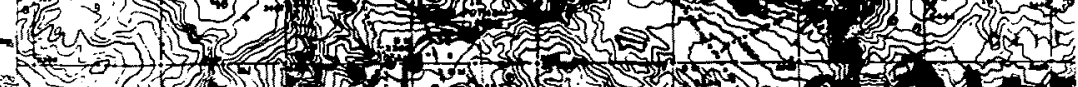
in (2) (1)

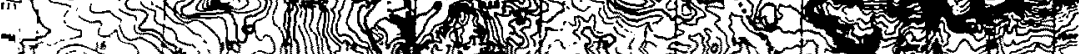

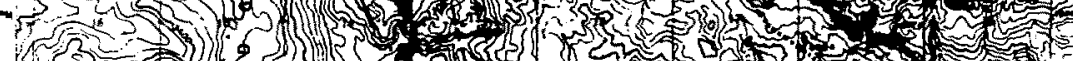

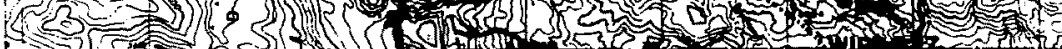

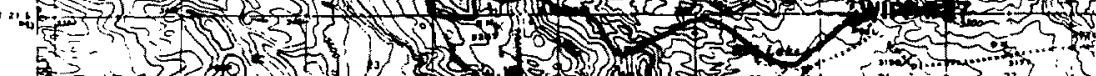
5, [.

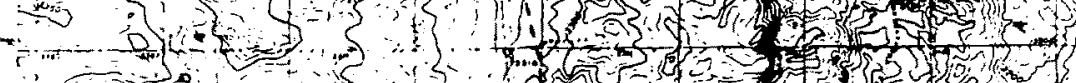

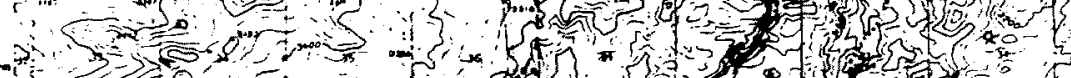
5, 5 ?

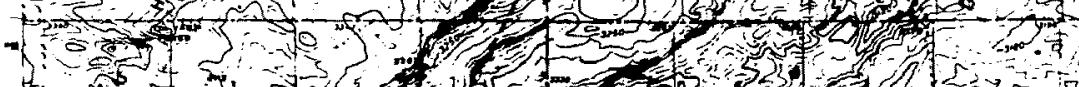
F.

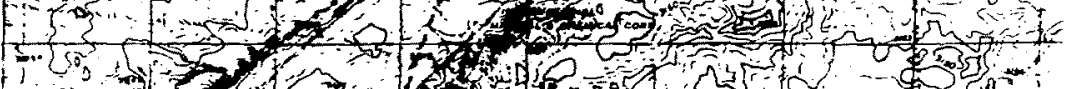

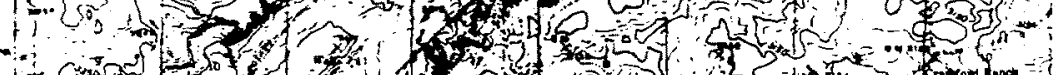
$10\left\{\begin{array}{l}0 \\ 0\end{array}\right.$

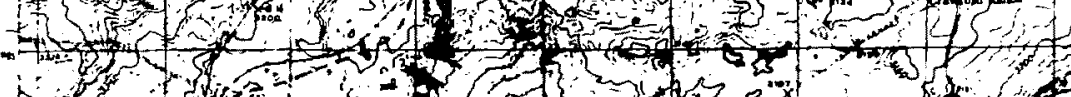

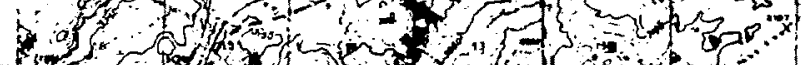

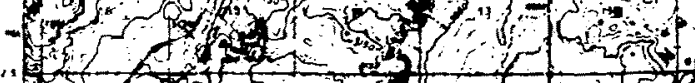

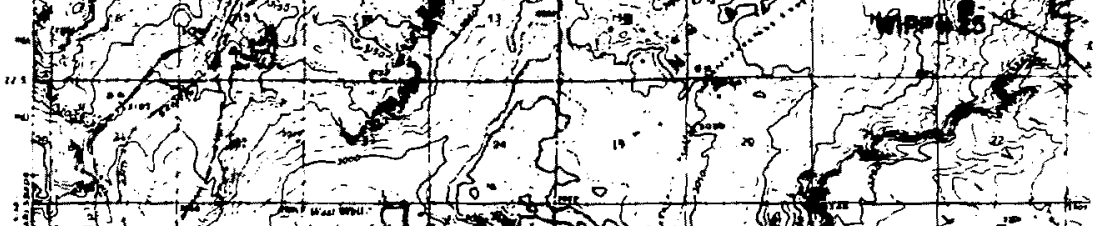
(f)

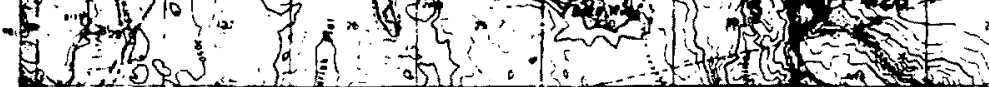

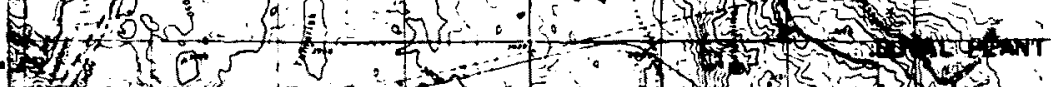

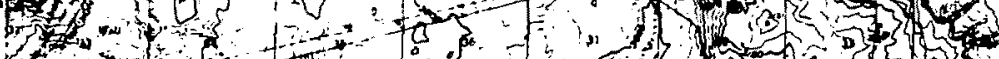
4. 


\section{Drilling Parameters and Expected Drilling Requirements}

The following drilling program is intended to be typical for each of the six Nash Draw boreholes, WIPP 25 through 30 . The diameters of the holes and drilling techniques will be basically similar, but the depths of formations and total depths will naturaliy vary according to location. For guidance on depth projection, refer to previous section containing genlogic prognosis.

1. Level and prepare a suitable surface location compatible with local terrain and drilling equipment selected.

2. Locate torehole according to surveyed location and establish concrete monument with brass cap in the imediate vicinity of borehole such that it can be utilized as the datum point for all vertical measurements of the drilling, coring and logging activities. Once established, this marker should be surveyed by a certified land surveyor to establish vertical elevaiion of the benchmark within \pm one foot of nearest National Geodetic Survey and the lateral coordinates within \pm one foot with respect to nearest section corner.

3. Move in rig and rig up in a manner such that a continuous core can be taken from as near the surface as practicable to the total depth. The core should be at least 3-5/8" diameter or the largest practically obtainable in continuous coring operation. Utilize a drilling and circulating fluid best suited for promoting $f u l l$ core recovery. Lse additives as required to control loss of circulation keeping in mind the desire to convert these boreholes into hydrologic observation wells. All core is to be logged, marked, and handled according to procedures outlined in section III.A.

4. Dury geologist will closely monttor core production and establish total depth. Total depth is expected to be about 50 Eeet below the top of salt and past the evaporite residuum at the Top of the Salado Formation.

3. L'pon reaching total depth, borehole will be conditioned and made ready for geophysical logging by a commercial firm and USGS/WRD if they desire. Borehole may have to be opened to accomodate logging cools.

6. Cieoph $: s i c a l$ logs are to be run to provide informution for:

a. Identifying rock types encuuntered

b. Aiding the maintenance of stratigraphic control

c. Aid in verifying core depths

d. Identifying fluid bearing zones 


\section{e. Evaluate relative permeabilities}

f. Up-hole sonic velocity

g. Letermine elastic properties of rocks.

The actuai commercial logs selected to produce the above information will be established at a later time after a better understanding of the hole conditions is known. Procedures for logging proyram will be found in Section IV.B.

7. After logging operations are completed, the borehole should be made ready for running casing and cementing. A string of 5-1/2", 15.5, J-55 casing (or reasonable equivalent) with centralizers will be scood and cemented to the surface. A prime reason for casing is to isolate aquifers from one another and proceduras described is: Section III must be followed.

8. Upon completion of Lasing and cenenting, the well should be filied with fresh water and covered with a temporary, removable cap.

9. Clean up pad areas, fill in pits and leave hole temporarily abandoned for hydrological testing.

D. Discussion of potential hazards

$\therefore$ unusual hazards are expected. Some small inert ges p-ikets ras. h. encountered though unlikely above the salt. 
III. FIELD OPERATING PROCEDURES FOR QUALITY CONTROL REQUIREMENTS

Portions of this field activity are consldered of such a signiflcance that quality control measures have been established and these are subject to independent audit by Sandia Laboratory Qualf.ty Assurance personnel as well as NRC Field Audit Team. These activities are:
A. Measurement of Surface Location and Elevation
B. Core Logging and Handling and Storage
C. Geophysical Logging
D. Casing and Cementing.

The atove activities will be monitored in detail or reviewed by designated quality control experts whose education, knowledge and/or experience makes him qualified to assure adherence to operating procedures or to ascertain or certify that desired objectives have been met.

when designaced, this individual shall take appropriate measures to negotiate with pertinent officials to whatever extent necessary to assure acceptable results. 
July 26,1978

A. Measurement of Surface Location and Elevation

The general location wll be established by Division 531 following a review of resistivity surveys, surface features, accessibility and other geo-political considerations. A preliminary land survey shal be conducted by a Registered Land Surveyor to establish access routes and set srakes for drill location and pad boundaries. Dimension of che location will be established with nearest section boundaries and nearest marked section corners to provide data necessary for obtaining land use permits. Drawings or sketches suitable for construction use shall be subuitced. After pad construction is complete and at the time of, hole spudding, a concrete monument with a brass cap will be set in the immediate vicinity of the borehole such that it can be used as the datum point for all borehole vertical measurements, Once this conument is established, this monument and itg lateral relation to borehole axis is to be surveyed by a Registered land Surveyor to establish the vertical elevation within \pm 1 ' of the nearest NGS monument and the lateral coordinates within $+l^{\prime}$ with respect to nearest section boundaries and recoverable section corners.

Copies of all field notes utilized in conducting the "as-built" survey, as well as a written description of techniques and instruments utilized in making the survey shall be submicted along with survey diraings carryings the stamp of the responsible surveyor. 
B. Core Logging and Handling and Scorage

A duty geologist will $\log$ and measure core as it is removed from core barrei. When drill cuttings are required, duty geologist will see they they have been taken, washed and dried. They should then be tied in $100^{\circ}$ bundles, boxed, and marked with well identity, and interval taken. Cuttings will then be taken to core storage in Carlsbad along with core.

A record should be kept showing date and tour, sequence of core interval, depth of interval, footage of core rezovered, and percentage. If significant intervals are missing, the depth and interval of missing cure should be recorded as well as any determinable physical properties of the formation. Rig operating conditions such as RPM, weight on bit, circulating pressure should also be kept.

For sake of consistency, a routine has been established for handing and marking core at the drill pad as follows:

1. Coring contractor and roustabouts will lay barrel down and open barrel. The dury geologist will supervise removal from core barrel and placement in troughs in the order they come out of barrel for inspection and measurement. Troughs are marked orange or red indicating top end and black indlcating down direction.

2. If core is suicable for marking, each major piece should be marked with a waterproof, black-ink arrow pointing in the direction the hole is advancing. If core is not suitable for marking, the above is to be marked on sleeving using an indelible, waterproof, black marking pen.

3. Log, identify and measure all core pieces, mark to the nearest foot relative to measurements of drill pipe, express to closest 1/10th of foot. Note: all depth measurements are from the top side of the Kelly Bushing unless otherwise specified.

4. Move troughs to core shed and separate into appropriate lengths. Sleeve and seal and insert into boxes. Tape boxes and mark outside of box with hole identity and depths of core interval.

5. Transport the boxed core to core storage, taking particular care in handing and delivery to avoid core damage.

6. The duty geologist will de responsible for assuring corruct markings un the exterior of all core boxes, safe packaging, safe transport from drill pad location to core storage location, and making entries into the Daily Core Logging Record (sample follows) as to quantities and date of core de:ivery to core storage. 


\section{DAILY CORE LOGGING RECORD}

Dáce

DUTY GEOLOGIST

LOG HEADINGS :

Company

kell Number

WIPP No.

Fit $1 \mathrm{~d}$ County

State New Mexico

location

Section

Township

Range

Pertwinen: Datun. Elevations: K.B.

Lril]ing Néasured From

D.F.

G.L.

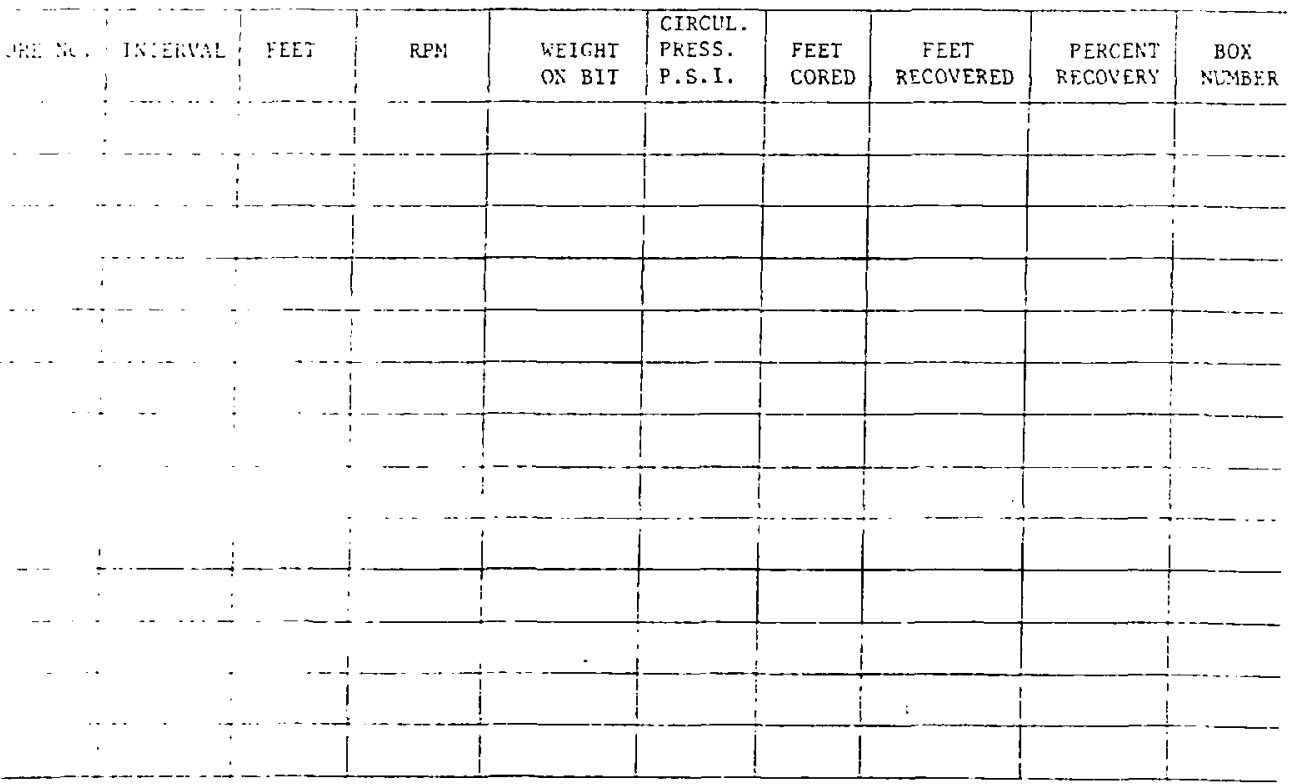


July 26,1978

C. Geophysica1 Logging

1. Prior to $108 g 1 \pi g$, a Sandia representative will weet with the logging engIneer, present "Iastruct lons to Logging $\mathrm{CO}^{\text {" as show }}$ on following pages, and discuss:

a. The entire logging program and any special requirements

b. Hole conditions that may cause problems

c. Zones of special interest.

2. The equipment $\mathbf{w 1 1}$ be "varwed up" for the adequate anount of time and tools will be checked to see that they are functioning properly upon arrival at the location.

3. Rm, Rmf, and Rmic will be measured on mud samples. Estimated values are not acceptable. The service company ohould run the sample through a mud press.

4. Proper scales w1ll be used on each log.

5. AlI Sidewall and Compensated Neutron logs and all density porosity curves will be run on limestone matrix over the zones of interest. regardiess of the 11thology.

6. Equipgent will be cested while runging In hole.

7. Before and after $\log$ calibrations will be shown for all curves.

8. Panel calibrations will be shown for all density and neutron logs, integration checks will be shown for all integrated Acoustic logs.

9. In addicion to caliper rings the caliper callbration should show "tool full open" and casing readings.

10. A mininum 200 feet repeat must be shown.

11. Overlap previous runs by at least $200 \mathrm{feet}$.

12. All headings information will be completely filled out.

13. A "Log Quality Report" such as is shown on following pages will be completed by qualified Sandla representarive. 
Field Print

CHECK ALL BOXES - ACCEPTABLE YES OR UNACCEPTABLE NO

Sectione not opplicable to a particuter survice. Leave Blank.

4. HEADINC

I. Corres Heading Usod

2. Heding Cuta Properly Completed

3. Equipment Uned Section Comploted

4. Equipment Dato Soction Completed

5. Scale Changes Nored an Hoading

6. Are all obnarmal canditione enplained in the emerts vection

b. calibrations and scales

i. Seales Correce for Arrea

2. Scalen Labellod

]. Scale Chonges Lobelled

4. Zorops Recoroud

5. B.toro Lae Cattibetiant

b. Alter Log Calibratiene

1. Repeot Section Recozded

B. Ropeof Saction Accopleble

c. VALIDITY OF LOC

1. Curver Functioning Cotastis

2. Do Leg velues fall mithin reoseneble limits

3. Curver on Oessh

2. Logging Speed Indiceted

5. Logging Speed Corract

c. appearance

1. Pringing of Troune Noet

2. Primting of Tyoung aceveste

3. Crid and Pon Traces

4. Solices Sireight and Clean

5. Film Coucesty Procenent

ه. Cenotel Print Oudity

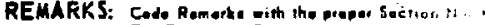

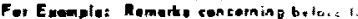
cellthretlons wauld be coded B.5.
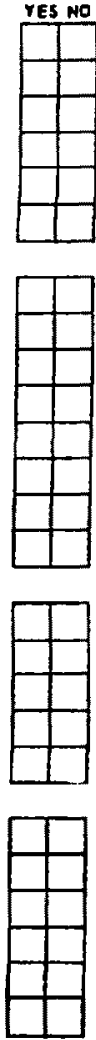
FENIX SCISSON, INC.

\section{INSTRUCTIONS TO LOGGIVG COMPANY}

Date

$$
\begin{aligned}
& \text { Losging Company } \\
& \text { Logging Englneer } \\
& \text { Witnessed By }
\end{aligned}
$$

\section{Log Headings:}

Company Fenix seisson, Inc. for Sandia Laboratories

Well Number WIPP No.

Field

County State Nex Mexico

Locktion

Section

Township

Range

Permarent Datuc Elevetions: K,B

Log Measured From

D, F .

Drilling Heasured Frod

G.L.

\section{$-$ \\ Casings \\ Type Juid in Borehoie}

Hole Status: SIZE FROM TO

SIZE FHOM TO

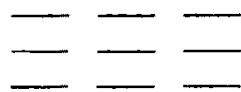

Borehole

Densicy Fluid Level

Viscosity $\mathrm{pH}$

Fluid Loss

Purpose of Hogging Program, Zones of Special Interest, Critical Hole Conditions, Remarks, Etc.

Number of Prints: Field

Final

Invoice To: Fenix \& Scisson, Ine.

401 \%. Canel Street

Carlebad, $\mathrm{NM} 8 \mathrm{~B} 220$ 
Fenix \& Scisson, Inc.

Instructions to Loging Company

LOg. iNo.

(a) Vertical Depth Scales 2-inches/100-feet and 5-inches/100-feet

(b) Herizontal Logging Scgies

(c) LogBing Speed Desired

(a) Interval to be Loged

(e) Zones of Special Interest

(r) Speciel Instruction

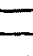


D. Casing and Cementing

1. Inspect casing to be run. Sand blast if necensery to rewove eevere rust flakes. Pite of rust without flakes way actualiy enhance bonding.

2. Condition hole, ream 1 necessary to remove tight places.

3. Condition fluid until mostly free of cuttings.

4. Ins-all a combination float shoe.

5. Stand casing with centralizer. Utilize loga to determine location as well as routine apacing of 60-90 feet apart.

6. Begin pumplog and displace well fluid with wud flush, then get, and cement sluriy. Voe $70-30 \mathrm{poz} \mathrm{mlx}$, salt to saturation, and $2 \%$ bentonite gel.

7. Displacement rate approximately $2-1 / 2$ barrelo per minute.

8. Bump plug with approximately 500 p51 over pumping-mixing rate. M1x 1000 psi, close in head.

9. WOC 48 hours.

10. N1pple up after 36 hours.

NOTE: Observe regulations 1ssued by State Englneer; In particular, requirement that "casing shall not be lnstalled or cemented without prior notification to the State Engineer office." 
July 26,1978

IV. REPORTS

Distribution Instructions:

A. Daily Reports

F\&S, Carlsbad, shall provide to Sandia, Carlsbad, a copy of the daily report. Sandia, Carlsbad, will telefax weekdays to D. Schueler, DOE/ALO, and L. R. Hill, 5311, Albuq., who will mail copies to McFarling, 9517, Seward, 1135, and Statler, 1133. A copy of the daily report is to be kept by Sandia, Carlsbad.

B. Hole History

Compiled by F\&S, Carlsbad, and Las Vegas, NV. Send copy to

R. D. Statler, 1133, for Sandia, Albuquerque, distribution.

C. Geophysical Logs

F\&S, Carlsbad, shall obtain nine copies of field prints and make general distribution, to include the following:

1 - Sandia, Carlsbad

I - 5 . Jones, USGS, Spec. Proj. Div., Denver, Co

1 - J. Mercer, USGS/WRD, Albuq., NM

1 - G. O. Eachman, USGS/WRD, Albuq., NM

1 - D. W. Powers - 5311, SLA

4 - F\&S, Carlsbad

FsS, Carlsbad, shall order 19 Final copies for distribution as follows:

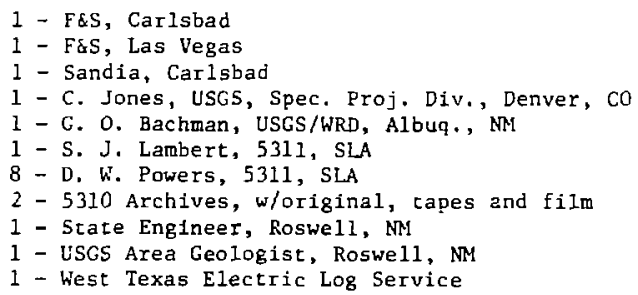

D. Miscellaneous Records shall be provided by $r \& S$, Carlsbad, including the following, with original to le kept on file at F\&S, Carlsbad, and one copy to Sandia, Carlsbad:

Drillers Logs, Bit Records, Drill Fluid Recaps Equipment Certification Drilling History Chart Cost Records (copj also to R. D. Statler, 1133, Albuq.) 


\section{Sandia Laboratories}

date August 18, 1978

Albuquerque. New Mexico

10 Distribution

Lwermore. Caltornsa

from: R. D. Seatler: 1133

Sutert Supplement 1 to Fleld Operating Program WIPP 25 thru 130

The following additions and modifications to the Fleld operating Program for the Nash Draw Exploratory Wells, WIPF $/ 25$ thru 30 are forwarded for your use and information:

A. Fourteen (14) copies of the As Bullt Survey of the hole location should be distributed as follows:

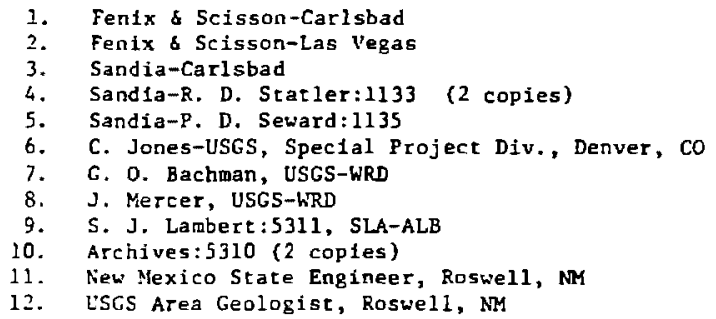

B. 1. Run Geophysical logs up to the highest level that will hold fluid. Estimated to be \pm 220 -feet.

Logs to be run as follows:

3. Densilog

$?$ BHC Acoustilog

3. Micro Laterolog

4. Dual Läterolog

5. Compensated keutron

6. "Up Hole Velocity"

2. Add J. Mercer, LSCS-kRD to distriburion of all logs.

$\therefore$ Core Logging, Handling and Starage procedures are modjified to include photography of core a the drill site as soon to it has been logged and Identifled by the Duty Geologist and before it is sleeved for storage.

: : 5 of all core photographs are to be made by Sandia Laboratories $\because \cdots:-$ isd as follons:

: ropics - Division S311

- Copy - C. L. Jones, USGS, Denver, CO

I Copy - G. O. Bachman, USGS-WRD 
APPENDI $X \mathrm{C}$

HOLE HISTORY

by

R. D. Statler

Division 1133

and

P. D. Seward

Division 1135

Sandi a Laboratories 
INTRODUCTION TO APPENDEX C, HOLE HISTORY

The hole histary is a document provided soon after completion of the borehole, and it sumarizes the relevant information on the daily log kept by the contractor. The hole history is not edited to ensure conformance in every detail with later information developed for previous chapters. Further information may be obtained as necessary through examination of the original daily time logs. 


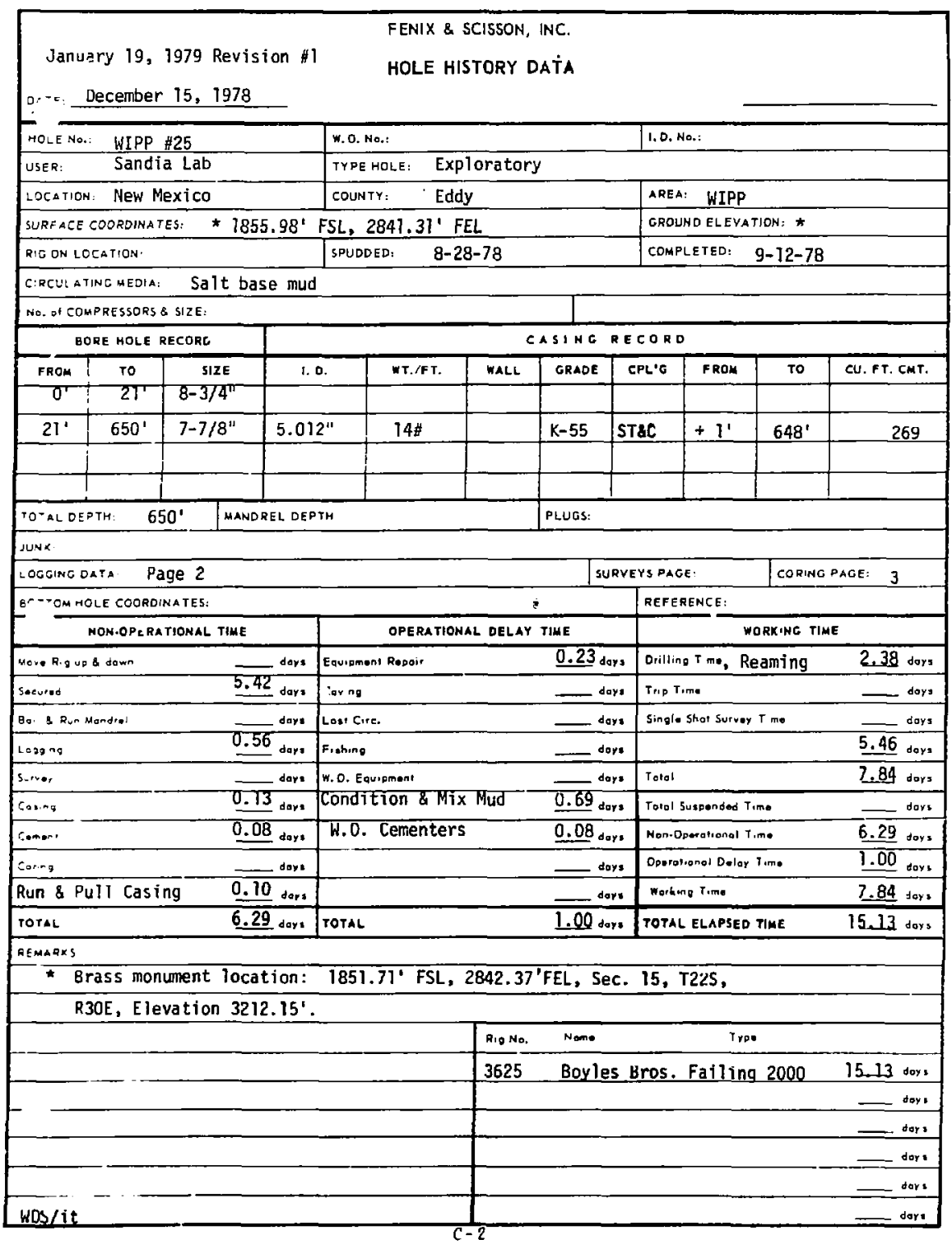




\section{WiFP : $: 25$ \\ HOLE HISTORY}

$8-28-78$

$8-29-78$

$?-3 n-7 \&$

$9-31-75$

$9-1-7 \varepsilon$

$s-2-78$

$2-5-78$

$9-6-7 \delta$

$9-7-7 \varepsilon$

$9-8-73$

$9-9-78$

$?-11-7 ?$

$9-12-75$
Hoved in Boyles Brothers rig $\$ 3625$ and rigged up. Cut core ${ }^{\prime \prime} 1$ and $\# 2$ from $0^{\prime}$ to $3^{\prime}$ using a $3^{\prime \prime} \times 1.5^{\prime}$ split spoon. Cut core $\# 3$ thru $\#^{\prime}$ from $3^{\prime}$ to $21^{\prime}$ using a $3^{\prime \prime} \times 3^{\prime}$ steel Pitcher tube.

Opened $3^{\prime \prime}$ hole to $8-3 / 4^{\prime \prime}$ from $0^{\prime}$ to $21^{\prime}$ using air. Set 7" $0 . D$. casing at $21^{\prime}$ and packed off at surface. Mixed up salt base mud. Made up $3.937^{\prime \prime} \times 2.4^{\prime \prime}$ core bit and cut core \#9 thru core ${ }^{\prime \prime} 17$ from $21^{\prime}$ to $88^{\prime}$ and lost circulation.

Mixed mud and lost circulation materials. Cut core $\# 18$ thru I:27 from $38^{\prime}$ to $168^{\prime}$.

Cut core $\$ 28$ thru \#34 from $168^{\prime}$ to $238^{\prime}$.

Opened 3.937" hole to 5-7/8" from $21^{\prime \prime}$ to $238^{\prime}$. Set 4-1/2" 0.0 . , Hhl casing at $238^{\prime}$. Cut $3.937^{\prime \prime}$ core $\# 35$ thru $\# 45$ from $238^{\prime}$ to $348^{\prime}$.

Cut core $\frac{7}{7} 46$ thru $\frac{1}{7} 48$ from $348^{\prime}$ to $374^{\prime}$. Secured rig at 0600 hours.

Rig secured from $9-2-78$ to 0600 hours on 9-5-78. Cut core $\$ 49$ thru $\frac{156}{15}$ from $374^{\prime}$ to $451^{\prime}$.

Cut core $\# 57$ thru $\# 78$ from $451^{\prime}$ to $603^{\prime}$.

Cut core $\# 79$ thru $\# 84$ from $603^{\prime}$ to $650^{\prime}$. Ran USGS logs. Pulled $4-1 / 2^{\prime \prime} 0.0$., HW casing out of the hole. Opened $5-7 / 8^{\prime \prime}$ hole to $6-1 / 8^{\prime \prime}$ from $21^{\prime}$ to $238^{\prime}$ and $3.937^{\prime \prime}$ hole from $238^{\prime}$ to $395^{\prime}$.

Opened 3.937" hole to 6-1/8" from 395' to $650^{\prime}$. Opened 6-1/8" hole to $7-7 / 8^{\prime \prime}$ from $21^{\prime}$ to $195^{\prime}$.

Opened 6-1/8" hole to 7-7/8" from $195^{\prime}$ to $395^{\prime}$. Secured rig at 0600 hours.

Pig secured from $9-9-78$ to 0800 hours on $9-11-78$. Opened 6-1/8" hole to 7-7/?" from $395^{\prime}$ to $650^{\circ}$. Laid down drijl pipe. Ran Dresser ftlas logs.

Completed logging. Ran 5-1/2" 0.0., K-55, 14\# casing and set at $647.71^{\circ}$. Cenented arrulus to surface using Dowe11 with 24 barrels of mud wash and 5 barrels of water ahead of 50 sacks (77 $\mathrm{ft}^{3}$ ) of RFC cement and 150 sacks (192 $\mathrm{ft}^{3}$ ) of $70 \%$ class " $\mathrm{C}$ " cement, $30 \%$ Litepoz $3,2 \%$ gel, $2 \%$ calcium chloride, $0.1 \%$ salt dispersant and $19,4 \mathrm{H}$ per sack of salt. Cement in place at 1638 hours. Hole completed. 
Page 2

II PP $: 25$

HOLE HISTORY

LOG INDEX SHEET

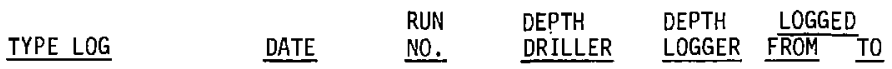

DRESSER ATLAS LOGS

BHC Acoustilog

Compensated Densilog

Dual] Laterolog

llicro-Laterolog

Compensated Neutron

Gamma

$\begin{array}{llllll}9-12-78 & 1 & 655 & 653 & 0 & 647 \\ 9-12-78 & 1 & 655 & 652 & 0 & 651 \\ 9-12-78 & 1 & 655 & 653 & 0 & 652 \\ 9-12-78 & 1 & 655 & 653 & 0 & 650 \\ 9-12-78 & 1 & 655 & 655 & 0 & 654\end{array}$

Seismic Survey

SEISMIC REFERENCE SERINE LOG

9-12-78 $1 \quad 655$

653

0

647

NOTE: Logs furnished F\&S/Mercury.

C -4 


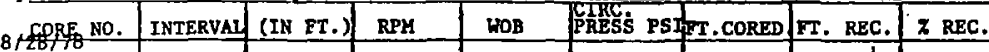

\begin{tabular}{|c|c|c|c|c|c|c|c|c|c|}
\hline 1 & 0 & 1.5 & USTNG 1 & 5' SPLIT & SPOON & 1.5 & $1.5^{t}$ & $100 \%$ & \\
\hline 2 & 1.5 & 3.0 & 11 & I $\quad \therefore$ & " & 1.5 & $1.5^{\frac{1}{t}}$ & $100 \%$ & \\
\hline 3 & 3,0 & 6.0 & USING & - & & 3.0 & 3.0 & $100 \%$ & \\
\hline 4 & 6.0 & 9.0 & $\because n$ & 4 & 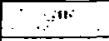 & 30 & 3.0 & 1002 & \\
\hline 5 & 9.0 & 12.0 & $\because *$ & $\because \cdot$ & $n$ & $3: 0$ & $3: 0$ & $100 z$ & \\
\hline 6 & 12.0 & 15.0 & $1 \quad \therefore$ & 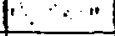 & $"$ & 3.0 & 3.0 & 1007 & \\
\hline 7 & 15.0 & 18.0 & & 11 & $"$ & 3.0 & 3.0 & $200 \%$ & \\
\hline 8 & 18.0 & 21.0 & $"$ & 11 & $"$ & 3.0 & 1.7 & & \\
\hline 129) 9 & 21 & 26 & 250 & 2000 & 100 & 5 & 5 & $100 \%$ & 1 \\
\hline $10^{-6}$ & 26 & 32 & 200 & 2000 & 150 & 6 & 4.7 & $78 \%$ & 1,2 \\
\hline 11 & 32 & 36.8 & 200 & 2000 & 150 & 4.8 & 4.5 & 947 & 2 \\
\hline 12 & 36.8 & 41 & 200 & 2000 & 150 & 4.2 & 4.5 & $107 \%$ & 3 \\
\hline 13 & 41 & 50 & 200 & 3000 & 200 & 9 & 9 & $100 \%$ & 3 \\
\hline 14 & 50 & 60 & 200 & 3000 & 200 & 10 & 9.7 & $97 \%$ & 4 \\
\hline 15 & 60 & 70 & 200 & 3000 & . 200 & 10 & 10 & $100 \%$ & 5,6 \\
\hline 16 & 70 & 78 & 200 & 3000 & 200 & 8 & 8 & $100 \%$ & 6 \\
\hline 17 & 78 & 88 & 200 & 3000 & 200 & 10 & 9.9 & $99 \%$ & 7 \\
\hline $8 / 3018$ & 88 & 90 & 200 & 4000 & 200 & 2 & 1.7 & $85 \%$ & 8 \\
\hline 19 & 90 & 98 & 200 & 4000 & 200 & 8 & 7.6 & $95 \%$ & 8 \\
\hline 2 & 98 & 108 & 200 & 4000 & 200 & 10 & 10 & $100 \%$ & 91 \\
\hline 21 & 108 & 118 & 200 & 4000 & 200 & 10 & 10 & $100 \%$ & 10 \\
\hline 22 & 118 & 128 & 200 & 2500 & 300 & 10 & 9.75 & $97.5 \%$ & 11,12 \\
\hline 23 & 128 & 130.5 & 200 & 2500 & 350 & 2.5 & 2.9 & $116 \%$ & 12 \\
\hline 24 & 130.5 & 140 & 200 & 3000 & 300 & 9.5 & 9.1 & $96 \%$ & 12,13 \\
\hline 25 & 140 & 150 & 200 & 3000 & 300 & 10 & 10 & $100 \%$ & 13,14 \\
\hline 26 & 150 & 160 & 200 & 3000 & 300 & 10 & 10 & $100 \%$ & 14,15 \\
\hline 27 & 160 & 168 & 200 & 3000 & 200 & $\bar{B}$ & 8.2 & $103 \%$ & 16 \\
\hline $8 / 31 / 28$ & 168 & 178 & 200 & 3000 & 200 & 10 & 9.8 & $98 \%$ & 17 \\
\hline 29 & 178 & 188 & 200 & 3000 & 200 & 10 & 10.1 & $101 \%$ & $18,19,2$ \\
\hline 30 & 188 & 198 & 200 & 3000 & 200 & 10 & 9.7 & $97 \%$ & 20 \\
\hline 31 & $1980^{\circ}$ & 208 & 200 & 3000 & 200 & 10 & 10.2 & $102 \%$ & 20,21 \\
\hline 32 & 208 & 218 & 200 & 3000 & 200 & 10 & 9.9 & $99 \%$ & 21,22 \\
\hline 33 & 218 & 228 & 200 & 4000 & 200 & 10 & 10.1 & $101 \%$ & 22,23 \\
\hline 34 & 228 & 238 & 200 & 4000 & $175-200$ & 10 & 10 & 1007 & $23,24,2$ \\
\hline$9 / 1 \longdiv { 3 5 }$ & 238 & 248 & 200 & 4000 & 200 & 10 & 8.7 & $87 \%$ & 25,26 \\
\hline 36 & 248 & 258 & 200 & 4000 & 300 & 10 & 10 & $10 \overline{0 \%}$ & 26 \\
\hline. & 258 & 268 & 200 & 4500 & 300 & 10 & 20 & $100 \%$ & 27,28 \\
\hline 38 & 268 & 278 & 200 & 4500 & 300 & 10 & 10 & $100 \%$ & \\
\hline 39 & 278 & 288 & 200 & 4500 & 300 & 10 & 10 & $100 \%$ & 29,30 \\
\hline 40 & 288 & 298 & 200 & 4500 & 300 & 10 & 10 & $100 \%$ & 30,31 \\
\hline
\end{tabular}


NIPE 25

- PAGE 2

\begin{tabular}{|c|c|c|c|c|c|c|c|c|c|}
\hline CORE NO. & INTERVAI & (IN FT.) & RPH & HOB & PRESS' PS I & FT. CORED & FT. REC. & I REC. & \\
\hline 41 & 298 & 308 & 200 & 4500 & 300 & 10 & 10 & $100 \%$ & 31,32 \\
\hline 42 & 308 & 318 & 200 & 4500 & 300 & 10 & 10 & $100 \%$ & 32,33 \\
\hline 43 & 318 & 328 & 200 & 4500 & 300 & 10 & 10.2 & $1027, \alpha$ & $\therefore 33,34$ \\
\hline 44 & 328 & 338 & $\therefore 200$ & $: 4500^{\circ}$ & 300.7 & $10 \therefore$ & $\therefore 9 ; 8 \cdots$ & $\therefore 982.7$ & $134,55^{\circ}$ \\
\hline $9 / 2 / 78$ & & & $\therefore \quad \therefore$ & $\because \cdots$ & $\because$ & $\therefore$ & 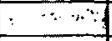 & $5+24$ & $3 \cdot 87$ \\
\hline 45 & 338 & 348 & $200^{\circ}$ & 4500 & 300 & 10. & 10.2 & $1027 \div$ & 2335,36 \\
\hline 46 & 348 & 358 & 200 & 4500 & 300 & 10 & 10.1 & $101 \%$ & 37,38 \\
\hline 47 & 358 & 368 & 200 & 4500 & 300 & 10 & 9.9 & $99 \%$ & 38,39 \\
\hline 48 & 368 & 374 & 200 & 4500 & 300 & 6 & 6 & $100 \%$ & 39 \\
\hline \multicolumn{10}{|l|}{$9 / 5 / 78$} \\
\hline 49 & 374 & 384 & 200 & 4500 & 300 & 10 & 10 & $100 \%$ & 40 \\
\hline 50 & 384 & 393 & 200 & 4500 & 300 & 9 & 9 & $100 \%$ & 41 \\
\hline 51 & 393 & 403 & 200 & 4500 & 300 & 10 & 10 & $100 \%$ & 42,43 \\
\hline 52 & 403 & 413 & 200 & 4500 & 300 & 10 & 10 & 1007 & 43,44 \\
\hline 53 & 413 & 423 & 200 & 4500 & . $\quad 300$ & 10 & 10 & $100 \%$ & 44,45 \\
\hline 54 & 423 & 433 & 200 & 4500 & $30 n$ & 10 & 10 & $100 \%$ & 45,46 \\
\hline 55 & 433 & 443 & 200 & 4500 & 300 & 10 & 10.1 & 101 & 46,47 \\
\hline \multicolumn{10}{|l|}{$9 / 6 / 78$} \\
\hline 56 & 443 & 451 & 200 & 2000 & 300 & 8 & 7.5 & $94.7 \%$ & 48 \\
\hline 7 & 451 & 457.5 & $"$ & $"$ & $"$ & 6.5 & 6.2 & $95 \%$ & $49:$ \\
\hline 38 & 457.5 & 458 & " & $"$ & $"$ & .5 & .4 & $80 \%$ & 49 \\
\hline 59 & 458 & 464 & " & $"$ & $"$ & 6 & 6 & $100 z$ & 50 \\
\hline 60 & 46: & 466.5 & "t & $: *$ & $n$ & 2.5 & .8 & 327 & 50 \\
\hline 61 & 466.5 & 468.5 & $"$ & $"$ & " & 2.0 & 1.4 & $70 \%$ & 51 \\
\hline 62 & 468.5 & 470 & 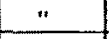 & $*$ & $"$ & 1.5 & 1.0 & 66.67 & 51 \\
\hline 63 & 470 & 472.5 & 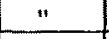 & $"$ & " & 2.5 & 2.5 & $100 \%$ & 51 \\
\hline 64 & 472 & 482 & ". & $"$ & $"$ & 10 & 10 & $100 \%$ & 51,52 \\
\hline 65 & 482 & 492 & $"$ & $"$ & $" 8$ & 10 & 10 & $100 \%$ & 52,53 \\
\hline 66 & 492 & $502 .^{\circ}$ & " & $"$ & " & 10 & 10 & $100 \%$ & 54,55 \\
\hline 67 & 502 & 512 & $"$ & " & 1 & 10 & 10 & $100 \%$ & 55.56 \\
\hline 68 & 512 & 522 & $"$ & $" 1$ & $n$ & 10 & 8.6 & 867 & 56,57 \\
\hline 69 & 522 & 526 & 200 & 2000 & 200 & 4 & 3.8 & $70 \%$ & 57 \\
\hline 70 & 526 & 533 & $"$ & $"$ & * & 7 & 6.1 & $87 \%$ & 57,58 \\
\hline 71 & 533 & 537.5 & " & $"$ & $"$ & 4.5 & 3.8 & 847 & 58 \\
\hline 72 & 537.5 & 544 & $"$ & $"$ & $"$ & 6.5 & 5.5 & $84.6 \%$ & 58,59 \\
\hline 13 & 544 & 553.5 & $"$ & $"$ & " & 10 & 10 & $100 \%$ & 59.60 \\
\hline ; & 553.5 & 563.5 & $"$ & $"$ & " & 10 & 10 & $100 \%$ & 60,61 \\
\hline 75 & 563.5 & 573 & $"$ & 1 & 1 & c & 9.5 & 1007 & 61,62 \\
\hline 76 & 573 & 583 & $"$ & " & 1 &.$U$ & 10 & 1007. & 63,64 \\
\hline 77 & 583 & 593 & 200 & 2000 & 300 & 10.2 & 10.2 & $100 \%$ & 64,65 \\
\hline
\end{tabular}


-PAGE 3

\begin{tabular}{|c|c|c|c|c|c|c|c|c|c|}
\hline CORE NO. & INTERVAY & (IN FT.) & RPY & hOB & PRRCS PS & FT. CORED & ET. REC. & 7 REC. & \\
\hline \multicolumn{10}{|l|}{$9 / 7 / 78$} \\
\hline 78 & 593 & 603 & 200 & 2000 & 300 & 9.8 & 9.3 & 94.87 & 65,66 , \\
\hline 79 & 603 & 613 & 200 & 3000 & 300 & 10 & 10.2 & $1027:$ & $-66,67$ \\
\hline 80 & 613.2 & 615 & $\because 200 \therefore \therefore$ & 3000 & $300 \cdots$ & $\because 1,8 \%$ & $\because 1,80$ & 1002 & $\therefore 679$ \\
\hline 81 & 615 & $\because 625$ & 200 & 3000 & $300 \%$ & $10:-\cdots$ & $10.1 *$ & $101 \%$ & $=67,68$ \\
\hline 82 & 625 & 635 & $200^{\circ}$ & 3000 & 300 & 10 & 9.9 & $99 \%$ & 69,70 \\
\hline 83 & 635 & 645 & 200 & 3000 & 300 & 10 & 10 & $100 \%$ & 70,71 \\
\hline 84 & 645 & 650 & 200 & 3000 & 300 & 5 & 5.3 & $106 \%$ & 71 \\
\hline & & & & & & & & & \\
\hline \multicolumn{10}{|l|}{$\approx$} \\
\hline & & & & & & & & & \\
\hline & & & & & & & & & \\
\hline & & & & & & & & & \\
\hline & & & & & & & & & \\
\hline & & & & & . & & & & \\
\hline & & & & & & & & & \\
\hline & & & & & & & & & \\
\hline & & & & & & & 는 & & \\
\hline & & & & & & & & & \\
\hline & & & & & & & & & $:$ \\
\hline & & & & & & & & & \\
\hline & & & & & & & & & \\
\hline & & & & & & & & & \\
\hline & & & & & & & & $\cdot$ & \\
\hline & & & & & & & & $\cdot$ & \\
\hline & & & & & & & & & \\
\hline & & & & & & & & & \\
\hline & & & & & & & & & \\
\hline & & $\therefore$ & & & & & & & \\
\hline & & & & . & & & & & \\
\hline & $\because$ & & & & & & & & \\
\hline & & & & & & & & & \\
\hline & & & & & & & & & \\
\hline & & & & & & & & & \\
\hline & & & & & & & & & \\
\hline & & & & & & & & & \\
\hline & & & & & & & & & \\
\hline & & & & & & & & & \\
\hline & & & & & & & & & \\
\hline & & & & & & & & & \\
\hline & & & - & & $\bar{C}-$ & & & & \\
\hline
\end{tabular}




\section{SURVET MONUMENT "AS MUMT" YURP25 \\ SECTION LF, TOWNSHP2RS RANGE 30E MMPM EDOr COUNTY, MTW MIXXKCO}

ELEVATION OF BRASS MOMUMENT 3212.15' $1851.71^{\circ}$ FSL Q 2842,37FEL

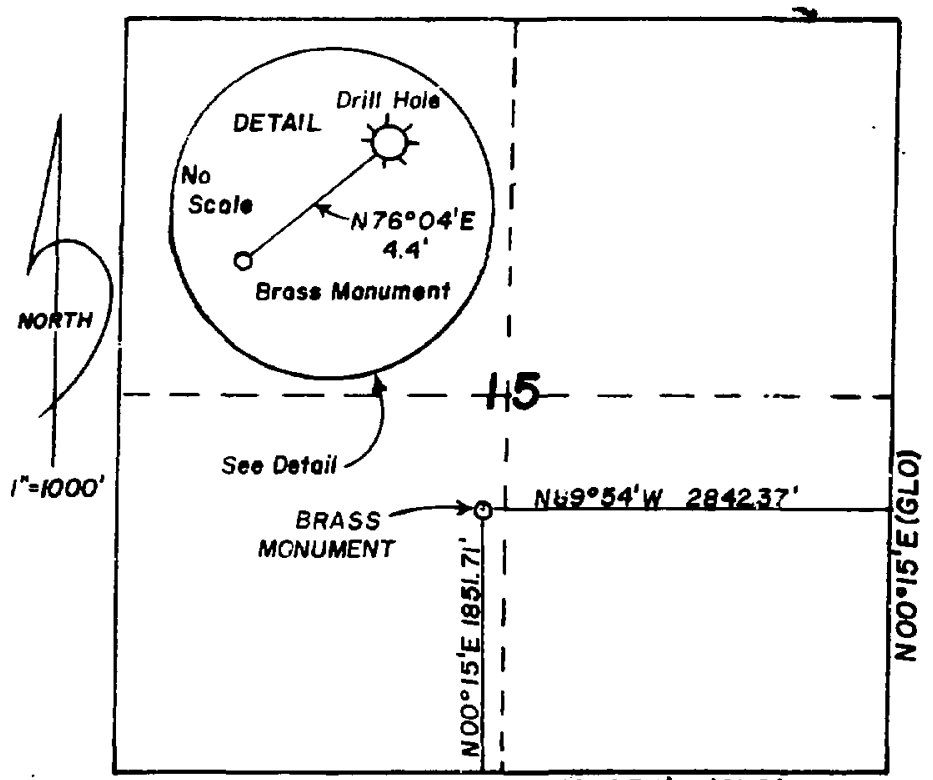

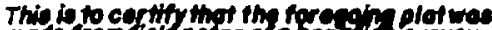
$N 89^{\circ} 54^{\prime} W(G L O)$

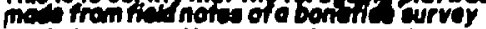
mece by me andis true and correct to the

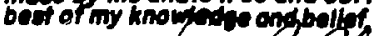
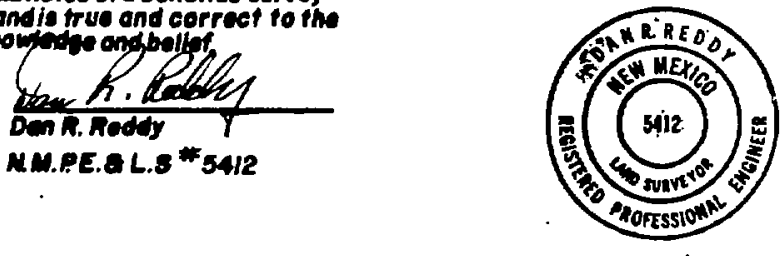


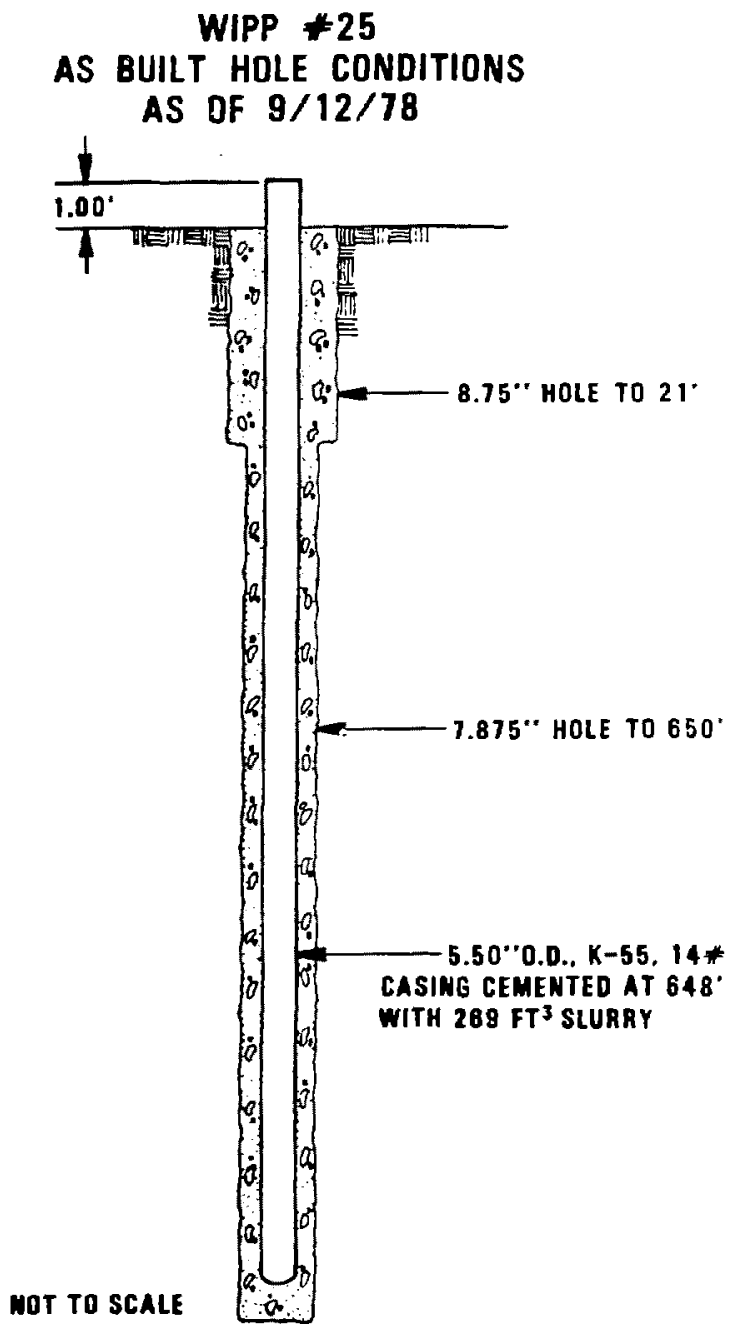

C-9 
APशENDIX D

LOGS

by

S-E. Shafer

Division $45 L 1$

Sandia Laboratories 
WI PP 25 Logs 1

\begin{tabular}{|c|c|c|c|c|c|}
\hline $\log$ & Company & ELS I* ${ }^{2}$ & $\begin{array}{l}\text { Top of } \\
\text { Logged } \\
\text { Inter val }{ }^{3} \\
\text { (feet) }\end{array}$ & $\begin{array}{c}\text { Eottom } \\
\text { Logged } \\
\text { Inter val } \\
\text { (feet })\end{array}$ & Date \\
\hline BHC ACOustilog & Dresser Atlas & W7480x & Surf. & 647 & $9 / 12 / 70$ \\
\hline $\begin{array}{l}\text { Compensated } \\
\text { Denst } 1 \text { og }\end{array}$ & Dresser Atlas & W7480S & SurE. & 651 & $9 / 12 / 78$ \\
\hline $\begin{array}{l}\text { Compensated } \\
\text { Neu:ron/Gama Ray }\end{array}$ & Dresser Atlas & W7479Y & SurE. & 654 & $9 / 12 / 78$ \\
\hline Dual Laterolog & Dresser Atlas & w74792 & Surf. & 652 & $9 / 12 / 78$ \\
\hline $\begin{array}{l}\text { Digital Velocity } \\
\text { Conversion }\end{array}$ & $\begin{array}{l}\text { Seismic Reference } \\
\text { Service, Inc. } 4\end{array}$ & W7480Y & 0 & 647 & $10 / 5 / 78$ \\
\hline M : oro-Lat erol $\propto 9$ & Dresser Atlas & W7480W & Sur $€$. & 653 & $9 / 12: 75$ \\
\hline
\end{tabular}

Lor 1 ginal data is retained in Sandia WIPP Central File, Division 4542, Sandi a Laboratories, A.l buquerque, NM, 87185.

2orjer number for logs avallable through west Texas Electric Log Service. Inr. :E[SI], 105 West Wᄏ1l Avenue, Mldland, TX.

'Depths measured from ground surface; elevation officially 3212.44 ' above MSL.

${ }^{4}$ Selsmic Reference service, Inc., Tulsa, ok. 
Distcibution:

U.S. Department of Energy, Headquarters

OEE ice of Nuclear waste Management

Washington, DC 20545

Eugene F. Beckett, Project Coordinator (WIPP) (5)

Colin A. Heath, Director, Division of Waste Isolation

U.S. Department of Energy, Albuquerque Operations

P.O. Box 5400

Albuquerque, NM 87185

D. T. Schueler, Manager, WIPP Project office

G. Dennis, Director, Public Affairs Division

s. C. Taylor, CaTI Division

U.S. Department of Energy

Carlsbad WIPP Project office

Room 113, Federal Building

Carlsbad, NM 68220

U.S. Department of Energy

c/o Battelle OEfice of Nuclear

Waste I solation

$505 \mathrm{King}$ Avenue

Columbus, OH 43201

Jeff $O$. Neff

Battelie Memorial Institute

office of Nuclear Waste Isolation

$505 \mathrm{King}$ Avenue

Columbus, OH 43201

Neil Carter, General Manager (5)

restinghouse Electric Corporation

P. O. Box 40039

Albuguerque, NM 87196

R. C. Mairson (3)

Hobbs Public Library

509 N. Ship St.

l.obbs, NM 88248

Ms. Marcia Lewis, Librarian

Lokes h $C$ hat ur vedi

Department of Civil Engl neering

Box 3E

New Mexi $\infty$ State University

Las Cruces, NM 88003 


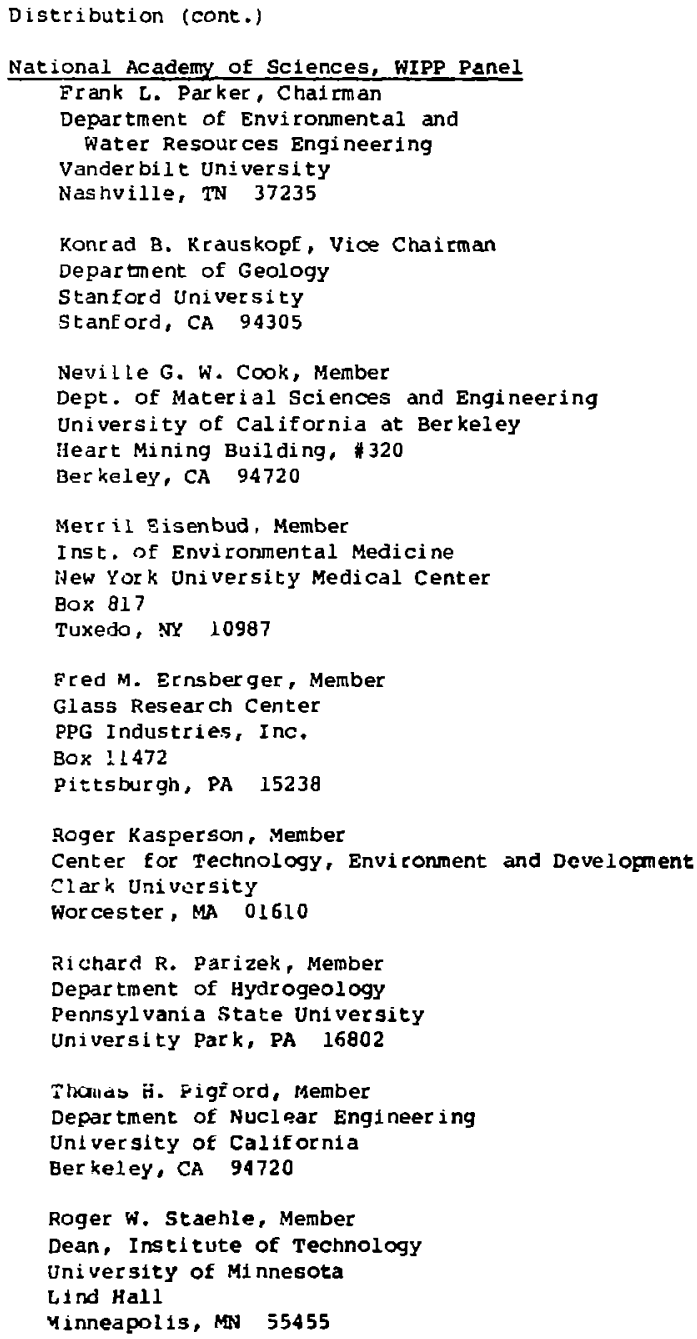




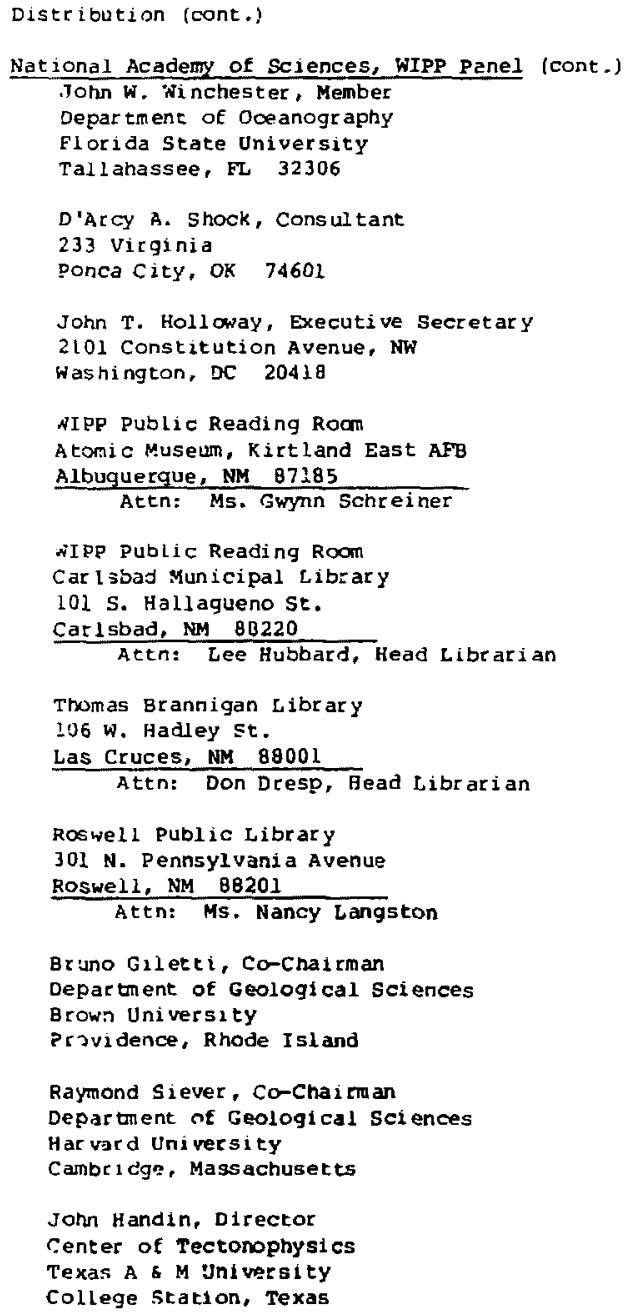




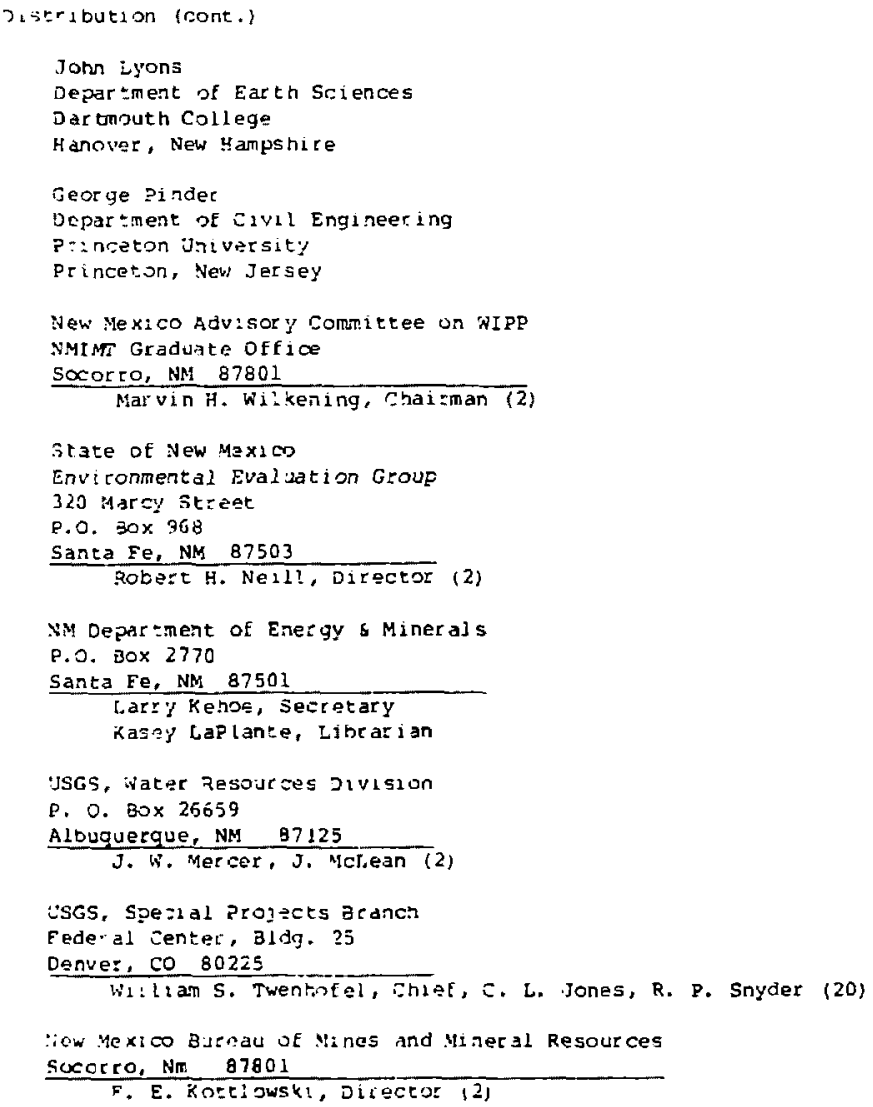




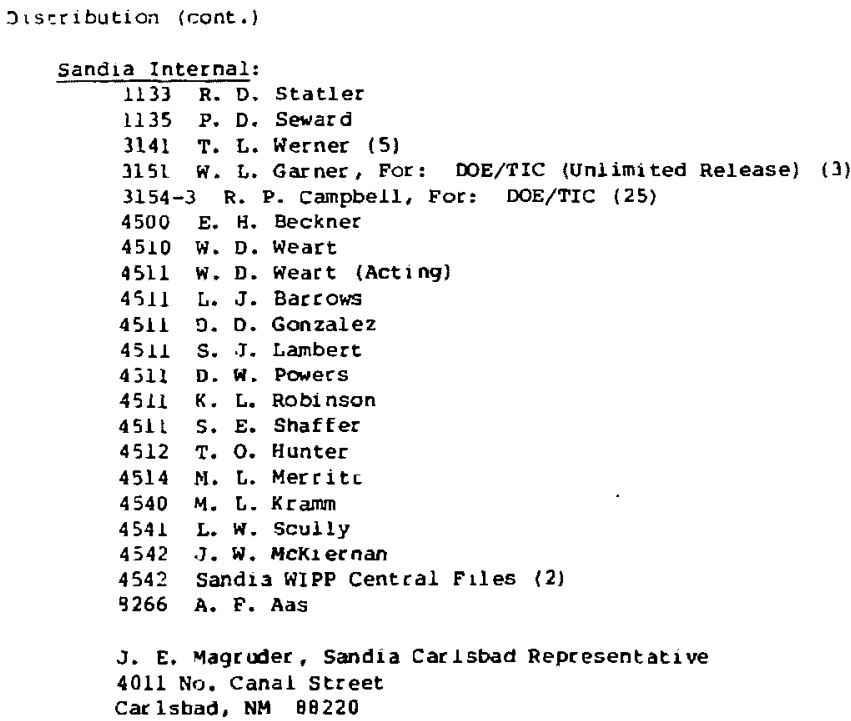

